ESAIM: COCV 27 (2021) 58

https://doi.org/10.1051/cocv/2021055
ESAIM: Control, Optimisation and Calculus of Variations

www.esaim-cocv.org

\title{
AN OPTIMAL CONTROL PROBLEM RELATED TO A 3D-CHEMOTAXIS-NAVIER-STOKES MODEL*,**
}

\author{
J. LÓPEZ-RÍOS AND ÉLDER J. VILlAMIZAR-ROA***
}

\begin{abstract}
In this paper, we study an optimal control problem associated to a 3D-chemotaxis-NavierStokes model. First we prove the existence of global weak solutions of the state equations with a linear reaction term on the chemical concentration equation, and an external source on the velocity equation, both acting as controls on the system. Second, we establish a regularity criterion to get global-in-time strong solutions. Finally, we prove the existence of an optimal solution, and we establish a first-order optimality condition.
\end{abstract}

Mathematics Subject Classification. 35Q35, 35K51, 49J20, 49K20, 76D55.

Received March 26, 2020. Accepted May 20, 2021.

\section{INTRODUCTION}

The chemotaxis phenomenon describes the movement of cells directed by the concentration gradient of a chemical substance in their environment. One of the most interesting phenomena in chemotaxis corresponds to the movement of cells toward the increasing concentration of a chemical signal which is consumed by cells themselves. Also, it has been observed that interactions between cells and the chemical signal with liquid environments play an important role in various biological processes; indeed, it was observed that when bacteria of the species Bacillus subtilis are suspended in water, some spatial patterns may spontaneously emerge from initially almost homogeneous distributions of bacteria [15, 49]. A mathematical model to describe this processes was proposed in [47]; it is given by the following system of Partial Differential Equations:

$$
\left\{\begin{array}{l}
n_{t}+u \cdot \nabla n=D_{n} \Delta n-\chi \nabla \cdot(n \nabla c), \\
c_{t}+u \cdot \nabla c=D_{c} \Delta c-\gamma c n \\
\rho\left(u_{t}+(u \cdot \nabla) u\right)=\eta \Delta u-\nabla \pi+n \nabla \Phi \\
\nabla \cdot u=0
\end{array}\right.
$$

where $n=n(x, t) \geq 0, c=c(x, t) \geq 0, \pi(x, t)$ and $u(x, t)$ denote respectively the cell density, the concentration of an attractive chemical signal, the hydrostatic pressure, and the velocity of the fluid at position $x \in \Omega \subseteq \mathbb{R}^{3}$

* J. López-Ríos was supported by MINCIENCIAS, convocatoria 848-2019, posdoc project Fondo Nacional de Financiamiento Para la Ciencia, la Tecnología y la Innovación "Francisco José de Caldas", number 80740-067-2020.

**E.J. Villamizar-Roa was supported by Vicerrectoría de Investigación y Extensión of Universidad Industrial de Santander, Project "Estudio teórico de problemas de quimiotaxis-haptotaxis en dominios no acotados", code C-2020-05.

Keywords and phrases: Optimal control problem, chemotaxis-fluid model, optimality conditions.

Universidad Industrial de Santander, Escuela de Matemáticas, A.A. 678, Bucaramanga, Colombia.

*** Corresponding author: jvillami@uis.edu.co 
and time $t \in(0, T], T>0$. This model describes the interaction between a type of cells (e.g., bacteria), and a chemical signal which is consumed with a rate proportional to the amount of organisms. The cells and chemical substances are transported by a viscous incompressible fluid under the influence of a force due to the aggregation of cells. The equation for the velocity field $u$ is described by the incompressible Navier-Stokes system with forcing term given by $n \nabla \Phi$, which represents the effects due to density variations caused by cell aggregation. The parameters $\chi, D_{n}, D_{c}, \rho, \gamma$ and $\eta$ are positive constants that represent the chemotactic coefficient, the cell diffusion coefficient, the chemical diffusion coefficient, the fluid density, the chemical consumed rate and the viscosity of fluid, respectively.

In this paper we are interested in the mathematical formulation and theoretical analysis of an optimal control problem for a chemotaxis process occurring inside a viscous and incompressible fluid. The control of the velocity, the proliferation of organisms and the concentration of chemicals in diverse environments have significant applications in science and biological processes. In fact, in several applications, the respective biological setting requires to control the proliferation and death of cells, for example, bacterial pattern formation $[48,55]$ or endothelial cell movement and growth in response to a chemical substance known as tumor angiogenesis factor (TAF), which have a significant role in the process of cancer cell invasion of neighboring tissue $[7,8,35]$. In this paper we propose the analysis of an optimal control problem given by the minimization of a general cost functional subject to constraints, where the state equations are given by the model (1.1). The controls act on the concentration and the velocity equations as follows:

$$
\left\{\begin{array}{l}
n_{t}+u \cdot \nabla n=D_{n} \Delta n-\chi \nabla \cdot(n \nabla c), \\
c_{t}+u \cdot \nabla c=D_{c} \Delta c-\gamma c n+g c \\
u_{t}+(u \cdot \nabla) u=\eta \Delta u-\nabla \pi+n \nabla \Phi+f \\
\nabla \cdot u=0
\end{array}\right.
$$

in $\Omega_{T}=\Omega \times(0, T)$, where $g$ and $f$ are given controls acting on the chemical concentration and the fluid velocity, respectively. Notice that in the region of $\Omega$ where $g \geq 0$ the control acts as a proliferation source of the chemical substance, and inversely, in the region of $\Omega$ where $g \leq 0$ the control acts as a degradation source of the chemical substance. This kind of control is commonly known as bilinear control. It is worthwhile to remark that, with this kind of bilinear control we are able to guarantee the non-negativity of the unknowns $n, c$ of (1.2); if we consider a distributed control, that is, if we take in the second equation (1.2) $g$ in place of $c g$, we have to assume that $g \geq 0$ in order to guarantee the non-negativity of $c$. System (1.2) is completed with the following initial and non-flux boundary data:

$$
\left\{\begin{array}{l}
{[n(0), c(0), u(0)]=\left[n_{0}, c_{0}, u_{0}\right], n_{0}, c_{0} \geq 0, x \in \Omega,} \\
\frac{\partial n}{\partial \nu}=\frac{\partial c}{\partial \nu}=0, \quad u=0, \quad \text { on } \partial \Omega, \quad t \in(0, T)
\end{array}\right.
$$

where $\nu$ denotes the outward unit normal vector to $\partial \Omega$. Thus, the aim of this paper is to analyze an optimal control problem in such a way that any admissible state is a strong solution of (1.2)-(1.3). Since the existence of global in time strong solutions of (1.2)-(1.3) is an open question, we have to choose a suitable cost functional $J([n, c, u])$, considering in particular the $L^{20 / 7}\left(L^{20 / 7}\right)$ norm for the density. Thus, if $[n, c, u]$ is a weak solution of $(1.2)-(1.3)$ in $(0, T)$ such that $J([n, c, u])<\infty$, then $n \in L^{20 / 7}\left(L^{20 / 7}\right)$ and as consequence of a regularity criterion, we will show that $[n, c, u]$ is a strong solution of $(1.2)-(1.3)$ in $(0, T)$. Precisely, we wish to minimize the functional $J: \mathcal{A} \times \mathcal{B} \rightarrow \mathbb{R}$ defined by

$$
\begin{aligned}
J([f, g])= & \frac{7}{20} \int_{0}^{T}\|n(t)-\bar{n}(t)\|_{L^{20 / 7}}^{20 / 7} \mathrm{~d} t+\frac{1}{2} \int_{0}^{T}\|c(t)-\bar{c}(t)\|_{L^{2}}^{2} \mathrm{~d} t \\
& +\frac{1}{8} \int_{0}^{T}\|u(t)-\bar{u}(t)\|_{L^{4}}^{8} \mathrm{~d} t+\frac{\alpha_{1}}{2} \int_{0}^{T}\|f(t, x)\|_{L^{2}}^{2} \mathrm{~d} t \\
& +\frac{\alpha_{2}}{4} \int_{0}^{T}\|g(t, x)\|_{L^{4}}^{4} \mathrm{~d} t
\end{aligned}
$$


where $\mathcal{A} \times \mathcal{B}$ denotes a suitable set of admissible controls and the functions $n, c, u$ are subject to satisfy the state system (1.2)-(1.3). The functions $\bar{n}, \bar{c}, \bar{u}$ are given and denote the desired states for density, concentration and velocity, and the parameters $\alpha_{1}, \alpha_{2}>0$ stand for the cost coefficients of the control. The exact mathematical formulation will be given in Section 3. Without loss of generality, from now on we will assume $D_{n}=D_{c}=\eta=1$. We will prove the solvability of the optimal control problem and state the first-order optimality conditions.

In order to study the optimal control problem described above, firstly we need to clarify the existence and uniqueness of a solution for (1.2)-(1.3). The mathematical understanding of the existence and uniqueness of a solution for (1.2)-(1.3) (with $f, g=0)$ is quite challenging, due to the coupling between the Navier-Stokes equations and the chemotaxis system. Indeed, as is well known, the 3D Navier-Stokes system has not a satisfactory existence theory; it is known the existence of global weak solutions $u \in L^{2}\left(0, T ; H_{0}^{1}(\Omega)\right) \cap C_{w}\left([0, T] ; L^{2}(\Omega)\right)$, for initial data in $L^{2}(\Omega)$. However, in contrast to the $2 \mathrm{D}$ case, the uniqueness is still an open problem. On the other hand, for the chemotaxis subsystem of (1.2), obtained upon neglecting the fluid interactions, contrary to the 2D case, only certain weak solutions are known to exist globally ( $c f$. [44]). The full chemotaxis-fluid model (1.2)(1.3) (with $f, g=0$ ) has been analyzed in $[4,16,28,45,49-51,53,58]$. In [49], the existence of global classical solutions was proved in two-dimensional bounded convex domains. The results of [49] were extended to nonconvex domains in [29]. Results of convergence of classical solutions to the corresponding stationary model were analyzed in $[52,57]$. In [49] was also proved the existence of global weak solutions in bounded three-dimensional convex domains, considering the Stokes system in place of Navier-Stokes equation, that is, neglecting the nonlinear term $(u \cdot \nabla) u$ in the fluid equation. The existence of global weak solutions for the full three-dimensional bounded convex domains Chemotaxis Navier-Stokes model was obtained in [51]. There, the author also proved that any eventual energy solution becomes smooth after some waiting time. The existence of weak solutions for (1.2)-(1.3) given in [51] (with $f, g=0$ ) was obtained as the limit of smooth solutions to suitably regularized problems, where appropriate compactness properties are derived on the basis of a priori estimates gained from an energy-type inequality, which combines the standard $L^{2}$ dissipation property of the fluid evolution with a quasi-dissipative structure associated with the chemotaxis subsystem. The existence of classical solutions for the regularized problems are obtained by applying a fixed point procedure to ensure local existence and uniqueness of smooth solutions, and deriving a suitable extension criterion for such solutions, by using a priori estimates. A further step toward a qualitative understanding of the chemotaxis-fluid interaction described by (1.2), in terms of the possible effects of the chemotaxis-driven forcing on the motion, and the latter on the distribution of cells was given in [53]. The author shows that any mutual influence will in fact dissappear asymptotically, in that the large-time behavior of solutions is essentially governed by the decoupled chemotaxis only and NavierStokes subsystems on neglecting the components $u$ and $[n, c]$, respectively. Variants of (1.2)-(1.3) including nonlinear cell diffusion, that is, replacing $\Delta n$ by the porous medium-type diffusion term $\Delta^{m} n$ for $m>1$, and assuming that $\chi$ is a chemotactic sensitivity tensor, that is, replacing $\chi \nabla \cdot(n \nabla c)$ by $\nabla \cdot(n S(n, c, x) \nabla c)$, where $S(n, c, x)=\left(s_{i, j}\right)_{N \times N}$ is a matrix-valued function, have been analyzed in $[4,16,28,45,50,54,58]$ and some references therein. In these cases, convenient boundary conditions are assumed. System (1.2)-(1.3) with logistic source $\left(f(n)=\varsigma n-\mu n^{2}\right.$ with $\left.\mu>0\right)$, has been analyzed in [34]. Considering bounded domains of $\mathbb{R}^{3}$, in [34] the author analyzed the existence of weak solutions and proved that the weak solutions become smooth after some waiting time; moreover, convergence to the steady state $(\varsigma / \mu, 0,0)$ was also established. In [3], it was considered an exchange of oxygen between the fluid and its environment, which leads to different boundary conditions to (1.2); then, by requiring sufficiently smooth initial data, it was proved the existence of a unique global classical solution for $N=2$, as well as the existence of a global weak solution for $N=3$. Concerning smooth bounded domains and initial data in $L^{p}$-spaces, results about local and global-in-time existence of solutions were obtained in [17]. A numerical analysis based on the Finite Element method including error estimates and convergence towards regular solutions were performed in [19]. On the other hand, in the whole space $\mathbb{R}^{N}, N=2,3$, the local and global-in-time existence of solutions for (1.2) has been studied in [11-13, 30, 56], and some references therein. In particular, a class of small global solutions in weak- $L^{p}$ spaces including double attraction nonlinear terms in the density equation was obtained in [30]. In [56] the local well-posedness for initial data in the nonhomogeneous Besov spaces class $B_{p, r}^{s} \times B_{p, r}^{s+1} \times B_{p, r}^{s+1}$ where $1<p<\infty, 1 \leq r \leq \infty$ and $s>3 / p+1$ was analyzed. In [13] the local-in-time existence of solutions for large initial data, as well as global-in-time existence for small initial data 
and some smallness condition on the gravitational term, were obtained in critical homogeneous Besov spaces. Later, an extension criterion for local-in-time solutions was proved in [11]. In [12] the global-in-time existence of solutions for small initial data in the critical triple of homogeneous Besov spaces $\dot{B}_{p, 1}^{-2+3 / p} \times \dot{B}_{p, 1}^{3 / p} \times \dot{B}_{p, 1}^{-1+3 / p}$ with $1 \leq p<3$ was proved. More recently, in $[18,21]$ results on existence and asymptotic behavior of small global solutions in Besov-Morrey spaces were obtained.

However, the derivation of the energy-like estimates when $f, g \neq 0$ in (1.2) do not follow directly due principally to the term $g c$ in the derivation of energy-type estimates and uniform estimates for the chemical concentration, by considering a nonregular control $g \in L^{p}\left(L^{p}\right)$ for some $p>1$ (see details in Sect. 3). Therefore, the first aim of this paper is to prove the existence of weak solutions for (1.2). In comparison with the results of [51], our definition of weak solution establishes that the equation for the chemoattractant is satisfied a.e. in $\Omega \times(0, T)$, that is, our weak solution is weaker than the one in [51]. Moreover, following [29, 34], we do not assume the convexity condition on $\Omega$ as required in [49, 51]. In order to prove the existence of weak solutions of the control system we consider a family of regular solutions to a suitable regularized problem, which is a little different from that considered in [51]. Indeed, we introduce a decoupling through an auxiliary elliptic problem which allows to gain regularity for the chemical equation as well as to obtain an energy-type inequality after testing and combining conveniently the density equation and the concentration equation (see details in Sect. 3.1).

In connection to the existence of weak solutions, it is worthwhile to remark that the uniqueness of weak solutions in 3D is an open problem; consequently, due to the lack of uniqueness of weak solutions nor the existence of strong solutions, establishing optimality conditions when analyzing control problems becomes nontrivial. As a consequence, most of the studies devoted to the control problems in fluid mechanics and related models assume that $\Omega \subset \mathbb{R}^{2}(c f .[2,5])$. In order to overcome this difficulty, the second aim of this paper is to introduce a regularity criterion which allow us to get a unique strong solution ( $c f$. Sect. 4). This regularity criterion is motivated by the corresponding one for the Navier-Stokes system $[2,5]$.

On the other hand, having decided what kind of solutions of the Chemotaxis-Navier-Stokes equations (1.2)(1.3) are going to be considered, the third aim of this paper is to analyze an optimal control problem. Here we recall that in past years, significant progress has been made in mathematical analysis and numerics of optimal control problems for viscous flows described by the Navier-Stokes equations and related models (see for instance, $[2,5,23,36]$ and references therein). However, from the optimal control point of view, the literature related is scarce, and most of the results are devoted to the control theory governed by chemotaxis models without fluids (cf. $[14,22,24-26,40])$. In these references, the authors proved the existence of optimal controls and derived an optimality system. However, as far as we know, optimal control problems for evolutive attractive chemotaxisNavier-Stokes models have not been studied previously. In [39] the authors analyze an optimal distributed control problem where the state equations are given by a stationary chemotaxis model coupled with the Navier-Stokes equations. The control was given through a distributed force and a coefficient of chemotactic sensitivity, leading the chemical concentration, the cell density, and the velocity field towards a given target concentration, density and velocity, respectively. In spite of, we observe that in $[9,10]$ the authors obtained some results related to the controllability for the nonstationary Keller-Segel system and the nonstationary chemotaxis-fluid model with consumption of chemoattractant substance associated to a chemotaxis system, based on Carleman estimates for the solutions of the adjoint system. We formulate the control problem in such a way that any optimal state is a strong solution, and prove the existence of an optimal solution assuming controls on the concentration and velocity, such that the associated strong solution exists.

The rest of the paper is organized as follows: in Section 2 we introduce some basic notations and preliminary results. In Section 3, we give the definition of weak solutions for system (1.2)-(1.3) and prove that they exist indeed. In Section 4, we establish a regularity criterion under which weak solutions of (1.2)-(1.3) are also strong solutions. In Section 5, we analyze an optimal control problem related to the strong solutions of (1.2)-(1.3); we prove the existence of an optimal solution and derive first-order optimality conditions. 


\section{NotATIONS AND PRELIMINARIES}

In this section we establish some basic notations, and give some preliminary results to be used later. Hereafter, $\Omega$ is a bounded domain of $\mathbb{R}^{3}$ with boundary of class $C^{2,1}$. The reason to pick this particular regularity on the domain is the $C^{2}$-parabolic and $C^{1,1}$-elliptic regularities for the Laplace problem on a domain (see Thm. 2.1 and system (3.3)). We use the Sobolev space $W^{k, p}(\Omega)$ and $L^{p}(\Omega), k \in \mathbb{R}, 1 \leq p \leq \infty$, with norms $\|\cdot\|_{W^{k, p}}$ and $\|\cdot\|_{L^{p}}$ respectively. When $p=2$, we write $H^{k}(\Omega):=W^{k, 2}(\Omega)$ and its norm will be denoted by $\|\cdot\|_{H^{k}}$. The inner product in $L^{2}(\Omega)$ will be represented by $(\cdot, \cdot)$. We denote by $W_{0}^{1,2}(\Omega)$ the closure of $C_{0}^{\infty}(\Omega)$ in the norm $\|\cdot\|_{W^{1,2}}$. We also consider the free divergence spaces $V=\left\{u \in\left(W_{0}^{1,2}(\Omega)\right)^{3}: \operatorname{div} u=0\right.$ in $\left.\Omega\right\}$, and $\left(L_{\sigma}^{2}(\Omega)\right)^{3}$ given by the closure of $\left\{u \in C_{0}^{\infty}(\Omega)^{3}\right.$, div $\left.u=0\right\}$ in $L^{2}(\Omega)$. We also recall the Leray projector $P:\left(L^{2}(\Omega)\right)^{3} \rightarrow\left(L_{\sigma}^{2}(\Omega)\right)^{3}$, and denote by $A:=-P \Delta$ the Stokes operator with domain $D(A)=\left(H^{2}(\Omega)\right)^{3} \cap V$.

Along this work, we are going to fix a time $0<T<\infty$ but arbitrary. In this sense, we introduce the following notation. For $Y$ being a Banach space, $L^{p}(Y):=L^{p}(0, T ; Y), 1 \leq p \leq \infty$, denotes the space of Bochner integrable functions defined on the interval $[0, T]$ with values in $Y$, endowed with the usual norm $\|\cdot\|_{L^{p}(Y)}$. We also consider the space $C(Y):=C([0, T] ; Y)$ of continuous functions from $[0, T]$ into $Y$, with norm $\|\cdot\|_{C(Y)}$.

The topological dual of a Banach space $Y$ will be denoted by $Y^{\prime}$, and the duality product by $\langle\cdot, \cdot\rangle_{Y^{\prime}}$ or simply $\langle\cdot, \cdot\rangle$ when there is no confusion. For simplicity in the notation, we use $u$ for both scalar and vector valued functions. Also, the letter $C$ will denote a general positive constant which may change from line to line or even within the same line.

In order to analyze properties of existence and regularity of solutions (1.2)-(1.3), frequently, we will use the following parabolic regularity result.

Theorem 2.1 ([20], Thm. 10.22). Let $\Omega$ be a bounded domain with boundary $\partial \Omega$ of class $C^{2}, 1<p, q<\infty$. Suppose that $f \in L^{p}\left(L^{q}\right), u_{0} \in Z_{p, q}=\left\{L^{q}(\Omega) ; \mathrm{d}\left(\Delta_{\mathcal{N}}\right)\right\}_{1-1 / p, p}, \mathrm{~d}\left(\Delta_{\mathcal{N}}\right)=\left\{v \in W^{2, q}(\Omega): \frac{\partial u}{\partial \nu}=0\right.$ on $\left.\Omega\right\}$, where $\{\cdot ; \cdot\} .$, denotes the real interpolation space. Then the parabolic problem

$$
\begin{cases}\partial_{t} u-\Delta u=f, & \text { in }(0, T) \times \Omega, \\ u(0, \cdot)=u_{0}, & \text { in } \Omega, \\ \frac{\partial u}{\partial \nu}=0, & \text { on }(0, T) \times \partial \Omega,\end{cases}
$$

admits a unique solution $u$ such that

$$
u \in C\left(Z_{p, q}\right) \cap L^{p}\left(W^{2, q}\right), \quad \partial_{t} u \in L^{p}\left(L^{q}\right) .
$$

Moreover, there exists a positive constant $C:=C(p, q, \Omega, T)$ such that

$$
\|u(t)\|_{C\left(Z_{p, q}\right)}+\left\|\partial_{t} u\right\|_{L^{p}\left(L^{q}\right)}+\|\Delta u\|_{L^{p}\left(L^{q}\right)} \leq C\left(\|f\|_{L^{p}\left(L^{q}\right)}+\left\|u_{0}\right\|_{Z_{p, q}}\right) .
$$

If $p=q$, it holds that

$$
Z_{p, p}=\widehat{W}^{2-2 / p, p}=\left\{\begin{array}{l}
W^{2-2 / p, p}(\Omega), \text { if } p<3 \\
\left\{u \in W^{2-2 / p, p}(\Omega): \frac{\partial u}{\partial \nu}=0 \text { on } \partial \Omega\right\}, \text { if } p>3 .
\end{array}\right.
$$

We will denote by $\mathcal{X}_{p}$ the space

$$
\mathcal{X}_{p}=\left\{u \in C\left(Z_{p, p}\right) \cap L^{p}\left(W^{2, p}\right): \partial_{t} u \in L^{p}\left(L^{p}\right)\right\}
$$




\section{EXISTENCE OF WEAK SOLUTIONS}

In this section we prove the existence of global weak solutions for the control system (1.2)-(1.3). We obtain the existence of weak solutions as the limit of smooth solutions to suitable regularized problems, where appropriate compactness properties are derived from a priori estimates gained from an energy-type inequality. We start by establishing the notion of weak solutions for (1.2)-(1.3).

Definition 3.1. (Weak solution) Let $0<T<\infty$, and $g \in L^{5 / 2}\left(L^{5 / 2}\right), f \in L^{2}\left(L^{2}\right), \nabla \Phi \in L^{\infty}(\Omega), n_{0} \in L^{2}(\Omega)$, $c_{0} \in W^{1, q}(\Omega), q>3, u_{0} \in L^{2}(\Omega), n_{0} \geq 0, c_{0} \geq 0$ in $\Omega$. A weak solution of (1.2)-(1.3) is a triple $[n, c, u]$ of functions such that

$$
\begin{array}{r}
n \in L^{5 / 3}\left(L^{5 / 3}\right) \cap L^{5 / 4}\left(W^{1,5 / 4}\right), \partial_{t} n \in L^{10 / 9}\left(\left(W^{1,10}\right)^{\prime}\right), \\
c \in L^{\infty}\left(H^{1}\right) \cap L^{2}\left(H^{2}\right), \partial_{t} c \in L^{10 / 7}\left(L^{10 / 7}\right), \\
u \in L^{2}(V) \cap L^{\infty}\left(L^{2}\right), \partial_{t} u \in L^{5 / 3}\left(\left(W^{1,5 / 2}\right)^{\prime}\right),
\end{array}
$$

verifying

$$
\begin{array}{r}
\int_{0}^{T}\left\langle\partial_{t} n, \varphi\right\rangle+\int_{0}^{T} \int_{\Omega} \nabla n \cdot \nabla \varphi-\int_{0}^{T} \int_{\Omega} n u \cdot \nabla \varphi-\chi \int_{0}^{T} \int_{\Omega} n \nabla c \cdot \nabla \varphi=0 \\
\int_{0}^{T}\left\langle\partial_{t} u, \psi\right\rangle+\int_{0}^{T} \int_{\Omega} \nabla u \cdot \nabla \psi-\int_{0}^{T} \int_{\Omega} u \otimes u \cdot \nabla \psi=\int_{0}^{T} \int_{\Omega} n \nabla \Phi \cdot \psi+\int_{0}^{T} \int_{\Omega} f \cdot \psi
\end{array}
$$

for all $\varphi \in L^{10 / 3}\left(W^{1,10 / 3}\right)$, and $\psi \in L^{5 / 2}\left(W^{1,5 / 2}\right)$, and the equation $(1.2)_{2}$ holds a.e $(x, t)$ in $\Omega \times(0, T)$.

In order to obtain the existence of weak solutions, we consider the following assumption on the data:

$$
\left\{\begin{array}{l}
g \in L^{5 / 2}\left(L^{5 / 2}\right), f \in L^{2}\left(L^{2}\right), \nabla \Phi \in L^{\infty}(\Omega), \\
n_{0} \in L^{2}(\Omega), c_{0} \in W^{1, q}(\Omega), q>3, u_{0} \in L_{\sigma}^{2}(\Omega), \\
n_{0}(x)>0, c_{0}(x)>0 .
\end{array}\right.
$$

We prove the following theorem of global existence of weak solutions.

Theorem 3.2. (Existence of weak solution) Assume that $g, f, \nabla \Phi$, and the initial data $\left[n_{0}, c_{0}, u_{0}\right]$ satisfy (3.1). Then there exists a weak solution of system (1.2)-(1.3), in the sense of Definition 3.1.

Remark 3.3. In comparison with the definition of weak solution considered in [51], in Definition 3.1, the equation for the concentration of chemical is satisfied a.e in $\Omega \times(0, T)$.

\subsection{Approximated solutions}

The existence of weak solutions of (1.2)-(1.3) is obtained through as the limit of a sequence of strong solutions of the following approximated problems: given $\epsilon>0$, find $\left[n_{\epsilon}, w_{\epsilon}, u_{\epsilon}, \pi_{\epsilon}\right]$ such that:

$$
\left\{\begin{array}{l}
n_{\epsilon t}+u_{\epsilon} \cdot \nabla n_{\epsilon}=\Delta n_{\epsilon}-\chi \nabla \cdot\left(\frac{n_{\epsilon}^{+}}{1+\epsilon n_{\epsilon}^{+}} \nabla c_{\epsilon}\right) \quad \text { in } \Omega \times(0, T), \\
w_{\epsilon t}+u_{\epsilon} \cdot \nabla w_{\epsilon}=\Delta w_{\epsilon}-\gamma w_{\epsilon}^{+} \frac{1}{\epsilon} \ln \left(1+\epsilon n_{\epsilon}^{+}\right)+g w_{\epsilon}^{+} \quad \text { in } \Omega \times(0, T), \\
u_{\epsilon t}+\left(Y_{\epsilon} u_{\epsilon} \cdot \nabla\right) u_{\epsilon}=\Delta u_{\epsilon}-\nabla \pi_{\epsilon}+n_{\epsilon} \nabla \Phi+f \quad \text { in } \Omega \times(0, T), \\
\nabla \cdot u_{\epsilon}=0 \quad \text { in } \Omega \times(0, T), \\
{\left[n_{\epsilon}(0), w_{\epsilon}(0), u_{\epsilon}(0)\right]=\left[n_{0, \epsilon}, w_{0, \epsilon}, u_{0, \epsilon}\right] \quad \text { in } \Omega,} \\
\frac{\partial n_{\epsilon}(x, t)}{\partial \nu}=\frac{\partial w_{\epsilon}(x, t)}{\partial \nu}=0, \quad u_{\epsilon}(x, t)=0 \quad \text { on } \partial \Omega \times(0, T),
\end{array}\right.
$$


where $Y_{\epsilon}=(1+\epsilon A)^{-1}$ is the Yosida approximation [43], and $c_{\epsilon}$ is the unique solution of the elliptic problem

$$
\left\{\begin{array}{l}
c_{\epsilon}-\epsilon \Delta c_{\epsilon}=w_{\epsilon} \text { in } \Omega \\
\frac{\partial c_{\epsilon}(x, t)}{\partial \nu}=0 \text { on } \partial \Omega
\end{array}\right.
$$

In (3.2), $n_{\epsilon}^{+}=\max \left\{n_{\epsilon}, 0\right\} \geq 0, w_{\epsilon}^{+}=\max \left\{w_{\epsilon}, 0\right\} \geq 0$, and for all $\epsilon \in(0,1), n_{0, \epsilon}, c_{0, \epsilon}, u_{0, \epsilon}$ are initial data satisfying:

$$
\left\{\begin{array}{l}
n_{0, \epsilon} \in W^{4 / 5,5 / 3}(\Omega), n_{0, \epsilon}>0, \int_{\Omega} n_{0, \epsilon}=\int_{\Omega} n_{0}, n_{0, \epsilon} \rightarrow n_{0} \text { in } L^{2}(\Omega), \text { as } \epsilon \rightarrow 0 \\
w_{\epsilon, 0} \geq 0, w_{0, \epsilon}=c_{0, \epsilon}-\epsilon \Delta c_{0, \epsilon} \in W^{1, q}(\Omega), q>3 \\
c_{0, \epsilon} \geq 0, c_{0, \epsilon} \rightarrow c_{0} \text { in } H^{1}(\Omega), \text { as } \epsilon \rightarrow 0 \\
w_{0, \epsilon} \rightarrow c_{0} \text { in } W^{1, q}(\Omega), \text { as } \epsilon \rightarrow 0 \\
u_{0, \epsilon} \in V, u_{0, \epsilon} \rightarrow u_{0} \text { in } L^{2}(\Omega), \text { as } \epsilon \rightarrow 0
\end{array}\right.
$$

Remark 3.4. Approximation (3.2) differs from that considered in [51], in the following aspects: first, we decouple the cross-diffusion term by introducing the elliptic problem (3.3). This allows enhancing regularity for the new variable $c_{\epsilon}$, which, in particular, enables the use of the Leray-Schauder fixed point theorem. This procedure is not required in [51] due to the use of the semigroup theory to get existence of local strong solutions. However, in our case, we were able to consider a different class of initial data providing a new class of global weak solutions, weaker than those in Definition 2.1 of [51]. Second, our approximation includes some non-negative terms at the right hand side of (3.2), in order to guarantee the non negativeness of $n_{\epsilon}, w_{\epsilon}$. Approximation in the $w_{\epsilon}$-equation allows to cancel the cross-diffusion in the $n_{\epsilon}$-equation with the consumption term in the $w_{\epsilon}$-equation. The Yosida approximation for the $u_{\epsilon}$-equation was the regularization for the velocity used in [51].

The existence of strong solutions for (3.2)-(3.4) is given in the next proposition.

Proposition 3.5. For each $\epsilon>0$, there exist unique functions $n_{\epsilon} \in \mathcal{X}_{5 / 3}, w_{\epsilon} \in \mathcal{X}_{5 / 3}, u_{\epsilon} \in \mathcal{X}_{2}$, with $n_{\epsilon} \geq 0$, $w_{\epsilon} \geq 0$, which, together with some $\pi_{\epsilon} \in L^{2}\left(H^{1}\right)$ solve the system (3.2) pointwisely a.e. $(x, t) \in \Omega \times(0, T)$.

Proof. The proof will be carried out through the Leray-Schauder fixed point theorem. For that let us define the Banach space $X=L^{\infty}\left(L^{2}\right) \cap L^{2}\left(H^{1}\right)$ and the operator $\Gamma: X \times X \rightarrow X \times X$ by $\Gamma\left(\left[\bar{n}_{\epsilon}, \bar{w}_{\epsilon}\right]\right)=\left[n_{\epsilon}, w_{\epsilon}\right]$, where $n_{\epsilon}, w_{\epsilon}$ are the two first components of the solution $\left[n_{\epsilon}, w_{\epsilon}, u_{\epsilon}, \pi_{\epsilon}\right]$ for the following system:

$$
\left\{\begin{array}{l}
n_{\epsilon t}-\Delta n_{\epsilon}=-u_{\epsilon} \cdot \nabla \bar{n}_{\epsilon}-\chi \nabla \cdot\left(\frac{\bar{n}_{\epsilon}^{+}}{1+\epsilon \bar{n}_{\epsilon}^{+}} \nabla \bar{c}_{\epsilon}\right) \text { in } \Omega \times(0, T), \\
w_{\epsilon t}-\Delta w_{\epsilon}=-u_{\epsilon} \cdot \nabla \bar{w}_{\epsilon}-\gamma \bar{w}_{\epsilon}^{+} \frac{1}{\epsilon} \ln \left(1+\epsilon \bar{n}_{\epsilon}^{+}\right)+g \bar{w}_{\epsilon}^{+} \text {in } \Omega \times(0, T), \\
u_{\epsilon t}-\Delta u_{\epsilon}+\left(Y_{\epsilon} u_{\epsilon} \cdot \nabla\right) u_{\epsilon}+\nabla \pi_{\epsilon}=\bar{n}_{\epsilon} \nabla \Phi+f \text { in } \Omega \times(0, T), \\
\nabla \cdot u_{\epsilon}=0 \text { in } \Omega \times(0, T), \\
{\left[n_{\epsilon}(0), w_{\epsilon}(0), u_{\epsilon}(0)\right]=\left[n_{0, \epsilon}, w_{0, \epsilon}, u_{0, \epsilon}\right] \quad \text { in } \Omega,} \\
\frac{\partial n_{\epsilon}(x, t)}{\partial \nu}=\frac{\partial w_{\epsilon}(x, t)}{\partial \nu}=0, \quad u_{\epsilon}(x, t)=0 \quad \text { on } \partial \Omega \times(0, T),
\end{array}\right.
$$

with $\bar{c}_{\epsilon}$ being the unique solution of (3.3) and right hand side $\bar{w}_{\epsilon}$ (note that the first and second equations are decoupled between them in system (3.5)). We divide the proof in four steps.

Step one. $\Gamma: X \times X \rightarrow X \times X$ is well defined.

Let $\left[\bar{n}_{\epsilon}, \bar{w}_{\epsilon}\right] \in X \times X$. Following [43], Chapter V, Section 2.3, since $\bar{n}_{\epsilon} \in X$, there exists a unique solution $u_{\epsilon} \in \mathcal{X}_{2} \hookrightarrow L^{10}\left(L^{10}\right)$ of $(3.5)_{3}-(3.5)_{4}$ with initial data $u_{0, \epsilon}$. Also, since $\bar{w}_{\epsilon} \in X$ and recalling that $\partial \Omega \in C^{1,1}$, from the elliptic regularity applied to the problem (3.3) (cf. [27], Thms. 2.4.2.7 and 2.5.1.1), there exists a unique 
solution $\bar{c}_{\epsilon} \in L^{\infty}\left(H^{2}\right) \cap L^{2}\left(H^{3}\right)$. Then $\nabla \bar{c}_{\epsilon} \in L^{\infty}\left(H^{1}\right) \cap L^{2}\left(H^{2}\right) \hookrightarrow L^{10}\left(L^{10}\right)$. Since $\nabla \bar{n}_{\epsilon}^{+} \in L^{2}\left(L^{2}\right)$ and

$$
\chi \nabla \cdot\left(\frac{\bar{n}_{\epsilon}^{+}}{1+\epsilon \bar{n}_{\epsilon}^{+}} \nabla \bar{c}_{\epsilon}\right)=\chi \frac{\bar{n}_{\epsilon}^{+}}{1+\epsilon \bar{n}_{\epsilon}^{+}} \Delta \bar{c}_{\epsilon}+\chi \nabla\left(\frac{\bar{n}_{\epsilon}^{+}}{1+\epsilon \bar{n}_{\epsilon}^{+}}\right) \cdot \nabla \bar{c}_{\epsilon}
$$

by the Hölder inequality we get

$$
\chi \nabla \cdot\left(\frac{\bar{n}_{\epsilon}^{+}}{1+\epsilon \bar{n}_{\epsilon}^{+}} \nabla \bar{c}_{\epsilon}\right) \in L^{5 / 3}\left(L^{5 / 3}\right)
$$

Moreover, since $u_{\epsilon} \in L^{\infty}\left(H^{1}\right) \cap L^{2}\left(H^{2}\right) \hookrightarrow L^{10}\left(L^{10}\right)$ we have $u_{\epsilon} \cdot \nabla \bar{n}_{\epsilon}, u_{\epsilon} \cdot \nabla \bar{w}_{\epsilon} \in L^{5 / 3}\left(L^{5 / 3}\right)$. Therefore the right hand sides of $(3.5)_{1}$ and $(3.5)_{2}$ are in $L^{5 / 3}\left(L^{5 / 3}\right)$ and thus, from Theorem 2.1, there exist unique $n_{\epsilon}, w_{\epsilon} \in \mathcal{X}_{5 / 3}$ such that

$$
\begin{aligned}
\left\|n_{\epsilon}\right\|_{\mathcal{X}_{5 / 3}} & \leq C\left(\left\|n_{0, \epsilon}\right\|_{W^{4 / 5,5 / 3}},\left\|u_{\epsilon}\right\|\left\|_{\mathcal{X}_{2}},\right\| \bar{n}_{\epsilon}\left\|_{X},\right\| \bar{w}_{\epsilon} \|_{X}\right), \\
\left\|w_{\epsilon}\right\|_{\mathcal{X}_{5 / 3}} & \leq C\left(\left\|c_{0, \epsilon}-\epsilon \Delta c_{0, \epsilon}\right\|_{W^{4 / 5,5 / 3}},\left\|u_{\epsilon}\right\|_{\mathcal{X}_{2}},\left\|\bar{n}_{\epsilon}\right\|_{X},\left\|\bar{w}_{\epsilon}\right\|_{X}\right) .
\end{aligned}
$$

Since $\mathcal{X}_{5 / 3} \hookrightarrow L^{2}\left(H^{3 / 2}\right) \cap L^{\infty}\left(L^{2}\right) \hookrightarrow X$, we conclude $\Gamma$ is well defined.

Step two. $\Gamma: X \times X \rightarrow X \times X$ is compact.

First notice that from (A.1) in the appendix, $\mathcal{X}_{5 / 3} \hookrightarrow L^{\infty}\left(H^{1 / 2}\right) \cap L^{5 / 3}\left(H^{17 / 10}\right)$. Thus, by Lemma A.3, $\mathcal{X}_{5 / 3} \hookrightarrow L^{2}\left(H^{3 / 2}\right) \cap L^{\infty}\left(H^{1 / 2}\right)$ and $H^{3 / 2} \stackrel{c}{\hookrightarrow} H^{1} \hookrightarrow L^{5 / 3}, H^{1 / 2} \stackrel{c}{\hookrightarrow} L^{2} \hookrightarrow L^{5 / 3}$, where $\stackrel{c}{\hookrightarrow}$ denotes the compact embedding. Thus, by the Aubin-Lions compactness theorem [31], and the Simon compactness result [41], the embedding of $\mathcal{X}_{5 / 3} \subset\left\{f \in L^{2}\left(H^{3 / 2}\right) \cap L^{\infty}\left(H^{1 / 2}\right): \partial_{t} u \in L^{5 / 3}\left(L^{5 / 3}\right)\right\}$ into $X$ is compact.

Step three. The fixed points of $\alpha \Gamma, \alpha \in[0,1]$, are bounded in $X \times X$.

We assume $\alpha \in(0,1]$ (the case $\alpha=0$ is obvious). If $\left[n_{\epsilon}, w_{\epsilon}\right]$ is a fixed point of $\alpha \Gamma$, then $\left[n_{\epsilon}, w_{\epsilon}\right]=\alpha \Gamma\left[n_{\epsilon}, w_{\epsilon}\right]$, which means that $\left[n_{\epsilon}, w_{\epsilon}\right]$ satisfies

$$
\left\{\begin{array}{l}
n_{\epsilon t}-\Delta n_{\epsilon}=-u_{\epsilon} \cdot \nabla n_{\epsilon}-\alpha \chi \nabla \cdot\left(\frac{n_{\epsilon}^{+}}{1+\epsilon e_{\epsilon}^{+}} \nabla c_{\epsilon}\right) \text { in } \Omega \times(0, T), \\
w_{\epsilon t}-\Delta w_{\epsilon}=-u_{\epsilon} \cdot \nabla w_{\epsilon}-\alpha \gamma w_{\epsilon}^{+} \frac{1}{\epsilon} \ln \left(1+\epsilon n_{\epsilon}^{+}\right)+\alpha g w_{\epsilon}^{+} \text {in } \Omega \times(0, T), \\
u_{\epsilon t}-\Delta u_{\epsilon}+\frac{1}{\alpha}\left(Y_{\epsilon} u_{\epsilon} \cdot \nabla\right) u_{\epsilon}+\nabla \pi_{\epsilon}=\alpha n_{\epsilon} \nabla \Phi+\alpha f \text { in } \Omega \times(0, T), \\
\nabla \cdot u_{\epsilon}=0 \text { in } \Omega \times(0, T) .
\end{array}\right.
$$

We first prove that $n_{\epsilon} \geq 0$. Let $\left[n_{\epsilon}, w_{\epsilon}, u_{\epsilon}, \pi_{\epsilon}\right]$ be a solution of (3.8). As we did in Step 1, the right hand side of $(3.8)_{1}$ is in $L^{5 / 3}\left(L^{5 / 3}\right)$. Moreover $n_{\epsilon} \in X \hookrightarrow L^{10 / 3}\left(L^{10 / 3}\right) \hookrightarrow L^{5 / 2}\left(L^{5 / 2}\right)$. Then, we can test (3.8) ${ }_{1}$ by $n_{\epsilon}^{-}=\min \left\{n_{\epsilon}, 0\right\} \leq 0$, and having into account that the condition $\nabla \cdot u_{\epsilon}=0$ implies $\int_{\Omega}\left(u_{\epsilon} \cdot \nabla n_{\epsilon}^{-}\right) n_{\epsilon}^{-}=0$, we get

$$
\frac{1}{2} \frac{\mathrm{d}}{\mathrm{d} t}\left\|n_{\epsilon}^{-}\right\|_{L^{2}}^{2}+\left\|\nabla n_{\epsilon}^{-}\right\|_{L^{2}}^{2}=\alpha \chi\left(\frac{n_{\epsilon}^{+}}{1+\epsilon n_{\epsilon}^{+}} \nabla c_{\epsilon}, \nabla n_{\epsilon}^{-}\right)=0
$$

in the scalar distribution sense on $(0, T)$ ( $c f$. [46], Chap. III, Lem. 1.1). This implies $n_{\epsilon}^{-}=0$ and then $n_{\epsilon} \geq 0$ a.e. in $\Omega \times(0, T)$. In a similar way, testing $(3.8)_{2}$ by $w_{\epsilon}^{-}$we get

$$
\frac{1}{2} \frac{\mathrm{d}}{\mathrm{d} t}\left\|w_{\epsilon}^{-}\right\|_{L^{2}}^{2}+\left\|\nabla w_{\epsilon}^{-}\right\|_{L^{2}}^{2}=-\alpha \gamma\left(w_{\epsilon}^{+} \frac{1}{\epsilon} \ln \left(1+\epsilon n_{\epsilon}^{+}\right), w_{\epsilon}^{-}\right)+\alpha\left(g w_{\epsilon}^{+}, w_{\epsilon}^{-}\right)=0
$$

in the scalar distribution sense on $(0, T)$, which gives that $w_{\epsilon} \geq 0$ a.e. in $\Omega \times(0, T)$. 
Now we prove that $w_{\epsilon}$ is bounded in $X$. For that, testing $(3.8)_{2}$ by $w_{\epsilon}$, integrating in $\Omega$, taking into account that $\nabla \cdot u_{\epsilon}=0$, and by the Gagliardo-Niremberg inequality [37], we get

$$
\begin{aligned}
\frac{1}{2} \frac{\mathrm{d}}{\mathrm{d} t}\left\|w_{\epsilon}\right\|_{L^{2}}^{2}+\left\|\nabla w_{\epsilon}\right\|_{L^{2}}^{2} & =-\alpha \gamma\left(w_{\epsilon} \frac{1}{\epsilon} \ln \left(1+\epsilon n_{\epsilon}\right), w_{\epsilon}\right)+\alpha\left(g w_{\epsilon}, w_{\epsilon}\right) \\
& \leq\left(g w_{\epsilon}, w_{\epsilon}\right) \\
& \leq\|g\|_{L^{5 / 2}}\left\|w_{\epsilon}\right\|_{L^{10 / 3}}^{2} \\
& \leq C\|g\|_{L^{5 / 2}}\left(\left\|w_{\epsilon}\right\|_{L^{2}}^{4 / 5}\left\|\nabla w_{\epsilon}\right\|_{L^{2}}^{6 / 5}+\left\|w_{\epsilon}\right\|_{L^{2}}^{2}\right) \\
& \leq C\|g\|_{L^{5 / 2}}\left\|w_{\epsilon}\right\|_{L^{2}}^{2}+C_{\delta_{1}}\|g\|_{L^{5 / 2}}^{5 / 2}\left\|w_{\epsilon}\right\|_{L^{2}}^{2}+\delta_{1}\left\|\nabla w_{\epsilon}\right\|_{L^{2}}^{2} .
\end{aligned}
$$

Thus, applying the Gronwall inequality in (3.9) and the convergence $w_{0, \epsilon} \rightarrow c_{0}$ in $W^{1, q}(\Omega)$, taking $\delta_{1}>0$ small enough, we get

$$
\left\|w_{\epsilon}\right\|_{L^{2}}^{2} \leq C\left\|w_{0, \epsilon}\right\|_{L^{2}}^{2} \exp \left(\int_{0}^{t}\left(\|g\|_{L^{5 / 2}}+\|g\|_{L^{5 / 2}}^{5 / 2}\right) \mathrm{d} s\right) \leq C\left(\left\|c_{0}\right\|_{L^{2}},\|g\|_{L^{5 / 2}\left(L^{5 / 2}\right)}\right),
$$

and then, integrating in time (3.9) we get $\left\|w_{\epsilon}\right\|_{L^{2}\left(H^{1}\right)} \leq C$.

Using the last estimate we are going to bound $n_{\epsilon}$ in $X$. Testing $(3.8)_{1}$ by $n_{\epsilon}$ and integrating in $\Omega$ we get

$$
\begin{aligned}
\frac{1}{2} \frac{\mathrm{d}}{\mathrm{d} t}\left\|n_{\epsilon}\right\|_{L^{2}}^{2}+\left\|\nabla n_{\epsilon}\right\|_{L^{2}}^{2} & =\alpha \chi\left(\frac{n_{\epsilon}}{1+\epsilon n_{\epsilon}} \nabla c_{\epsilon}, \nabla n_{\epsilon}\right) \\
& \leq \alpha \chi\left\|n_{\epsilon}\right\|_{L^{4}}\left\|\nabla c_{\epsilon}\right\|_{L^{4}}\left\|\nabla n_{\epsilon}\right\|_{L^{2}} \\
& \leq C \alpha \chi\left(\left\|n_{\epsilon}\right\|_{L^{2}}^{1 / 4}\left\|\nabla n_{\epsilon}\right\|_{L^{2}}^{3 / 4}+\left\|n_{\epsilon}\right\|_{L^{2}}\right)\left\|\nabla c_{\epsilon}\right\|_{L^{4}}\left\|\nabla n_{\epsilon}\right\|_{L^{2}} \\
& =C \alpha \chi\left\|n_{\epsilon}\right\|_{L^{2}}^{1 / 4}\left\|\nabla n_{\epsilon}\right\|_{L^{2}}^{7 / 4}\left\|\nabla c_{\epsilon}\right\|_{L^{4}}+C \alpha \chi\left\|n_{\epsilon}\right\|_{L^{2}}\left\|\nabla n_{\epsilon}\right\|_{L^{2}}\left\|\nabla c_{\epsilon}\right\|_{L^{4}} \\
& \leq \frac{1}{2}\left\|\nabla n_{\epsilon}\right\|_{L^{2}}^{2}+C\left\|n_{\epsilon}\right\|_{L^{2}}^{2}\left\|\nabla c_{\epsilon}\right\|_{L^{4}}^{8}+C\left\|n_{\epsilon}\right\|_{L^{2}}^{2}\left\|\nabla c_{\epsilon}\right\|_{L^{4}}^{2} .
\end{aligned}
$$

Since $c_{\epsilon}$ is the solution of (3.3) and $w_{\epsilon} \in X$, we know that $c_{\epsilon} \in L^{\infty}\left(H^{2}\right) \cap L^{2}\left(H^{3}\right)$ (cf. [27], Thms. 2.4.2.7 and 2.5.1.1), and

$$
\left\|c_{\epsilon}\right\|_{L^{\infty}\left(H^{2}\right) \cap L^{2}\left(H^{3}\right)} \leq C(\epsilon)\left\|w_{\epsilon}\right\|_{X} .
$$

In particular

$$
\left\|\nabla c_{\epsilon}\right\|_{L^{\infty}\left(L^{4}\right)} \leq C(\epsilon)\left\|w_{\epsilon}\right\|_{X} \leq C(\epsilon)
$$

Then, by applying the Gronwall inequality in (3.10) we get

$$
\left\|n_{\epsilon}\right\|_{X} \leq C(\epsilon) .
$$

Step four. $\Gamma: X \times X \rightarrow X \times X$ is continuous.

Let $\left[\bar{n}_{\epsilon}^{m}, \bar{w}_{\epsilon}^{m}\right]_{m \in \mathbb{N}} \subset X \times X$ be a sequence such that

$$
\left[\bar{n}_{\epsilon}^{m}, \bar{w}_{\epsilon}^{m}\right]_{m} \rightarrow\left[\bar{n}_{\epsilon}, \bar{w}_{\epsilon}\right] \text { in } X \times X \text { as } m \rightarrow \infty .
$$

In particular $\left[\bar{n}_{\epsilon}^{m}, \bar{w}_{\epsilon}^{m}\right]_{m \in \mathbb{N}}$ is bounded in $X \times X$, and by (3.6), (3.7), $\left[n_{\epsilon}^{m}, w_{\epsilon}^{m}\right]=\Gamma\left[\bar{n}_{\epsilon}^{m}, \bar{w}_{\epsilon}^{m}\right]$ is bounded in $\mathcal{X}_{5 / 3} \times \mathcal{X}_{5 / 3}$. Then there exists a subsequence, still denoted by $\left[n_{\epsilon}^{m}, w_{\epsilon}^{m}\right]_{m \in \mathbb{N}}$, and a pair of functions $\left[\hat{n}_{\epsilon}, \hat{w}_{\epsilon}\right] \in$ 
$\mathcal{X}_{5 / 3} \times \mathcal{X}_{5 / 3}$ such that

$$
\left[n_{\epsilon}^{m}, w_{\epsilon}^{m}\right]=\Gamma\left[\bar{n}_{\epsilon}^{m}, \bar{w}_{\epsilon}^{m}\right] \rightarrow\left[\hat{n}_{\epsilon}, \hat{w}_{\epsilon}\right] \text {, weakly in } \mathcal{X}_{5 / 3} \times \mathcal{X}_{5 / 3} \text { and strongly in } X \times X \text {. }
$$

Notice that equality $\left[n_{\epsilon}^{m}, w_{\epsilon}^{m}\right]=\Gamma\left[\bar{n}_{\epsilon}^{m}, \bar{w}_{\epsilon}^{m}\right]$ means

$$
\left\{\begin{array}{l}
n_{\epsilon t}^{m}-\Delta n_{\epsilon}^{m}=-u_{\epsilon}^{m} \cdot \nabla \bar{n}_{\epsilon}^{m}-\chi \nabla \cdot\left(\frac{\bar{n}_{\epsilon}^{m}}{1+\bar{n} \bar{n}_{\epsilon}^{m}} \nabla \bar{c}_{\epsilon}^{m}\right) \text { in } \Omega \times(0, T), \\
w_{\epsilon t}^{m}-\Delta w_{\epsilon}^{m}=-u_{\epsilon}^{m} \cdot \nabla \bar{w}_{\epsilon}^{m}-\gamma \bar{w}_{\epsilon}^{m} \frac{1}{\epsilon} \ln \left(1+\epsilon \bar{n}_{\epsilon}^{m}\right)+g \bar{w}_{\epsilon}^{m} \text { in } \Omega \times(0, T), \\
u_{\epsilon t}^{m}-\Delta u_{\epsilon}^{m}+\left(Y_{\epsilon} u_{\epsilon}^{m} \cdot \nabla\right) u_{\epsilon}^{m}+\nabla \pi_{\epsilon}^{m}=\bar{n}_{\epsilon}^{m} \nabla \Phi+f \text { in } \Omega \times(0, T), \\
\nabla \cdot u_{\epsilon}^{m}=0 \text { in } \Omega \times(0, T),
\end{array}\right.
$$

and the corresponding elliptic problem is satisfied

$$
\left\{\begin{array}{l}
\bar{c}_{\epsilon}^{m}-\epsilon \Delta \bar{c}_{\epsilon}^{m}=\bar{w}_{\epsilon}^{m} \text { in } \Omega \\
\frac{\partial \bar{c}_{\epsilon}^{m}(x, t)}{\partial \nu}=0 \text { on } \partial \Omega
\end{array}\right.
$$

As we did in step $\mathbf{1}$, since $\bar{n}_{\epsilon}^{m} \in X$, from $(3.12)_{3}$ we have $u_{\epsilon}^{m} \in \mathcal{X}_{2}$ and

$$
\begin{aligned}
\frac{1}{2} \frac{\mathrm{d}}{\mathrm{d} t}\left\|u_{\epsilon}^{m}\right\|_{L^{2}}^{2}+\left\|\nabla u_{\epsilon}^{m}\right\|_{L^{2}}^{2} & =\left(\bar{n}_{\epsilon}^{m} \nabla \Phi, u_{\epsilon}^{m}\right)+\left(f, u_{\epsilon}^{m}\right) \\
& \leq\|\nabla \Phi\|_{L^{\infty}}\left\|\bar{n}_{\epsilon}^{m}\right\|_{L^{2}}\left\|u_{\epsilon}^{m}\right\|_{L^{2}}+\|f\|_{L^{2}}\left\|u_{\epsilon}^{m}\right\|_{L^{2}}
\end{aligned}
$$

Since $\left\|\bar{n}_{\epsilon}^{m}\right\|_{X} \leq C(\epsilon)$, by the Gronwall inequality

$$
\left\|u_{\epsilon}^{m}\right\|_{X} \leq C(\epsilon)
$$

Also, testing $(3.12)_{3}$ by $A u_{\epsilon}^{m}$, we get

$$
\begin{aligned}
\frac{1}{2} \frac{\mathrm{d}}{\mathrm{d} t}\left\|\nabla u_{\epsilon}^{m}\right\|_{L^{2}}^{2}+\left\|A u_{\epsilon}^{m}\right\|_{L^{2}}^{2}= & -\left(\left(Y_{\epsilon} u_{\epsilon}^{m} \cdot \nabla\right) u_{\epsilon}^{m}, A u_{\epsilon}^{m}\right)+\left(\bar{n}_{\epsilon}^{m} \nabla \Phi, A u_{\epsilon}^{m}\right)+\left(f, A u_{\epsilon}^{m}\right) \\
\leq & \left\|Y_{\epsilon} u_{\epsilon}^{m}\right\|_{L^{4}}\left\|\nabla u_{\epsilon}^{m}\right\|_{L^{4}}\left\|A u_{\epsilon}^{m}\right\|_{L^{2}}+\|\nabla \Phi\|_{L^{\infty}}\left\|\bar{n}_{\epsilon}^{m}\right\|_{L^{2}}\left\|A u_{\epsilon}^{m}\right\|_{L^{2}} \\
& +\|f\|_{L^{2}}\left\|A u_{\epsilon}^{m}\right\|_{L^{2}} \\
\leq & 3 \delta_{2}\left\|A u_{\epsilon}^{m}\right\|_{L^{2}}^{2}+C_{\delta_{2}}\left\|Y_{\epsilon} u_{\epsilon}^{m}\right\|_{L^{4}}^{2}\left\|\nabla u_{\epsilon}^{m}\right\|_{L^{4}}^{2}+C_{\delta_{2}}\|\nabla \Phi\|_{L^{\infty}}^{2}\left\|\bar{n}_{\epsilon}^{m}\right\|_{L^{2}}^{2} \\
& +C_{\delta_{2}}\|f\|_{L^{2}}^{2} .
\end{aligned}
$$

Notice that

$$
\begin{aligned}
\left\|Y_{\epsilon} u_{\epsilon}^{m}\right\|_{L^{4}}^{2}\left\|\nabla u_{\epsilon}^{m}\right\|_{L^{4}}^{2} & \leq C\left\|Y_{\epsilon} u_{\epsilon}^{m}\right\|_{L^{4}}^{2}\left\|\nabla u_{\epsilon}^{m}\right\|_{L^{2}}^{1 / 2}\left\|A u_{\epsilon}^{m}\right\|_{L^{2}}^{3 / 2} \\
& \leq \delta_{3}\left\|A u_{\epsilon}^{m}\right\|_{L^{2}}^{2}+C_{\delta_{3}}\left\|Y_{\epsilon} u_{\epsilon}^{m}\right\|_{L^{4}}^{8}\left\|\nabla u_{\epsilon}^{m}\right\|_{L^{2}}^{2}
\end{aligned}
$$

Then, from last two inequalities we get

$$
\frac{1}{2} \frac{\mathrm{d}}{\mathrm{d} t}\left\|\nabla u_{\epsilon}^{m}\right\|_{L^{2}}^{2}+\left(1-3 \delta_{2}-\delta_{3}\right)\left\|A u_{\epsilon}^{m}\right\|_{L^{2}}^{2} \leq C_{\delta_{2}, \delta_{3}}\left\|Y_{\epsilon} u_{\epsilon}^{m}\right\|_{L^{4}}^{8}\left\|\nabla u_{\epsilon}^{m}\right\|_{L^{2}}^{2}+C_{\delta_{2}}\|\nabla \Phi\|_{L^{\infty}}^{2}\left\|\bar{n}_{\epsilon}^{m}\right\|_{L^{2}}^{2}+C_{\delta_{2}}\|f\|_{L^{2}}^{2} .
$$

Since the Yosida approximation $Y_{\epsilon} u_{\epsilon}^{m}$ is in $L^{8}\left(L^{4}\right)$ (cf. [43], Chap. 5), by the Gronwall inequality

$$
\left\|u_{\epsilon}^{m}\right\|_{\mathcal{X}_{2}} \leq C(\epsilon)
$$


From (3.14), (3.15), there exists $u_{\epsilon} \in \mathcal{X}_{2}$ such that

$$
u_{\epsilon}^{m} \rightarrow u_{\epsilon} \text {, weakly in } \mathcal{X}_{2} \text { and strongly in } X .
$$

Therefore, from (3.11) and (3.16) we can pass to the limit, as $m \rightarrow \infty$, in system (3.12). Strong estimates for $n_{\epsilon}^{m}$ and $w_{\epsilon}^{m}$ in $\mathcal{X}_{5 / 3}$ allow us to pass to the limit in $(3.12)_{1,2}$. In order to pass to the limit in the velocity equation it is enough to see that

$$
\left(Y_{\epsilon} u_{\epsilon}^{m} \cdot \nabla\right) u_{\epsilon}^{m}-\left(Y_{\epsilon} u_{\epsilon} \cdot \nabla\right) u_{\epsilon}=Y_{\epsilon}\left(u_{\epsilon}^{m}-u\right) \cdot \nabla u_{\epsilon}^{m}+Y_{\epsilon} u \cdot \nabla\left(u_{\epsilon}^{m}-u\right)
$$

and that $\left\|Y_{\epsilon}\left(u_{\epsilon}^{m}-u\right)\right\|_{L^{2}} \leq\left\|u_{\epsilon}^{m}-u\right\|_{L^{2}},\left\|Y_{\epsilon} u\right\|_{L^{2}} \leq\|u\|_{L^{2}}$ (cf. [43], Chap. V). At the limit we get

$$
\left\{\begin{array}{l}
\hat{n}_{\epsilon t}-\Delta \hat{n}_{\epsilon}=-u_{\epsilon} \cdot \nabla \bar{n}_{\epsilon}-\chi \nabla \cdot\left(\frac{\bar{n}_{\epsilon}}{1+\epsilon \bar{n}_{\epsilon}} \nabla \bar{c}_{\epsilon}\right) \text { in } \Omega \times(0, T), \\
\hat{w}_{\epsilon t}-\Delta \hat{w}_{\epsilon}=-u_{\epsilon} \cdot \nabla \bar{w}_{\epsilon}-\gamma \bar{w}_{\epsilon} \frac{1}{\epsilon} \ln \left(1+\epsilon \bar{n}_{\epsilon}\right)+g \bar{w}_{\epsilon} \text { in } \Omega \times(0, T), \\
u_{\epsilon t}-\Delta u_{\epsilon}+\left(Y_{\epsilon} u_{\epsilon} \cdot \nabla\right) u_{\epsilon}+\nabla \pi_{\epsilon}=\bar{n}_{\epsilon} \nabla \Phi+f \text { in } \Omega \times(0, T), \\
\nabla \cdot u_{\epsilon}=0 \text { in } \Omega \times(0, T),
\end{array}\right.
$$

where $\bar{c}_{\epsilon}$ is the unique solution of the elliptic problem (3.3) with right hand side $\bar{w}_{\epsilon}$. Namely, $\left[\hat{n}_{\epsilon}, \hat{w}_{\epsilon}\right]=\Gamma\left[\bar{n}_{\epsilon}, \bar{w}_{\epsilon}\right]$. We have proved that, for an arbitrary sequence $\left[\bar{n}_{\epsilon}^{m}, \bar{w}_{\epsilon}^{m}\right]_{m \in \mathbb{N}} \subset X \times X$, such that $\left[\bar{n}_{\epsilon}^{m}, \bar{w}_{\epsilon}^{m}\right]_{m} \rightarrow\left[\bar{n}_{\epsilon}, \bar{w}_{\epsilon}\right]$, there exists a subsequence of $\left[\bar{n}_{\epsilon}^{m}, \bar{w}_{\epsilon}^{m}\right]_{m \in \mathbb{N}}$, still denoted by $\left[\bar{n}_{\epsilon}^{m}, \bar{w}_{\epsilon}^{m}\right]_{m \in \mathbb{N}}$, such that $\Gamma\left[\bar{n}_{\epsilon}^{m}, \bar{w}_{\epsilon}^{m}\right]_{m \in \mathbb{N}} \rightarrow \Gamma\left[\bar{n}_{\epsilon}, \bar{w}_{\epsilon}\right]$. This implies the continuity of $\Gamma$.

From the previous steps the operator $\Gamma$ satisfies the hypothesis of the Leray-Schauder fixed point theorem. Thus, we conclude that the map $\Gamma$ has a fixed point $\left[n_{\epsilon}, w_{\epsilon}\right]$, that is, $\Gamma\left[n_{\epsilon}, w_{\epsilon}\right]=\left[n_{\epsilon}, w_{\epsilon}\right]$, which provides a solution of system (3.2).

Step five. Uniqueness of the solution.

We proceed as follows. First, observe that

$$
n_{\epsilon} \in \mathcal{X}_{5 / 3} \subset L^{\infty}\left(W^{4 / 5,5 / 3}\right) \cap L^{5 / 3}\left(W^{2,5 / 3}\right) \hookrightarrow L^{\infty}\left(L^{3}\right) \cap L^{2}\left(L^{\infty}\right),
$$

where we have used the classical Sobolev embedding and Lemma A.4 with $q_{1}=3, p_{1}=p_{2}=5 / 3, r=2, p=2$. Second, since $n_{\epsilon} \in L^{\infty}\left(W^{4 / 5,5 / 3}\right) \cap L^{5 / 3}\left(W^{2,5 / 3}\right)$, from (A.1) in the appendix we have

$$
n_{\epsilon} \in L^{\infty}\left(H^{1 / 2}\right) \cap L^{5 / 3}\left(H^{17 / 10}\right) .
$$

Then, from Lemma A.3 with $\left(p_{1}, s_{1}\right)=(\infty, 1 / 2),\left(p_{2}, s_{2}\right)=(5 / 3,17 / 10)$ we get $n_{\epsilon} \in L^{20 / 9}\left(H^{7 / 5}\right)$. In particular $\nabla n_{\epsilon} \in L^{20 / 9}\left(H^{2 / 5}\right) \hookrightarrow L^{20 / 9}\left(L^{20 / 9}\right)$.

Let us assume that $\left[n_{\epsilon, 1}, w_{\epsilon, 1}, u_{\epsilon, 1}, \pi_{\epsilon, 1}\right],\left[n_{\epsilon, 2}, w_{\epsilon, 2}, u_{\epsilon, 2}, \pi_{\epsilon, 2}\right]$ in $\mathcal{X}_{5 / 3} \times \mathcal{X}_{5 / 3} \times \mathcal{X}_{2} \times L^{2}\left(H^{1}\right)$, with $n_{\epsilon, 1}, n_{\epsilon, 2} \geq 0$, $w_{\epsilon, 1}, w_{\epsilon, 2} \geq 0$, in accordance with $c_{\epsilon, 1}, c_{\epsilon, 2} \in L^{\infty}\left(H^{2}\right) \cap L^{2}\left(H^{3}\right)$, are two solutions of (3.2). Then, if we define $u=u_{\epsilon, 1}-u_{\epsilon, 2}, n=n_{\epsilon, 1}-n_{\epsilon, 2}, w=w_{\epsilon, 1}-w_{\epsilon, 2}, c=c_{\epsilon, 1}-c_{\epsilon, 2}$ and $\pi=\pi_{\epsilon, 1}-\pi_{\epsilon, 2}$, we have

$$
\left\{\begin{array}{l}
u_{t}-\Delta u+\left(Y_{\epsilon} u \cdot \nabla\right) u_{\epsilon, 1}+\left(Y_{\epsilon} u_{\epsilon, 2} \cdot \nabla\right) u+\nabla \pi=n \nabla \Phi, \\
\nabla \cdot u=0,
\end{array}\right.
$$

with $u(0)=0$, and $u=0$ on $\partial \Omega \times(0, T)$.

Notice that, since $W^{1,2}(\Omega) \subset L^{6}(\Omega)$ and taking into account the continuity of $Y_{\epsilon}$ on $L_{\sigma}^{2}(\Omega)$ (cf. [43], II.2.3.6), there exists $C>0$ such that for any $\epsilon>0$,

$$
\left\|Y_{\epsilon} u_{\epsilon}\right\|_{L^{6}} \leq C\left\|\nabla Y_{\epsilon} u_{\epsilon}\right\|_{L^{2}}=C\left\|A^{1 / 2} Y_{\epsilon} u_{\epsilon}\right\|_{L^{2}}=C\left\|Y_{\epsilon} A^{1 / 2} u_{\epsilon}\right\|_{L^{2}} \leq C\left\|A^{1 / 2} u_{\epsilon}\right\|_{L^{2}}=C\left\|\nabla u_{\epsilon}\right\|_{L^{2}} .
$$


Thus, $\left\{Y_{\epsilon} u_{\epsilon}\right\}$ is uniformly bounded in $L^{2}\left(L^{6}\right)$. Hence, if we multiply (3.17) by $u$, and integrate in $\Omega$, we get

$$
\begin{aligned}
\frac{1}{2} \frac{\mathrm{d}}{\mathrm{d} t}\|u\|_{L^{2}}^{2}+\|\nabla u\|_{L^{2}}^{2} & \leq\left\|Y_{\epsilon} u\right\|_{L^{6}}\left\|\nabla u_{\epsilon, 1}\right\|_{L^{2}}\|u\|_{L^{3}}+\|\nabla \Phi\|_{L^{\infty}}\|n\|_{L^{2}}\|u\|_{L^{2}} \\
& \leq C\|\nabla u\|_{L^{2}}\left\|\nabla u_{\epsilon, 1}\right\|_{L^{2}}\|u\|_{L^{2}}^{1 / 2}\|\nabla u\|_{L^{2}}^{1 / 2}+\|\nabla \Phi\|_{L^{\infty}}\|n\|_{L^{2}}\|u\|_{L^{2}} \\
& \leq C\left\|\nabla u_{\epsilon, 1}\right\|_{L^{2}}\|u\|_{L^{2}}^{1 / 2}\|\nabla u\|_{L^{2}}^{3 / 2}+\|\nabla \Phi\|_{L^{\infty}}\|n\|_{L^{2}}\|u\|_{L^{2}} \\
& \leq \delta_{4}\|\nabla u\|_{L^{2}}^{2}+C_{\delta_{4}}\left\|\nabla u_{\epsilon, 1}\right\|_{L^{2}}^{4}\|u\|_{L^{2}}^{2}+\frac{1}{2}\|\nabla \Phi\|_{L^{\infty}}^{2}\|n\|_{L^{2}}^{2}+\frac{1}{2}\|u\|_{L^{2}}^{2} .
\end{aligned}
$$

On the other hand,

$$
n_{t}-\Delta n+u_{\epsilon, 1} \cdot \nabla n=-u \cdot \nabla n_{\epsilon, 2}-\chi \nabla \cdot\left[\frac{n_{\epsilon, 1}}{1+\epsilon n_{\epsilon, 1}} \nabla c+\left(\frac{n_{\epsilon, 1}}{1+\epsilon n_{\epsilon, 1}}-\frac{n_{\epsilon, 2}}{1+\epsilon n_{\epsilon, 2}}\right) \nabla c_{\epsilon, 2}\right],
$$

with $n(0)=0$, and $\frac{\partial n}{\partial \nu}=0$ on $\partial \Omega \times(0, T)$. Thus, multiplying by $n$, integrating in $\Omega$, and using the GagliardoNiremberg inequality (as in (3.10)) we obtain

$$
\begin{aligned}
\frac{1}{2} \frac{\mathrm{d}}{\mathrm{d} t} \| & n\left\|_{L^{2}}^{2}+\right\| \nabla n \|_{L^{2}}^{2} \\
\leq & -\int_{\Omega}\left(u \cdot \nabla n_{\epsilon, 2}\right) n+\chi \int_{\Omega} \frac{n_{\epsilon, 1}}{1+\epsilon n_{\epsilon, 1}} \nabla c \cdot \nabla n+\chi \int_{\Omega} \frac{n_{\epsilon, 1}-n_{\epsilon, 2}}{\left(1+\epsilon n_{\epsilon, 1}\right)\left(1+\epsilon n_{\epsilon, 2}\right)} \nabla c_{\epsilon, 2} \cdot \nabla n \\
\leq & \int_{\Omega} n_{\epsilon, 2} u \cdot \nabla n+\chi\|\nabla c\|_{L^{2}}\|\nabla n\|_{L^{2}}+\chi\left\|\nabla c_{\epsilon, 2}\right\|_{L^{4}}\|\nabla n\|_{L^{2}}\|n\|_{L^{4}} \\
\leq & \left\|n_{\epsilon, 2}\right\|\left\|_{L^{\infty}}\right\| u\left\|_{L^{2}}\right\| \nabla n\left\|_{L^{2}}+\chi\right\| \nabla c\left\|_{L^{2}}\right\| \nabla n \|_{L^{2}} \\
& +\chi\left\|\nabla c_{\epsilon, 2}\right\|_{L^{4}}\|\nabla n\|_{L^{2}}^{7 / 4}\|n\|_{L^{2}}^{1 / 4}+\chi\left\|\nabla c_{\epsilon, 2}\right\|_{L^{4}}\|\nabla n\|_{L^{2}}\|n\|_{L^{2}} \\
\leq & \delta_{5}\|\nabla n\|_{L^{2}}^{2}+C_{\delta_{5}}\left\|n_{\epsilon, 2}\right\|_{L^{\infty}}^{2}\|u\|_{L^{2}}^{2}+C_{\delta_{5}}\|\nabla c\|_{L^{2}}^{2}+C_{\delta_{5}}\left\|\nabla c_{\epsilon, 2}\right\|_{L^{4}}^{8}\|n\|_{L^{2}}^{2} \\
\leq & \delta_{5}\|\nabla n\|_{L^{2}}^{2}+C_{\delta_{5}}\left\|n_{\epsilon, 2}\right\|_{L^{\infty}}^{2}\|u\|_{L^{2}}^{2}+C_{\delta_{5}}\|\nabla w\|_{L^{2}}^{2}+C_{\delta_{5}}\left\|\nabla c_{\epsilon, 2}\right\|_{L^{4}}^{8}\|n\|_{L^{2}}^{2},
\end{aligned}
$$

where in the last inequality we have used (3.3). On the other hand,

$$
w_{t}-\Delta w+u_{\epsilon, 1} \cdot \nabla w=-u \cdot \nabla w_{\epsilon, 2}-\frac{\gamma}{\epsilon} w_{\epsilon, 1} \ln \left(1+\epsilon n_{\epsilon, 1}\right)+\frac{\gamma}{\epsilon} w_{\epsilon, 2} \ln \left(1+\epsilon n_{\epsilon, 2}\right)+g w,
$$

with $w(0)=0$, and $\frac{\partial w}{\partial \nu}=0$ on $\partial \Omega \times(0, T)$. Observe that

$$
\begin{aligned}
-\frac{\gamma}{\epsilon} w_{\epsilon, 1} \ln \left(1+\epsilon n_{\epsilon, 1}\right) & +\frac{\gamma}{\epsilon} w_{\epsilon, 2} \ln \left(1+\epsilon n_{\epsilon, 2}\right) \\
& =-\frac{\gamma}{\epsilon} w \ln \left(1+\epsilon n_{\epsilon, 1}\right)-w_{\epsilon, 2}\left(\ln \left(1+\epsilon n_{\epsilon, 1}\right)-\ln \left(1+\epsilon n_{\epsilon, 2}\right)\right) \\
& =-\frac{\gamma}{\epsilon} w \ln \left(1+\epsilon n_{\epsilon, 1}\right)-w_{\epsilon, 2} \ln \left(\frac{\epsilon n}{1+\epsilon n_{\epsilon, 2}}+1\right) .
\end{aligned}
$$

Therefore, testing (3.21) by $w$, and working as in (3.9) we get

$$
\begin{aligned}
& \frac{1}{2} \frac{\mathrm{d}}{\mathrm{d} t}\|w\|_{L^{2}}^{2}+\|\nabla w\|_{L^{2}}^{2} \\
& \quad \leq \int_{\Omega} w_{\epsilon, 2} u \cdot \nabla w+\gamma \int_{\Omega} w^{2} n_{\epsilon, 1}+\gamma \int_{\Omega} w_{\epsilon, 2} n w+\int_{\Omega} g w^{2}
\end{aligned}
$$




$$
\begin{aligned}
\leq & \delta_{6}\|\nabla w\|_{L^{2}}^{2}+C_{\delta_{6}}\left\|w_{\epsilon, 2}\right\|_{L^{\infty}}^{2}\|u\|_{L^{2}}^{2}+\gamma\left\|n_{\epsilon, 1}\right\|_{L^{\infty}}\|w\|_{L^{2}}^{2}+\frac{\gamma}{2}\left\|w_{\epsilon, 2}\right\|_{L^{\infty}}\|n\|_{L^{2}}^{2}+\frac{\gamma}{2}\|w\|_{L^{2}}^{2} \\
& +\delta_{7}\|\nabla w\|_{L^{2}}^{2}+C\|g\|_{L^{5 / 2}}\|w\|_{L^{2}}^{2}+C_{\delta_{7}}\|g\|_{L^{5 / 2}}^{5 / 2}\|w\|_{L^{2}}^{2} .
\end{aligned}
$$

Finally, multiplying (3.22) by $1+C_{\delta_{5}}$, and adding to (3.19), (3.20), gives

$$
\begin{aligned}
\frac{1}{2} \frac{\mathrm{d}}{\mathrm{d} t}( & \left.\|n\|_{L^{2}}^{2}+\left(C_{\delta_{5}}+1\right)\|w\|_{L^{2}}^{2}+\|u\|_{L^{2}}^{2}\right)+\left(1-\delta_{5}\right)\|\nabla n\|_{L^{2}}^{2}+\left(1-\left(\delta_{6}+\delta_{7}\right)\left(1+C_{\delta_{5}}\right)\right)\|\nabla w\|_{L^{2}}^{2} \\
& +\left(1-\delta_{4}\right)\|\nabla u\|_{L^{2}}^{2} \leq C\left(C_{\delta_{5}}\left\|\nabla c_{\epsilon, 2}\right\|_{L^{4}}^{8}+\frac{\gamma}{2}\left\|w_{\epsilon, 2}\right\|_{L^{\infty}}+\gamma\left\|n_{\epsilon, 1}\right\|_{L^{\infty}}+C\|g\|_{L^{5 / 2}}+C_{\delta_{7}}\|g\|_{L^{5 / 2}}^{5 / 2}\right. \\
& \left.+C_{\delta_{4}}\left\|\nabla u_{\epsilon, 1}\right\|_{L^{2}}^{4}+C_{\delta_{5}}\left\|n_{\epsilon, 2}\right\|_{L^{\infty}}^{2}+C_{\delta_{6}}\left\|w_{\epsilon, 2}\right\|_{L^{\infty}}^{2}+\frac{\gamma+1}{2}\right)\left(\|n\|_{L^{2}}^{2}+\left(C_{\delta_{5}}+1\right)\|w\|_{L^{2}}^{2}+\|u\|_{L^{2}}^{2}\right),
\end{aligned}
$$

and then, since $n(0)=w(0)=u(0)$, by the Gronwall inequality we get $n=w=u=0$, which proves the uniqueness.

\subsection{Uniform estimates}

The aim of this section is to find proper estimates, independent of $\epsilon$, allowing the pass to the limit in $(3.2)-(3.3)$.

Lemma 3.6. Let $\left[n_{\epsilon}, w_{\epsilon}, u_{\epsilon}, \pi_{\epsilon}\right], n_{\epsilon} \geq 0, w_{\epsilon} \geq 0$, be a strong solution of (3.2) and $g \in L^{5 / 2}\left(L^{5 / 2}\right)$. Then, $w_{\epsilon} \in$ $\mathcal{X}_{5 / 3}$ satisfying

$$
\left\{\begin{array}{l}
w_{\epsilon t}-\Delta w_{\epsilon}=-u_{\epsilon} \cdot \nabla w_{\epsilon}-\gamma w_{\epsilon} \frac{1}{\epsilon} \ln \left(1+\epsilon n_{\epsilon}\right)+g w_{\epsilon}, \text { in } \Omega \times(0, T) \\
\frac{\partial w_{\epsilon}}{\partial \nu}=0, \text { on } \partial \Omega \times(0, T), \\
w_{\epsilon}(0)=w_{0, \epsilon} \in W^{1, q}(\Omega), q>3
\end{array}\right.
$$

verifies that

$$
0 \leq w_{\epsilon}(x, t) \leq C\left(\left\|c_{0}\right\|_{L^{p}},\|g\|_{L^{5 / 2}\left(L^{5 / 2}\right)}\right)
$$

Proof. From Step three in the proof of Proposition 3.5 we known that $w_{\epsilon} \geq 0$. Now, by multiplying (3.23) ${ }_{1}$ by $\frac{1}{p} w_{\epsilon}^{p-1}$ and integrating in $\Omega$, we have

$$
\frac{1}{p^{2}} \frac{\mathrm{d}}{\mathrm{d} t} \int_{\Omega}\left(w_{\epsilon}^{p / 2}\right)^{2}-\frac{1}{p} \int_{\Omega} \Delta w_{\epsilon} w_{\epsilon}^{p-1}+\frac{1}{p} \int_{\Omega} u_{\epsilon} \cdot \nabla w_{\epsilon} w_{\epsilon}^{p-1}=-\frac{\gamma}{p \epsilon} \int_{\Omega} \ln \left(1+\epsilon n_{\epsilon}\right) w_{\epsilon}^{p}+\frac{1}{p} \int_{\Omega} g w_{\epsilon}^{p} .
$$

Then, since $\nabla \cdot u_{\epsilon}=0$, and by the boundary condition $\left.u\right|_{\partial \Omega}=0$, the third term in the left hand side of last equality is zero. Thus, after integration by parts, we obtain

$$
\frac{1}{p^{2}} \frac{\mathrm{d}}{\mathrm{d} t} \int_{\Omega}\left(w_{\epsilon}^{p / 2}\right)^{2}+\frac{p-1}{p} \frac{4}{p^{2}} \int_{\Omega}\left|\nabla\left(w_{\epsilon}^{p / 2}\right)\right|^{2}=-\frac{\gamma}{p \epsilon} \int_{\Omega} \ln \left(1+\epsilon n_{\epsilon}\right) w_{\epsilon}^{p}+\frac{1}{p} \int_{\Omega} g w_{\epsilon}^{p} .
$$

Therefore, by making the substitution $\omega=w_{\epsilon}^{p / 2}$ and since $w_{\epsilon} \geq 0$, by the Gagliardo-Niremberg inequality, as in (3.9), we get

$$
\frac{\mathrm{d}}{\mathrm{d} t}\|\omega\|_{L^{2}}^{2}+\frac{4(p-1)}{p}\|\nabla \omega\|_{L^{2}}^{2} \leq p \int_{\Omega} g \omega^{2} \leq p\|g\|_{L^{5 / 2}}\|\omega\|_{L^{10 / 3}}^{2}
$$




$$
\leq p \delta\|\nabla \omega\|_{L^{2}}^{2}+C_{\delta}\|g\|_{L^{5 / 2}}^{5 / 2}\|\omega\|_{L^{2}}^{2}+C\|g\|_{L^{5 / 2}}\|\omega\|_{L^{2}}^{2} .
$$

Thus, taking $\delta$ small enough and then applying the Gronwall inequality, we have

$$
\left\|w_{\epsilon}\right\|_{L^{\infty}\left(L^{p}\right)} \leq C\left(\left\|w_{0, \epsilon}\right\|_{L^{p}},\|g\|_{L^{5 / 2}\left(L^{5 / 2}\right)}\right) \leq C\left(\left\|c_{0}\right\|_{L^{p}},\|g\|_{L^{5 / 2}\left(L^{5 / 2}\right)}\right) .
$$

Using that

$$
\left\|w_{0, \epsilon}\right\|_{L^{p}} \leq|\Omega|^{1 / p}\left\|w_{0, \epsilon}\right\|_{L^{\infty}} \leq C\left\|w_{0, \epsilon}\right\|_{W^{1, q}}
$$

from (3.26), we conclude that

$$
\left\|w_{\epsilon}\right\|_{L^{\infty}\left(L^{p}\right)} \leq C\left(\left\|w_{0, \epsilon}\right\|_{W^{1, q}},\|g\|_{L^{5 / 2}\left(L^{5 / 2}\right)}\right) .
$$

Moreover, going back to (3.25), we obtain $\|\nabla \omega\|_{L^{2}\left(L^{2}\right)} \leq C\left(p,\|g\|_{L^{5 / 2}\left(L^{5 / 2}\right)},\left\|\omega_{0}\right\|_{L^{2}}\right)$, that is,

$$
w_{\epsilon} \in L^{\infty}\left(L^{p}\right) \cap L^{p}\left(W^{1, p}\right), \quad \text { for any } p>1 .
$$

From (3.27), and Theorem 2.8 of [1] we obtain that $w_{\epsilon}$ is uniformly bounded in $L^{\infty}\left(L^{\infty}\right)$ and thus, we conclude the proof of (3.24).

Next, we are going to derive a quasi-energy estimate for system (3.2), which is the key for deriving strong convergences allowing the pass to the limit as $\epsilon \rightarrow 0$. For that we test system (3.2) with suitable functions in such a way that the regularized chemotaxis term is absorbed by the regularized consumption signal. First, multiplying $(3.2)_{1}$ by $\ln n_{\epsilon}$, integrating in $\Omega$, and using the mass conservation property for $n_{\epsilon}$ (we already proved $\left.n_{\epsilon}^{+}=n_{\epsilon}\right)$ gives

$$
\frac{\mathrm{d}}{\mathrm{d} t} \int_{\Omega} n_{\epsilon} \ln n_{\epsilon}=-\int_{\Omega} \frac{\left|\nabla n_{\epsilon}\right|^{2}}{n_{\epsilon}}+\chi \int_{\Omega} \frac{\nabla n_{\epsilon} \cdot \nabla c_{\epsilon}}{1+\epsilon n_{\epsilon}}, \quad \text { on }(0, T) .
$$

Let us compute now $\frac{\mathrm{d}}{\mathrm{d} t} \int_{\Omega} \frac{\left|\nabla w_{\epsilon}\right|^{2}}{w_{\epsilon}}$, for any $\epsilon>0$ on $(0, T)$. By using $(3.2)_{2}$, and following Lemma 2.8 in [34] one has

$$
\begin{aligned}
\frac{\mathrm{d}}{\mathrm{d} t} \int_{\Omega} \frac{\left|\nabla w_{\epsilon}\right|^{2}}{w_{\epsilon}}= & -2 \int_{\Omega} \frac{\left|\Delta w_{\epsilon}\right|^{2}}{w_{\epsilon}}+2 \int_{\Omega} \frac{\Delta w_{\epsilon}}{w_{\epsilon}} u_{\epsilon} \cdot \nabla w_{\epsilon}+2 \gamma \int_{\Omega} \Delta w_{\epsilon} \frac{1}{\epsilon} \ln \left(1+\epsilon n_{\epsilon}\right) \\
& -2 \int_{\Omega} \Delta w_{\epsilon} g+\int_{\Omega} \frac{\left|\nabla w_{\epsilon}\right|^{2}}{w_{\epsilon}^{2}} \Delta w_{\epsilon}-\int_{\Omega} \frac{\left|\nabla w_{\epsilon}\right|^{2}}{w_{\epsilon}^{2}} u_{\epsilon} \cdot \nabla w_{\epsilon} \\
& -\gamma \int_{\Omega} \frac{\left|\nabla w_{\epsilon}\right|^{2}}{w_{\epsilon}} \frac{1}{\epsilon} \ln \left(1+\epsilon n_{\epsilon}\right)+\int_{\Omega} \frac{\left|\nabla w_{\epsilon}\right|^{2}}{w_{\epsilon}} g \\
\leq & -2 \int_{\Omega} \frac{\left|\Delta w_{\epsilon}\right|^{2}}{w_{\epsilon}}+2 \int_{\Omega} \frac{\Delta w_{\epsilon}}{w_{\epsilon}} u_{\epsilon} \cdot \nabla w_{\epsilon}-2 \gamma \int_{\Omega} \frac{\nabla w_{\epsilon} \cdot \nabla n_{\epsilon}}{1+\epsilon n_{\epsilon}}-2 \int_{\Omega} \Delta w_{\epsilon} g \\
& +\int_{\Omega} \frac{\left|\nabla w_{\epsilon}\right|^{2}}{w_{\epsilon}^{2}} \Delta w_{\epsilon}-\int_{\Omega} \frac{\left|\nabla w_{\epsilon}\right|^{2}}{w_{\epsilon}^{2}} u_{\epsilon} \cdot \nabla w_{\epsilon}+\int_{\Omega} \frac{\left|\nabla w_{\epsilon}\right|^{2}}{w_{\epsilon}} g .
\end{aligned}
$$

Now, by replacing $w_{\epsilon}$ from $(3.3)_{1}$, we get

$$
\frac{\mathrm{d}}{\mathrm{d} t} \int_{\Omega} \frac{\left|\nabla w_{\epsilon}\right|^{2}}{w_{\epsilon}} \leq-2 \int_{\Omega} \frac{\left|\Delta w_{\epsilon}\right|^{2}}{w_{\epsilon}}+2 \int_{\Omega} \frac{\Delta w_{\epsilon}}{w_{\epsilon}} u_{\epsilon} \cdot \nabla w_{\epsilon}-2 \gamma \int_{\Omega} \frac{\nabla c_{\epsilon} \cdot \nabla n_{\epsilon}}{1+\epsilon n_{\epsilon}}
$$




$$
\begin{aligned}
& +2 \gamma \epsilon \int_{\Omega} \frac{\nabla\left(\Delta c_{\epsilon}\right) \cdot \nabla n_{\epsilon}}{1+\epsilon n_{\epsilon}}-2 \int_{\Omega} \Delta w_{\epsilon} g+\int_{\Omega} \frac{\left|\nabla w_{\epsilon}\right|^{2}}{w_{\epsilon}^{2}} \Delta w_{\epsilon} \\
& -\int_{\Omega} \frac{\left|\nabla w_{\epsilon}\right|^{2}}{w_{\epsilon}^{2}} u_{\epsilon} \cdot \nabla w_{\epsilon}+\int_{\Omega} \frac{\left|\nabla w_{\epsilon}\right|^{2}}{w_{\epsilon}} g .
\end{aligned}
$$

By Lemma 2.7(vi) in [34], there exist $k_{1}>0, k_{2}>0$ such that for any $\epsilon>0$ :

$$
-2 \int_{\Omega} \frac{\left|\Delta w_{\epsilon}\right|^{2}}{w_{\epsilon}}+\int_{\Omega} \frac{\left|\nabla w_{\epsilon}\right|^{2}}{w_{\epsilon}^{2}} \Delta w_{\epsilon} \leq-k_{1} \int_{\Omega} w_{\epsilon}\left|D^{2} \ln w_{\epsilon}\right|^{2}-k_{1} \int_{\Omega} \frac{\left|\nabla w_{\epsilon}\right|^{4}}{w_{\epsilon}^{3}}+k_{2} \int_{\Omega} w_{\epsilon} .
$$

Having into account identity $\Delta w_{\epsilon}=w_{\epsilon} \Delta \ln w_{\epsilon}+\frac{\left|\nabla w_{\epsilon}\right|^{2}}{w_{\epsilon}}$, we have

$$
\begin{aligned}
-2 \int_{\Omega} \Delta w_{\epsilon} g+\int_{\Omega} \frac{\left|\nabla w_{\epsilon}\right|^{2}}{w_{\epsilon}} g & =-2 \int_{\Omega} w_{\epsilon} g \Delta \ln w_{\epsilon}-\int_{\Omega} \frac{\left|\nabla w_{\epsilon}\right|^{2}}{w_{\epsilon}} g \\
& \leq 2\left\|w_{\epsilon}^{1 / 2} D^{2} \ln w_{\epsilon}\right\|_{L^{2}}\left\|w_{\epsilon}^{1 / 2} g\right\|_{L^{2}}+\left\|\frac{\left|\nabla w_{\epsilon}\right|^{2}}{w_{\epsilon}^{3 / 2}}\right\|_{L^{2}}\left\|w_{\epsilon}^{1 / 2} g\right\|_{L^{2}} \\
& \leq \delta_{1} \int_{\Omega} w_{\epsilon}\left|D^{2} \ln w_{\epsilon}\right|^{2}+\delta_{2} \int_{\Omega} \frac{\left|\nabla w_{\epsilon}\right|^{4}}{w_{\epsilon}^{3}}+C_{\delta_{1}, \delta_{2}}\left\|w_{\epsilon}^{1 / 2} g\right\|_{L^{2}}^{2} \\
& \leq \delta_{1} \int_{\Omega} w_{\epsilon}\left|D^{2} \ln w_{\epsilon}\right|^{2}+\delta_{2} \int_{\Omega} \frac{\left|\nabla w_{\epsilon}\right|^{4}}{w_{\epsilon}^{3}}+C_{\delta_{1}, \delta_{2}} \int_{\Omega}\left|w_{\epsilon}\right|^{5}+C_{\delta_{1}, \delta_{2}} \int_{\Omega}|g|^{5 / 2} .
\end{aligned}
$$

Moreover, following the proof of Lemma 2.8 in [34], there exists $k_{3}>0$ such that for any $\epsilon>0$ :

$$
2 \int_{\Omega} \frac{\Delta w_{\epsilon}}{w_{\epsilon}} u_{\epsilon} \cdot \nabla w_{\epsilon}-\int_{\Omega} \frac{\left|\nabla w_{\epsilon}\right|^{2}}{w_{\epsilon}^{2}} u_{\epsilon} \cdot \nabla w_{\epsilon} \leq \frac{k_{1}}{2} \int_{\Omega} \frac{\left|\nabla w_{\epsilon}\right|^{4}}{w_{\epsilon}^{3}}+k_{3} \int_{\Omega} w_{\epsilon}\left|\nabla u_{\epsilon}\right|^{2}
$$

Then, putting together (3.30)-(3.33), we obtain

$$
\begin{aligned}
& \frac{\mathrm{d}}{\mathrm{d} t} \int_{\Omega} \frac{\left|\nabla w_{\epsilon}\right|^{2}}{w_{\epsilon}}+\left(k_{1}-\delta_{1}\right) \int_{\Omega} w_{\epsilon}\left|D^{2} \ln w_{\epsilon}\right|^{2}+\left(\frac{k_{1}}{2}-\delta_{2}\right) \int_{\Omega} \frac{\left|\nabla w_{\epsilon}\right|^{4}}{w_{\epsilon}^{3}} \\
& \quad \leq-2 \gamma \int_{\Omega} \frac{\nabla c_{\epsilon} \cdot \nabla n_{\epsilon}}{1+\epsilon n_{\epsilon}}+2 \gamma \epsilon \int_{\Omega} \frac{\nabla\left(\Delta c_{\epsilon}\right) \cdot \nabla n_{\epsilon}}{1+\epsilon n_{\epsilon}}+k_{2} \int_{\Omega} w_{\epsilon}+C_{\delta_{1}, \delta_{2}} \int_{\Omega}\left|w_{\epsilon}\right|^{5} \\
& \quad+C_{\delta_{1}, \delta_{2}} \int_{\Omega}|g|^{5 / 2}+C k_{3} \int_{\Omega}\left|\nabla u_{\epsilon}\right|^{2},
\end{aligned}
$$

where we have used the uniform bound to $w_{\epsilon}$ provided by Lemma 3.6 (observe that, for $q>3, w_{0, \epsilon} \in W^{1, q}(\Omega) \hookrightarrow$ $\left.L^{\infty}(\Omega)\right)$.

Note that

$$
\begin{aligned}
2 \gamma \epsilon \int_{\Omega} \frac{\nabla\left(\Delta c_{\epsilon}\right) \cdot \nabla n_{\epsilon}}{1+\epsilon n_{\epsilon}} & =2 \gamma \epsilon^{1 / 2} \int_{\Omega} \frac{\nabla\left(\Delta c_{\epsilon}\right) \cdot \nabla n_{\epsilon}}{n_{\epsilon}^{1 / 2}} \frac{\left(\epsilon n_{\epsilon}\right)^{1 / 2}}{1+\epsilon n_{\epsilon}} \\
& \leq \delta_{3} \int_{\Omega} \frac{\left|\nabla n_{\epsilon}\right|^{2}}{n_{\epsilon}}+C_{\delta_{3}} \epsilon \gamma^{2} \int_{\Omega}\left|\nabla\left(\Delta c_{\epsilon}\right)\right|^{2}
\end{aligned}
$$

From (3.3) it holds that $\epsilon \nabla\left(\Delta c_{\epsilon}\right)=\nabla c_{\epsilon}-\nabla w_{\epsilon}$. Then, by replacing (3.35) in (3.34), we get 


$$
\begin{gathered}
\frac{\mathrm{d}}{\mathrm{d} t} \int_{\Omega} \frac{\left|\nabla w_{\epsilon}\right|^{2}}{w_{\epsilon}}+\left(k_{1}-\delta_{1}\right) \int_{\Omega} w_{\epsilon}\left|D^{2} \ln w_{\epsilon}\right|^{2}+\left(\frac{k_{1}}{2}-\delta_{2}\right) \int_{\Omega} \frac{\left|\nabla w_{\epsilon}\right|^{4}}{w_{\epsilon}^{3}} \\
\quad \leq-2 \gamma \int_{\Omega} \frac{\nabla c_{\epsilon} \cdot \nabla n_{\epsilon}}{1+\epsilon n_{\epsilon}}+\delta_{3} \int_{\Omega} \frac{\left|\nabla n_{\epsilon}\right|^{2}}{n_{\epsilon}}+k_{2} \int_{\Omega} w_{\epsilon}+C_{\delta_{1}, \delta_{2}} \int_{\Omega}\left|w_{\epsilon}\right|^{5} \\
\quad+C_{\delta_{1}, \delta_{2}} \int_{\Omega}|g|^{5 / 2}+C k_{3} \int_{\Omega}\left|\nabla u_{\epsilon}\right|^{2}+\int_{\Omega}\left|\nabla c_{\epsilon}\right|^{2}+\int_{\Omega}\left|\nabla w_{\epsilon}\right|^{2} .
\end{gathered}
$$

Now, for the velocity $u_{\epsilon}$ we have the following estimate: there exist $\delta_{3}, C>0$ such that for every $\epsilon>0$

$$
\frac{1}{2} \frac{\mathrm{d}}{\mathrm{d} t} \int_{\Omega}\left|u_{\epsilon}\right|^{2}+\left(1-\delta_{3}\right) \int_{\Omega}\left|\nabla u_{\epsilon}\right|^{2} \leq C_{\delta_{3}} \int_{\Omega} \frac{\left|\nabla n_{\epsilon}\right|^{2}}{n_{\epsilon}}+C \int_{\Omega}|f|^{2}+C, \quad \text { on }(0, T) .
$$

Indeed, multiplying $(3.2)_{3}$ by $u_{\epsilon}$, and integrating in $\Omega$

$$
\frac{1}{2} \frac{\mathrm{d}}{\mathrm{d} t}\left\|u_{\epsilon}\right\|_{L^{2}}^{2}+\left\|\nabla u_{\epsilon}\right\|_{L^{2}}^{2}=\left(n_{\epsilon} \nabla \Phi, u_{\epsilon}\right)+\left(f, u_{\epsilon}\right)
$$

Moreover, since $n_{\epsilon} \in L^{1}(\Omega)$, by the Gagliardo-Niremberg inequality

$$
\begin{aligned}
\left(n_{\epsilon} \nabla \Phi, u_{\epsilon}\right) & \leq C\left\|n_{\epsilon}\right\|_{L^{6 / 5}}\left\|u_{\epsilon}\right\|_{L^{6}} \\
& \leq C\left\|n_{\epsilon}^{1 / 2}\right\|_{L^{12 / 5}}^{2}\left\|\nabla u_{\epsilon}\right\|_{L^{2}} \\
& \leq \delta_{3}\left\|\nabla u_{\epsilon}\right\|_{L^{2}}^{2}+C_{\delta_{3}}\left\|n_{\epsilon}^{1 / 2}\right\|_{L^{6 / 5}}^{4} \\
& \leq \delta_{3}\left\|\nabla u_{\epsilon}\right\|_{L^{2}}^{2}+C_{\delta_{3}}\left(\left\|n_{\epsilon}^{1 / 2}\right\|_{L^{2}}^{3 / 2}\left\|\nabla n_{\epsilon}^{1 / 2}\right\|_{L^{2}}^{1 / 2}+\left\|n_{\epsilon}^{1 / 2}\right\|_{L^{2}}^{2}\right)^{2} \\
& \leq \delta_{3}\left\|\nabla u_{\epsilon}\right\|_{L^{2}}^{2}+C_{\delta_{3}}\left\|n_{\epsilon}\right\|_{L^{1}}\left(\left\|n_{\epsilon}\right\|_{L^{1}}^{2}\left\|\nabla n_{\epsilon}^{1 / 2}\right\|_{L^{2}}^{1 / 2}+1\right)^{2} \\
& \leq \delta_{3}\left\|\nabla u_{\epsilon}\right\|_{L^{2}}^{2}+C_{\delta_{3}}\left(\left\|\nabla n_{\epsilon}^{1 / 2}\right\|_{L^{2}}+1\right) .
\end{aligned}
$$

Then, multiplying (3.29) by a suitable constant to cancel terms with (3.36), and multiplying (3.37) by a constant if necessary, we obtain

$$
\begin{aligned}
\frac{\mathrm{d}}{\mathrm{d} t}\left[\int_{\Omega} n_{\epsilon} \ln n_{\epsilon}+C \int_{\Omega} \frac{\left|\nabla w_{\epsilon}\right|^{2}}{w_{\epsilon}}\right. & \left.+C \int_{\Omega}\left|u_{\epsilon}\right|^{2}\right]+C \int_{\Omega} \frac{\left|\nabla n_{\epsilon}\right|^{2}}{n_{\epsilon}} \\
& +C \int_{\Omega} w_{\epsilon}\left|D^{2} \ln w_{\epsilon}\right|^{2}+C \int_{\Omega} \frac{\left|\nabla w_{\epsilon}\right|^{4}}{w_{\epsilon}^{3}}+C \int_{\Omega}\left|\nabla u_{\epsilon}\right|^{2} \\
& \leq C \int_{\Omega} w_{\epsilon}+C \int_{\Omega}\left|w_{\epsilon}\right|^{5}+C \int_{\Omega}|g|^{5 / 2}+C \int_{\Omega}|f|^{2}+\int_{\Omega}\left|\nabla c_{\epsilon}\right|^{2}+\int_{\Omega}\left|\nabla w_{\epsilon}\right|^{2} .
\end{aligned}
$$

Finally, by using Lemma 3.6, we get

$$
\begin{aligned}
& \frac{\mathrm{d}}{\mathrm{d} t}\left[\int_{\Omega} n_{\epsilon} \ln n_{\epsilon}+C \int_{\Omega} \frac{\left|\nabla w_{\epsilon}\right|^{2}}{w_{\epsilon}}+C \int_{\Omega}\left|u_{\epsilon}\right|^{2}\right]+C \int_{\Omega} \frac{\left|\nabla n_{\epsilon}\right|^{2}}{n_{\epsilon}} \\
& \quad+C \int_{\Omega} w_{\epsilon}\left|D^{2} \ln w_{\epsilon}\right|^{2}+C \int_{\Omega} \frac{\left|\nabla w_{\epsilon}\right|^{4}}{w_{\epsilon}^{3}}+C \int_{\Omega}\left|\nabla u_{\epsilon}\right|^{2} \\
& \quad \leq C \int_{\Omega}|g|^{5 / 2}+C \int_{\Omega}|f|^{2}+\int_{\Omega}\left|\nabla c_{\epsilon}\right|^{2}+\int_{\Omega}\left|\nabla w_{\epsilon}\right|^{2}+C .
\end{aligned}
$$


Testing (3.3) by $c_{\epsilon}$ and $-\Delta c_{\epsilon}$ give us

$$
\frac{1}{2}\left\|c_{\epsilon}\right\|_{L^{2}}^{2}+\epsilon\left\|\nabla c_{\epsilon}\right\|_{L^{2}}^{2} \leq \frac{1}{2}\left\|w_{\epsilon}\right\|_{L^{2}}^{2}
$$

and

$$
\frac{1}{2}\left\|\nabla c_{\epsilon}\right\|_{L^{2}}^{2}+\epsilon\left\|\Delta c_{\epsilon}\right\|_{L^{2}}^{2} \leq \frac{1}{2}\left\|\nabla w_{\epsilon}\right\|_{L^{2}}^{2},
$$

accordingly. Recalling that $\left\|\nabla w_{\epsilon}\right\|_{L^{2}\left(L^{2}\right)} \leq C$ uniformly in $\epsilon$ (see (3.28)), then, by (3.41) we have $\left\|\nabla c_{\epsilon}\right\|_{L^{2}\left(L^{2}\right)} \leq C$ uniformly in $\epsilon$. Moreover, $\sqrt{\epsilon} \Delta c_{\epsilon}$ is bounded in $L^{2}\left(L^{2}\right)$ uniformly in $\epsilon$. Thus, integrating in time (3.39), in particular, we obtain that

$$
\left\|\frac{\nabla w_{\epsilon}}{w_{\epsilon}^{1 / 2}}\right\|_{L^{\infty}\left(L^{2}\right)} \leq C
$$

and since $\int_{\Omega}\left|\nabla w_{\epsilon}\right|^{2}=\int_{\Omega} \frac{\left|\nabla w_{\epsilon}\right|^{2}}{w_{\epsilon}} w_{\epsilon}$ and $w_{\epsilon}$ is uniformly bounded (Lem. 3.6), we get

$$
\left\|\nabla w_{\epsilon}\right\|_{L^{\infty}\left(L^{2}\right)} \leq C
$$

uniformly in $\epsilon$. Also, from (3.39), Lemma 3.6 and the identity $\Delta w_{\epsilon}=w_{\epsilon} \Delta \ln w_{\epsilon}+\frac{\left|\nabla w_{\epsilon}\right|^{2}}{w_{\epsilon}}$, we have

$$
\left\|\Delta w_{\epsilon}\right\|_{L^{2}\left(L^{2}\right)} \leq C
$$

uniformly in $\epsilon$. In addition, from (3.40)-(3.42) we have

$$
\left\{c_{\epsilon}\right\} \text { is bounded in } L^{\infty}\left(H^{1}\right),\left\{\sqrt{\epsilon} \Delta c_{\epsilon}\right\} \text { is bounded in } L^{\infty}\left(L^{2}\right) .
$$

Moreover, taking $\Delta$ in (3.3), and testing by $\Delta c_{\epsilon}$, give us

$$
\frac{1}{2}\left\|\Delta c_{\epsilon}\right\|_{L^{2}}^{2}+\epsilon\left\|\nabla \Delta c_{\epsilon}\right\|_{L^{2}}^{2} \leq \frac{1}{2}\left\|\Delta w_{\epsilon}\right\|_{L^{2}}^{2}
$$

Therefore, by (3.43)

$$
\left\{c_{\epsilon}\right\} \text { is bounded in } L^{2}\left(H^{2}\right),\left\{\sqrt{\epsilon} \Delta c_{\epsilon}\right\} \text { is bounded in } L^{2}\left(H^{1}\right) .
$$

Thus we have

$$
\left\{\begin{array}{l}
\left\{c_{\epsilon}\right\} \text { is bounded in } L^{\infty}\left(H^{1}\right) \cap L^{2}\left(H^{2}\right) \hookrightarrow L^{10}\left(L^{10}\right), \\
\left\{\sqrt{\epsilon} \Delta c_{\epsilon}\right\} \text { is bounded in } L^{\infty}\left(L^{2}\right) \cap L^{2}\left(H^{1}\right) .
\end{array}\right.
$$

Since $2 \nabla \sqrt{n_{\epsilon}}=\frac{\nabla n_{\epsilon}}{\sqrt{n_{\epsilon}}}$, by (3.39), $\nabla \sqrt{n_{\epsilon}} \in L^{2}\left(L^{2}\right)$. Also $\sqrt{n_{\epsilon}} \in L^{2}\left(H^{1}\right) \hookrightarrow L^{2}\left(L^{6}\right)$. Moreover, by the mass conservation property $n_{\epsilon} \in L^{\infty}\left(L^{1}\right)$, which implies $\sqrt{n_{\epsilon}} \in L^{\infty}\left(L^{2}\right)$. That is,

$$
\left\{\begin{array}{l}
\left\{\sqrt{n_{\epsilon}}\right\} \text { is bounded in } L^{\infty}\left(L^{2}\right) \cap L^{2}\left(L^{6}\right) \hookrightarrow L^{10 / 3}\left(L^{10 / 3}\right), \\
\left\{\nabla \sqrt{n_{\epsilon}}\right\} \text { is bounded in } L^{2}\left(L^{2}\right) .
\end{array}\right.
$$


Since $\nabla n_{\epsilon}=2 \sqrt{n_{\epsilon}} \nabla \sqrt{n_{\epsilon}}$, by (3.45) we have

$$
\left\{n_{\epsilon}\right\} \text { is bounded in } L^{5 / 4}\left(W^{1,5 / 4}\right) \text {. }
$$

Moreover, by $(3.45)_{1}$ it holds

$$
\left\{n_{\epsilon}\right\} \text { is bounded in } L^{5 / 3}\left(L^{5 / 3}\right) \text {. }
$$

Also, from (3.39) we get

$$
\left\{u_{\epsilon}\right\} \text { is bounded in } L^{\infty}\left(L^{2}\right) \cap L^{2}(V) \hookrightarrow L^{10 / 3}\left(L^{10 / 3}\right),
$$

and from (3.42) and (3.43) we obtain

$$
\left\{w_{\epsilon}\right\} \text { is bounded in } L^{\infty}\left(H^{1}\right) \cap L^{2}\left(H^{2}\right) \hookrightarrow L^{10}\left(L^{10}\right) .
$$

\subsection{The limit as $\epsilon \rightarrow 0$}

Notice that from (3.3) and $(3.44)_{2}$ we obtain

$$
w_{\epsilon}-c_{\epsilon}=-\epsilon \Delta c_{\epsilon} \rightarrow 0 \quad \text { as } \epsilon \rightarrow 0, \quad \text { in } L^{\infty}\left(L^{2}\right) \cap L^{2}\left(H^{1}\right) .
$$

Therefore, from $(3.44)_{1}$ and $(3.45)-(3.50)$, there exist limit functions $[n, w, u]$ such that

$$
\left\{\begin{array}{l}
n \in L^{5 / 3}\left(L^{5 / 3}\right) \cap L^{5 / 4}\left(W^{1,5 / 4}\right), \\
c \in L^{\infty}\left(H^{1}\right) \cap L^{2}\left(H^{2}\right), \\
u \in L^{\infty}\left(L^{2}\right) \cap L^{2}(V),
\end{array}\right.
$$

and for some subsequence of $\left\{\left[n_{\epsilon}, c_{\epsilon}, u_{\epsilon}, w_{\epsilon}\right]\right\}_{\epsilon>0}$, denoted in the same way, the following convergences hold, as $\epsilon \rightarrow 0$,

$$
\left\{\begin{array}{l}
n_{\epsilon} \rightarrow n \quad \text { weakly in } L^{5 / 3}\left(L^{5 / 3}\right) \cap L^{5 / 4}\left(W^{1,5 / 4}\right), \\
c_{\epsilon} \rightarrow c \quad \text { weakly in } L^{2}\left(H^{2}\right) \text { and weakly* in } L^{\infty}\left(H^{1}\right), \\
u_{\epsilon} \rightarrow u \quad \text { weakly in } L^{2}(V) \text { and weakly* in } L^{\infty}\left(L^{2}\right), \\
w_{\epsilon} \rightarrow c \quad \text { weakly in } L^{2}\left(H^{1}\right) \text { and weakly* in } L^{\infty}\left(L^{2}\right) .
\end{array}\right.
$$

Now, by $(3.44)_{1},\left\{\nabla c_{\epsilon}\right\}$ is bounded in $L^{\infty}\left(L^{2}\right) \cap L^{2}\left(H^{1}\right) \hookrightarrow L^{10 / 3}\left(L^{10 / 3}\right)$. Thus, from (3.47) we have

$$
\left\{\frac{n_{\epsilon}^{+}}{1+\epsilon n_{\epsilon}^{+}} \nabla c_{\epsilon}\right\} \text { is bounded in } L^{10 / 9}\left(L^{10 / 9}\right)
$$

which implies the term $\chi \int_{0}^{T} \int_{\Omega} \frac{n_{\epsilon}^{+}}{1+\epsilon n_{\epsilon}^{+}} \nabla c_{\epsilon} \cdot \nabla \varphi$ is bounded for each $\varphi \in L^{10}\left(W^{1,10}\right)$. Likewise, by (3.47) and (3.48) we get

$$
\left\{n_{\epsilon} u_{\epsilon}\right\} \text { is bounded in } L^{10 / 9}\left(L^{10 / 9}\right) \text {. }
$$


Therefore, looking for the $n_{\epsilon}$-equation in (3.2) we obtain

$$
\left\{\partial_{t} n_{\epsilon}\right\} \text { is bounded in } L^{10 / 9}\left(\left(W^{1,10}\right)^{\prime}\right) .
$$

On the other hand, from (3.48) and Lemma 3.6 we get

$$
\left\{u_{\epsilon} w_{\epsilon}\right\} \text { is bounded in } L^{10 / 3}\left(L^{10 / 3}\right) \text {, }
$$

which implies $\int_{0}^{T} \int_{\Omega} w_{\epsilon} u_{\epsilon} \cdot \nabla \varphi$ is bounded for $\varphi \in L^{5 / 3}\left(W^{1,5 / 3}\right)$. Also, from Lemma 3.6 it holds

$$
\begin{aligned}
\left\{g w_{\epsilon}\right\} & \text { is bounded in } L^{5 / 2}\left(L^{5 / 2}\right), \\
\left\{w_{\epsilon}^{+} \frac{\ln \left(1+\epsilon n_{\epsilon}^{+}\right)}{\epsilon}\right\} & \text { is bounded in } L^{5 / 3}\left(L^{5 / 3}\right) .
\end{aligned}
$$

Therefore, looking for the $w_{\epsilon}$-equation in (3.2) we obtain

$$
\left\{\partial_{t} w_{\epsilon}\right\} \text { is bounded in } L^{5 / 3}\left(L^{5 / 3}\right)
$$

Now, for $\psi \in L^{5 / 2}\left(W^{1,5 / 2}\right)$, from (3.47), (3.48), we obtain

$$
\begin{aligned}
& \left(u_{\epsilon} \otimes u_{\epsilon}, \nabla \psi\right) \leq\left\|u_{\epsilon}^{2}\right\|_{L^{5 / 3}}\|\nabla \psi\|_{L^{5 / 2}}, \\
& \left(n_{\epsilon} \nabla \Phi, \psi\right) \leq\|\nabla \Phi\|_{\infty}\left\|n_{\epsilon}\right\|_{L^{5 / 3}}\|\psi\|_{L^{5 / 2}} .
\end{aligned}
$$

Therefore, looking for the $u_{\epsilon}$-equation in (3.2) we obtain

$$
\left\{\partial_{t} u_{\epsilon}\right\} \text { is bounded in } L^{5 / 3}\left(\left(W^{1,5 / 3}\right)^{\prime}\right) .
$$

Therefore, from the time-estimates above we have

$$
\begin{cases}\partial_{t} n_{\epsilon} \rightarrow \partial_{t} n & \text { weakly in } L^{10 / 9}\left(\left(W^{1,10}\right)^{\prime}\right) \\ \partial_{t} w_{\epsilon} \rightarrow \partial_{t} c & \text { weakly in } L^{5 / 3}\left(L^{5 / 3}\right) \\ \partial_{t} u_{\epsilon} \rightarrow \partial_{t} u & \text { weakly in } L^{5 / 3}\left(\left(W^{1,3 / 2}\right)^{\prime}\right)\end{cases}
$$

From (3.45), (3.46), (3.48), (3.49), (3.62), taking into account that $W^{1,5 / 4} \stackrel{c}{\hookrightarrow} L^{2} \hookrightarrow\left(W^{1,10}\right)^{\prime}, H^{2} \stackrel{c}{\hookrightarrow} H^{1} \hookrightarrow$ $L^{5 / 3}$ and $H^{1} \stackrel{c}{\hookrightarrow} L^{2} \hookrightarrow L^{5 / 3}$, and using the Aubin-Lions lemma and the Simon compactness theorem, we have

$$
\begin{cases}n_{\epsilon} \rightarrow n & \text { strongly in } L^{5 / 4}\left(L^{2}\right) \cap C\left([0, T] ;\left(W^{1,10}\right)^{\prime}\right) \\ w_{\epsilon} \rightarrow c & \text { strongly in } L^{2}\left(H^{1}\right) \cap C\left([0, T] ; L^{2}\right) \\ u_{\epsilon} \rightarrow u & \text { strongly in } L^{2}\left(L^{2}\right)\end{cases}
$$

By (3.52), we have the convergence

$$
\frac{n_{\epsilon}^{+}}{1+\epsilon n_{\epsilon}^{+}} \nabla c_{\epsilon} \rightarrow \zeta \quad \text { weakly in } L^{10 / 9}\left(L^{10 / 9}\right) .
$$


From $(3.51)_{2}$ we have $\nabla c_{\epsilon} \rightarrow \nabla c$ weakly in $L^{10 / 3}\left(L^{10 / 3}\right)$. Also from $(3.63)_{1},\left\{n_{\epsilon}\right\}_{\epsilon>0}$ is relatively compact in $L^{5 / 4}\left(L^{5 / 4}\right)$. Then, taking into account (3.47), and the interpolation inequality

$$
\left\|n_{\epsilon}\right\|_{L^{p}\left(L^{p}\right)} \leq\left\|n_{\epsilon}\right\|_{L^{5 / 4}\left(L^{5 / 4}\right)}^{1-\theta}\left\|n_{\epsilon}\right\|_{L^{5 / 3}\left(L^{5 / 3}\right)}^{\theta}, \theta=\frac{4 p-5}{p} \in(0,1),
$$

(which leads to $\frac{5}{4}<p<\frac{5}{3}$ ), we obtain that $\left\{n_{\epsilon}\right\}$ is relatively compact in $L^{p}\left(L^{p}\right)$. Since $L^{5 / 3}\left(L^{5 / 3}\right) \hookrightarrow$ $L^{20 / 13}\left(L^{20 / 13}\right)$ and noting that

$$
\begin{aligned}
\int_{\Omega}\left(n_{\epsilon}^{+} \nabla c_{\epsilon}-n^{+} \nabla c\right) \varphi & =\int_{\Omega} n_{\epsilon}^{+}\left(\nabla c_{\epsilon}-\nabla c\right) \varphi+\int_{\Omega}\left(n_{\epsilon}^{+}-n^{+}\right) \nabla c_{\epsilon} \varphi \\
& \leq \int_{\Omega} n_{\epsilon}^{+}\left(\nabla c_{\epsilon}-\nabla c\right) \varphi+\left\|n_{\epsilon}^{+}-n^{+}\right\|_{L^{20 / 13}}\left\|\nabla c_{\epsilon}\right\|_{L^{10 / 3}}\|\varphi\|_{L^{20}} \\
& \underset{\epsilon \rightarrow 0}{\longrightarrow} 0
\end{aligned}
$$

we can conclude, from (3.64), that $\zeta=n^{+} \nabla c$. That is

$$
\frac{n_{\epsilon}^{+}}{1+\epsilon n_{\epsilon}^{+}} \nabla c_{\epsilon} \rightarrow n^{+} \nabla c \quad \text { weakly in } L^{10 / 9}\left(L^{10 / 9}\right) .
$$

In a similar way, by $(3.51)_{3},(3.53)$ and using that $\left\{n_{\epsilon}\right\}_{\epsilon>0}$ is relatively compact in $L^{p}\left(L^{p}\right)$ for all $p<5 / 3$, we get

$$
n_{\epsilon} u_{\epsilon} \rightarrow n u \quad \text { weakly in } L^{10 / 9}\left(L^{10 / 9}\right) .
$$

Therefore, taking the limit in the regularized problem $(3.2)_{1}$, as $\epsilon \rightarrow 0$, by $(3.51)_{1},(3.65)$ and (3.66), the limit $n$ satisfies the weak formulation

$$
\int_{0}^{T}\left\langle\partial_{t} n, \varphi\right\rangle+\int_{0}^{T} \int_{\Omega} \nabla n \cdot \nabla \varphi-\int_{0}^{T} \int_{\Omega} n u \cdot \nabla \varphi-\chi \int_{0}^{T} \int_{\Omega} n^{+} \nabla c \cdot \nabla \varphi=0, \quad \forall \varphi \in L^{10}\left(W^{1,10}\right) .
$$

On the other hand, from (3.51), (3.55) and $(3.63)_{2}$ we get

$$
u_{\epsilon} w_{\epsilon} \rightarrow c u \quad \text { weakly in } L^{5 / 3}\left(L^{5 / 3}\right) .
$$

Also, from $(3.63)_{2}$ and taking into account that $g \in L^{5 / 2}\left(L^{5 / 2}\right)$, in particular, it holds

$$
g w_{\epsilon}^{+} \rightarrow g c^{+} \quad \text { weakly in } L^{10 / 7}\left(L^{10 / 7}\right) \text {. }
$$

Now, since $\frac{\ln \left(1+\epsilon n_{\epsilon}^{+}\right)}{\epsilon} \leq n_{\epsilon}^{+}$, by (3.47) and (3.49) we have

$$
\left\{w_{\epsilon}^{+} \frac{\ln \left(1+\epsilon n_{\epsilon}^{+}\right)}{\epsilon}\right\} \text { is bounded in } L^{10 / 7}\left(L^{10 / 7}\right) .
$$

By (3.47) and the dominated convergence theorem

$$
\left\|\frac{\ln \left(1+\epsilon n_{\epsilon}^{+}\right)}{\epsilon}-n^{+}\right\|_{L^{5 / 3}\left(L^{5 / 3}\right)}
$$




$$
\begin{aligned}
& \leq\left\|\frac{\ln \left(1+\epsilon n_{\epsilon}^{+}\right)}{\epsilon}-\frac{\ln \left(1+\epsilon n^{+}\right)}{\epsilon}\right\|_{L^{5 / 3}\left(L^{5 / 3}\right)}+\left\|\frac{\ln \left(1+\epsilon n^{+}\right)}{\epsilon}-n^{+}\right\|_{L^{5 / 3}\left(L^{5 / 3}\right)} \\
& =\frac{1}{\epsilon}\left\|\ln \left(1+\frac{\epsilon\left(n_{\epsilon}^{+}-n^{+}\right)}{1+\epsilon n^{+}}\right)\right\|_{L^{5 / 3}\left(L^{5 / 3}\right)}+\left\|\frac{\ln \left(1+\epsilon n^{+}\right)}{\epsilon}-n^{+}\right\|_{L^{5 / 3}\left(L^{5 / 3}\right)} \\
& \underset{\epsilon \rightarrow 0}{\longrightarrow} 0 .
\end{aligned}
$$

Then, by the weak convergence in $(3.51)_{4}$, the strong convergence above, and (3.70), we get

$$
w_{\epsilon}^{+} \frac{\ln \left(1+\epsilon n_{\epsilon}^{+}\right)}{\epsilon} \rightarrow c^{+} n^{+} \quad \text { weakly in } L^{10 / 7}\left(L^{10 / 7}\right) .
$$

Therefore, from (3.62), (3.63), (3.68), (3.69) and (3.71) we get

$$
\int_{0}^{T}\left\langle\partial_{t} c, \varphi\right\rangle+\int_{0}^{T} \int_{\Omega} \nabla c \cdot \nabla \varphi-\int_{0}^{T} \int_{\Omega} c u \cdot \nabla \varphi-\gamma \int_{0}^{T} \int_{\Omega} c^{+} n^{+} \varphi-\int_{0}^{T} \int_{\Omega} g c^{+} \varphi=0,
$$

for all $\varphi \in L^{10 / 3}\left(W^{1,10 / 3}\right)$. Observe that (3.72) is a weak formulation for the $c$-equation of (1.2). Thus, integrating by parts in (3.72) and using that $n \in L^{5 / 3}\left(L^{5 / 3}\right), c \in L^{2}\left(H^{2}\right) \cap L^{\infty}\left(H^{1}\right)$, and $u \in L^{2}\left(H^{1}\right) \cap L^{\infty}\left(L^{2}\right)$, we get

$$
c_{t}+u \cdot \nabla c=\Delta c-\gamma c n+g c, \quad \text { in } L^{10 / 7}\left(L^{10 / 7}\right) .
$$

On the other hand, to take the limit in the $u_{\epsilon}$-equation, we argue as usual. In fact, recalling (3.18), $\left\{Y_{\epsilon} u_{\epsilon}\right\}$ is uniformly bounded in $L^{2}\left(L^{6}\right)$. Moreover,

$$
Y_{\epsilon} u_{\epsilon} \otimes u_{\epsilon}-u \otimes u=Y_{\epsilon} u_{\epsilon} \otimes\left(u_{\epsilon}-u\right)+Y_{\epsilon}\left(u_{\epsilon}-u\right) \otimes u_{\epsilon}+\left(Y_{\epsilon}-I\right) u \otimes u .
$$

Therefore, taking into account the above decomposition, $(3.63)_{3}$ and the fact that $Y_{\epsilon} \rightarrow I$ in $L^{2}$, we can pass to the limit in the $u_{\epsilon}$-equation in order to get

$$
\int_{0}^{T}\left\langle\partial_{t} u, \psi\right\rangle+\int_{0}^{T} \int_{\Omega} \nabla u \cdot \nabla \psi-\int_{0}^{T} \int_{\Omega} u \otimes u \cdot \nabla \psi=\int_{0}^{T} \int_{\Omega} n \nabla \Phi \cdot \psi+\int_{0}^{T} \int_{\Omega} f \cdot \psi
$$

for all $\psi \in L^{5 / 2}\left(W^{1,5 / 2}\right)$.

Finally, from (3.4), $\left[n_{\epsilon}(x, 0), w_{\epsilon}(x, 0), u_{\epsilon}(x, 0)\right]=\left[n_{0, \epsilon}(x), w_{0, \epsilon}(x), u_{0, \epsilon}(x)\right] \rightarrow\left[n_{0}, c_{0}, u_{0}\right]$ as $\epsilon \rightarrow 0$ and thus, by (3.63), $n(0)=n_{0}, c(0)=c_{0}, u(0)=u_{0}$, which is the initial condition in (1.3).

To conclude the proof of Theorem 3.2 it remains to see the non-negativity of $n$ and $c$. From (3.63) and taking into account that $n_{\epsilon}, c_{\epsilon} \geq 0$ a.e. $(x, t) \in \Omega \times(0, T)$, for all $\epsilon>0$, then $n, c \geq 0$.

\section{REgUlARITY CRITERION}

In this section we are going to prove that under the extra assumption $[n, u] \in L^{20 / 7}\left(L^{20 / 7}\right) \times L^{8}\left(L^{4}\right)$, weak solutions have better regularity properties. This is possible by a bootstrapping argument. It is worth noting that this extra regularity assumption is going to be obtained from the definition of the functional in the control problem, providing the relation between the solutions of the PDE, the cost functional and the existence of optimal solutions. 
Definition 4.1. (Strong solution of (1.2)) Let $g \in L^{4}\left(L^{4}\right), f \in L^{2}\left(L^{2}\right), \nabla \Phi \in L^{\infty}(\Omega),\left[n_{0}, c_{0}, u_{0}\right] \in \widehat{W}^{3 / 2,4} \times$ $\widehat{W}^{3 / 2,4} \times V, n_{0} \geq 0, c_{0} \geq 0$ in $\Omega$. A strong solution of (1.2)-(1.3) is a triple $[n, c, u]$ of functions such that

$$
[n, c, u] \in \mathcal{X}_{4} \times \mathcal{X}_{4} \times \mathcal{X}_{2},
$$

such that (1.2) holds a.e $(x, t)$ in $\Omega \times(0, T)$, and (1.3) are satisfied.

Theorem 4.2. (Regularity criterion) Let $[n, c, u]$ be a weak solution of $(1.2)$. If $\left[n_{0}, c_{0}, u_{0}\right] \in \widehat{W}^{3 / 2,4} \times \widehat{W}^{3 / 2,4} \times$ $V$ and

$$
[n, u] \in L^{20 / 7}\left(L^{20 / 7}\right) \times L^{8}\left(L^{4}\right),
$$

then $[n, c, u]$ is a strong solution of (1.2) in the sense of Definition 4.1. Moreover, there is a constant $C=$ $C\left(\left\|n_{0}\right\|_{\widehat{W}^{3 / 2,4}},\left\|c_{0}\right\|_{\widehat{W}^{3 / 2,4}},\left\|u_{0}\right\|_{V}, g \in L^{4}\left(L^{4}\right),\|f\|_{L^{2}\left(L^{2}\right)},\|n\|_{L^{2}\left(L^{2}\right)},\|u\|_{L^{8}\left(L^{4}\right)}\right)>0$, such that

$$
\|[n, c, u]\|_{\mathcal{X}_{4} \times \mathcal{X}_{4} \times \mathcal{X}_{2}} \leq C .
$$

Proof. The idea is to use a bootstrapping argument following the ideas of [25]. From Theorem 3.2, there exists a weak solution $[n, c, u]$ of system (1.2) in the sense of Definition 3.1. The regularity is carried out into several steps:

Step 1: $u \in \mathcal{X}_{2}$.

Since $u \in L^{8}\left(L^{4}\right)$, from Theorem 4.2.1 in [5], we have $u \in \mathcal{X}_{2} \hookrightarrow L^{10}\left(L^{10}\right)$. The last embedding is consequence of Lemma A.4 below, applied to $q_{1}=6, p_{1}=p_{2}=2, r=2$. Moreover,

$$
\|u\|_{\mathcal{X}_{2}} \leq C\left(\left\|u_{0}\right\|_{V}+\|\Phi\|_{L^{\infty}}\|n\|_{L^{2}\left(L^{2}\right)}+\|u\|_{L^{8}\left(L^{4}\right)}\right) .
$$

Step 2: $c \in \mathcal{X}_{20 / 7}$.

As a general fact in the following steps, $n \in L^{20 / 7}\left(L^{20 / 7}\right)$ and $u \in L^{10}\left(L^{10}\right)$. Moreover, from the weak regularity we have $c \in \mathcal{X}_{2} \hookrightarrow L^{10}\left(L^{10}\right)$.

i) $c \in \mathcal{X}_{20 / 9}$.

From the corresponding regularities of $c, n, g$, we have $g c \in L^{20 / 7}\left(L^{20 / 7}\right)$ and $c n \in L^{20 / 9}\left(L^{20 / 9}\right)$. Moreover, since $c \in \mathcal{X}_{2}$, we have $\nabla c \in L^{\infty}\left(L^{2}\right) \cap L^{2}\left(H^{1}\right) \hookrightarrow L^{10 / 3}\left(L^{10 / 3}\right)$, where we have used Lemma A.4 with $q_{1}=p_{1}=p_{2}=2, r=1$. Therefore $u \cdot \nabla c \in L^{5 / 2}\left(L^{5 / 2}\right)$. In conclusion, from $(1.2)_{2}, c_{t}-\Delta c \in L^{20 / 9}\left(L^{20 / 9}\right)$, thus from Theorem 2.1, $c \in \mathcal{X}_{20 / 9}$.

ii) $c \in L^{20}\left(L^{20}\right)$.

Since $c \in \mathcal{X}_{20 / 9}$, we have $c \in L^{\infty}\left(W^{11 / 10,20 / 9}\right) \cap L^{20 / 9}\left(W^{2,20 / 9}\right) \hookrightarrow L^{\infty}\left(L^{12}\right) \cap L^{20 / 9}\left(W^{2,20 / 9}\right)$. Thus, from Lemma A.4 with $q_{1}=12, p_{1}=p_{2}=20 / 9, r=2$, we obtain $c \in L^{20}\left(L^{20}\right)$.

iii) $c \in \mathcal{X}_{5 / 2}$.

With the extra regularity obtained for $c$, we have $c n \in L^{5 / 2}\left(L^{5 / 2}\right)$. Since $\frac{5}{2}<\frac{20}{7}$, from Theorem 2.1 , we have $c \in \mathcal{X}_{5 / 2}$.

iv) $c \in L^{\infty}\left(L^{\infty}\right)$ and $\nabla c \in L^{5}\left(L^{5}\right)$.

Since $c \in \mathcal{X}_{5 / 2}$, then $c \in L^{\infty}\left(W^{6 / 5,5 / 2}\right) \cap L^{5 / 2}\left(W^{2,5 / 2}\right)$. Now, by Sobolev embeddings $W^{6 / 5,5 / 2}(\Omega) \hookrightarrow$ $L^{q}(\Omega)$, for all $1 \leq q<\infty$. Thus $c \in L^{\infty}\left(L^{q}\right)$ for all $1 \leq q<\infty$; in particular, $c \in L^{\infty}\left(L^{260 / 9}\right)$ and then, $n c \in L^{20 / 7}\left(L^{13 / 5}\right)$. Thus, from Theorem 2.1 we get $c \in L^{13 / 5}\left(W^{2,13 / 5}\right) \cap L^{\infty}\left(W^{16 / 13,13 / 5}\right) \hookrightarrow L^{\infty}\left(L^{\infty}\right)$. Moreover $c \in \mathcal{X}_{5 / 2}$ implies $\nabla c \in L^{\infty}\left(W^{1 / 5,5 / 2}\right) \cap L^{5 / 2}\left(W^{1,5 / 2}\right) \hookrightarrow L^{\infty}\left(L^{3}\right) \cap L^{5 / 2}\left(W^{1,5 / 2}\right)$. Thus, from Lemma A.4 with $q_{1}=3, p_{1}=p_{2}=5 / 2, r=1$, we obtain $\nabla c \in L^{5}\left(L^{5}\right)$.

v) $c \in \mathcal{X}_{20 / 7}$.

With the extra regularity obtained for $c$ and $\nabla c$, we have that $c n \in L^{20 / 7}\left(L^{20 / 7}\right)$ and $u \cdot \nabla c \in L^{10 / 3}\left(L^{10 / 3}\right)$. Since $\frac{20}{7}<\frac{10}{3}$, from Theorem 2.1, $c \in \mathcal{X}_{20 / 7}$. 
vi) $\nabla c \in L^{20 / 3}\left(L^{20 / 3}\right)$ and $\Delta c \in L^{20 / 7}\left(L^{20 / 7}\right)$.

Since $c \in \mathcal{X}_{20 / 7}$, we have $\nabla c \in L^{\infty}\left(W^{3 / 10,20 / 7}\right) \cap L^{20 / 7}\left(W^{1,20 / 7}\right) \hookrightarrow L^{\infty}\left(L^{4}\right) \cap L^{20 / 7}\left(W^{1,20 / 7}\right)$. Thus, from Lemma A.4 with $q_{1}=4, p_{1}=p_{2}=20 / 7, r=1$, we obtain $\nabla c \in L^{20 / 3}\left(L^{20 / 3}\right)$. The fact that $\Delta c \in$ $L^{20 / 7}\left(L^{20 / 7}\right)$ is immediate from the definition of $\mathcal{X}_{20 / 7}$.

Notice that we cannot go further in the regularity of $c$ because $c n$ cannot be improved beyond $L^{20 / 7}\left(L^{20 / 7}\right)$. That is why we work now to improve the regularity of $n$, and go back later again on the regularity of $c$.

Step 3: $n \in L^{\infty}\left(L^{2}\right) \cap L^{2}\left(H^{1}\right)$.

First, from step 2-(vi) we have the following regularity property on $c$

$$
\nabla c \in L^{\infty}\left(L^{4}\right) \cap L^{20 / 7}\left(W^{1,20 / 7}\right) \hookrightarrow L^{20 / 3}\left(L^{20 / 3}\right) .
$$

Our starting point is the weak regularity, together with the extra regularity assumption of $n$; that is, $n \in$ $L^{20 / 7}\left(L^{20 / 7}\right) \cap L^{5 / 4}\left(W^{1,5 / 4}\right)$.

i) $n \in \mathcal{X}_{20 / 19}$.

Since $n, \Delta c \in L^{20 / 7}\left(L^{20 / 7}\right)$, we have $n \Delta c \in L^{10 / 7}\left(L^{10 / 7}\right)$. Moreover, $u \in L^{10}\left(L^{10}\right)$ and $\nabla n \in L^{5 / 4}\left(L^{5 / 4}\right)$ imply $u \cdot \nabla n \in L^{10 / 9}\left(L^{10 / 9}\right)$. Finally, $\nabla c \in L^{20 / 3}\left(L^{20 / 3}\right)$ and $\nabla n \in L^{5 / 4}\left(L^{5 / 4}\right)$ imply $\nabla c \cdot \nabla n \in$ $L^{20 / 19}\left(L^{20 / 19}\right)$. Since $\frac{20}{19}<\frac{10}{9}<\frac{10}{7}$, from $(1.2)_{1}, n_{t}-\Delta n \in L^{20 / 19}\left(L^{20 / 19}\right)$, thus from Theorem 2.1, $n \in \mathcal{X}_{20 / 19}$.

ii) $\nabla n \in L^{200 / 171}\left(L^{25 / 17}\right)$.

Since $n \in \mathcal{X}_{20 / 19}$, we have $n \in L^{\infty}\left(W^{1 / 10,20 / 19}\right) \cap L^{20 / 19}\left(W^{2,20 / 19}\right)$. Then, denoting by $D^{1 / 10} n$ the $1 / 10$ derivative of $u$ we have

$$
D^{1 / 10} n \in L^{\infty}\left(L^{20 / 19}\right) \cap L^{20 / 19}\left(W^{19 / 10,20 / 19}\right) \hookrightarrow L^{\infty}\left(L^{20 / 19}\right) \cap L^{20 / 19}\left(W^{1,20 / 13}\right) .
$$

Then, from Lemma A.5 with $\alpha=9 / 10, \beta=1, \gamma=0, p=20 / 13, q=20 / 19$, we have $\lambda=9 / 10, r=25 / 17$ and for $s>0$,

$$
\left\|D^{1 / 10} n\right\|_{W^{9 / 10,25 / 17}}^{s} \leq\left\|D^{1 / 10} n\right\|_{W^{1,20 / 13}}^{s \lambda}\left\|D^{1 / 10} n\right\|_{L^{20 / 19}}^{s(1-\lambda)} .
$$

Since $D^{1 / 10} n \in L^{20 / 19}$ in time, we have $s \lambda=20 / 19$, which implies $s=200 / 171$ and then $n \in$ $L^{200 / 171}\left(W^{1,25 / 17}\right)$. Finally,

$$
\nabla n \in L^{200 / 171}\left(L^{25 / 17}\right)
$$

iii) $n \in \mathcal{X}_{10 / 9}$.

From (4.4) we have $\nabla c \in L^{\infty}\left(L^{4}\right) \cap L^{20 / 7}\left(L^{60}\right)$. Moreover, from Lemma A.1 with $p_{1}=\infty, q_{1}=4, p_{2}=$ $20 / 7, q_{2}=60$ and $p=10$ we obtain

$$
\nabla c \in L^{\infty}\left(L^{4}\right) \cap L^{10}\left(L^{60 / 11}\right) .
$$

Therefore, from (4.5), $\nabla c \cdot \nabla n \in L^{200 / 171}\left(L^{100 / 93}\right) \cap L^{200 / 191}\left(L^{300 / 259}\right)$. Finally, from Lemma A.1 with $\left(p_{1}, q_{1}\right)=(200 / 171,100 / 93),\left(p_{2}, q_{2}\right)=(200 / 191,300 / 259)$ we have $\nabla c \cdot \nabla n \in L^{10 / 9}\left(L^{10 / 9}\right)$. From Theorem 2.1, $n \in \mathcal{X}_{10 / 9}$.

iv) $\nabla n \in L^{25 / 18}\left(L^{150 / 103}\right)$.

Since $n \in \mathcal{X}_{10 / 9}$, we have $n \in L^{\infty}\left(W^{1 / 5,10 / 9}\right) \cap L^{10 / 9}\left(W^{2,10 / 9}\right)$. Then,

$$
D^{1 / 5} n \in L^{\infty}\left(L^{10 / 9}\right) \cap L^{10 / 9}\left(W^{9 / 5,10 / 9}\right) \hookrightarrow L^{\infty}\left(L^{10 / 9}\right) \cap L^{10 / 9}\left(W^{1,30 / 19}\right) .
$$


Thus, from Lemma A.5 with $\alpha=4 / 5, \beta=1, \gamma=0, p=q=10 / 9$, we have $\lambda=4 / 5, r=150 / 103$ and for $s>0$,

$$
\left\|D^{1 / 5} n\right\|_{W^{4 / 5,150 / 103}}^{s} \leq\left\|D^{1 / 5} n\right\|_{W^{1,30 / 19}}^{s \lambda}\left\|D^{1 / 5} n\right\|_{L^{10 / 9}}^{s(-\lambda)}
$$

Since $D^{1 / 5} n \in L^{10 / 9}$ in time, we have $s \lambda=10 / 9$, which implies $s=25 / 18$ and then $n \in L^{25 / 18}\left(W^{1,150 / 103}\right)$. Finally,

$$
\nabla n \in L^{25 / 18}\left(L^{150 / 103}\right)
$$

v) $n \in \mathcal{X}_{20 / 17}$.

From (4.4) we know $\nabla c \in L^{\infty}\left(L^{4}\right) \cap L^{20 / 7}\left(L^{60}\right)$. Moreover, from Lemma A.1 with $p_{1}=\infty, q_{1}=4, p_{2}=$ $20 / 7, q_{2}=60$ and $p=5$ we obtain

$$
\nabla c \in L^{\infty}\left(L^{4}\right) \cap L^{5}\left(L^{60 / 7}\right)
$$

Therefore, from (4.6), $\nabla c \cdot \nabla n \in L^{25 / 18}\left(L^{300 / 281}\right) \cap L^{25 / 23}\left(L^{300 / 241}\right)$. Finally, from Lemma A.1 with $\left(p_{1}, q_{1}\right)=(25 / 18,300 / 281)$ and $\left(p_{2}, q_{2}\right)=(25 / 23,300 / 241)$ we have $\nabla c \cdot \nabla n \in L^{20 / 17}\left(L^{20 / 17}\right)$. From (4.6) we also have $u \cdot \nabla n \in L^{20 / 17}\left(L^{20 / 17}\right)$. Thus, we conclude, from Theorem 2.1, $n \in \mathcal{X}_{20 / 17}$.

vi) $\nabla n \in L^{200 / 119}\left(L^{150 / 103}\right)$.

Since $n \in \mathcal{X}_{20 / 17}$, we have $n \in L^{\infty}\left(W^{3 / 10,20 / 17}\right) \cap L^{20 / 17}\left(W^{2,20 / 17}\right)$. Then,

$$
D^{3 / 10} n \in L^{\infty}\left(L^{20 / 17}\right) \cap L^{20 / 17}\left(W^{17 / 10,20 / 17}\right) \hookrightarrow L^{\infty}\left(L^{20 / 17}\right) \cap L^{20 / 17}\left(W^{1,60 / 37}\right) .
$$

Thus, from Lemma A.5 with $\alpha=7 / 10, \beta=1, \gamma=0, p=60 / 37, q=20 / 17$, we have $\lambda=7 / 10, r=150 / 103$ and for $s>0$,

$$
\left\|D^{3 / 10} n\right\|_{W^{7 / 10,150 / 103}}^{s} \leq\left\|D^{3 / 10} n\right\|_{W^{1,60 / 37}}^{s \lambda}\left\|D^{3 / 10} n\right\|_{L^{20 / 17}}^{s(1-\lambda)}
$$

Since $D^{3 / 10} n \in L^{20 / 17}$ in time, we have $s \lambda=20 / 17$, which implies that $s=200 / 119$ and then $n \in$ $L^{200 / 119}\left(W^{1,150 / 103}\right)$. Finally,

$$
\nabla n \in L^{200 / 119}\left(L^{150 / 103}\right)
$$

vii) $n \in \mathcal{X}_{5 / 4}$.

From (4.4) we know $\nabla c \in L^{\infty}\left(L^{4}\right) \cap L^{20 / 7}\left(L^{60}\right)$. Moreover, from Lemma A.1 with $p_{1}=\infty, q_{1}=4, p_{2}=$ $20 / 7, q_{2}=60$ and $p=4$ we obtain

$$
\nabla c \in L^{\infty}\left(L^{4}\right) \cap L^{4}\left(L^{12}\right) .
$$

Therefore, from (4.7), $\nabla c \cdot \nabla n \in L^{200 / 119}\left(L^{300 / 281}\right) \cap L^{200 / 169}\left(L^{300 / 231}\right)$. Finally, from Lemma A.1 with $\left(p_{1}, q_{1}\right)=(200 / 119,300 / 281),\left(p_{2}, q_{2}\right)=(200 / 169,300 / 231)$ we have $\nabla c \cdot \nabla n \in L^{5 / 4}\left(L^{5 / 4}\right)$. In a similar way, from (4.7), we have $u \cdot \nabla n \in L^{5 / 4}\left(L^{5 / 4}\right)$. From Theorem 2.1 we conclude that $n \in \mathcal{X}_{5 / 4}$.

viii) $\nabla n \in L^{25 / 12}\left(L^{25 / 17}\right)$.

Since $n \in \mathcal{X}_{5 / 4}$, we have $n \in L^{\infty}\left(W^{2 / 5,5 / 4}\right) \cap L^{5 / 4}\left(W^{2,5 / 4}\right)$. Then,

$$
D^{2 / 5} n \in L^{\infty}\left(L^{5 / 4}\right) \cap L^{5 / 4}\left(W^{8 / 5,5 / 4}\right) \hookrightarrow L^{\infty}\left(L^{5 / 4}\right) \cap L^{5 / 4}\left(W^{1,5 / 3}\right) .
$$


Thus, from Lemma A.5 with $\alpha=3 / 5, \beta=1, \gamma=0, p=5 / 3, q=5 / 4$, we have $\lambda=3 / 5, r=25 / 17$ and for $s>0$,

$$
\left\|D^{2 / 5} n\right\|_{W^{3 / 5,25 / 17}}^{s} \leq\left\|D^{2 / 5} n\right\|_{W^{1,5 / 3}}^{s \lambda}\left\|D^{2 / 5} n\right\|_{L^{5 / 4}}^{s(1-\lambda)} .
$$

Since $D^{2 / 5} n \in L^{5 / 4}$ in time, we have $s \lambda=5 / 4$, which implies $s=25 / 12$ and then $n \in L^{25 / 12}\left(W^{1,25 / 17}\right)$. Finally,

$$
\nabla n \in L^{25 / 12}\left(L^{25 / 17}\right)
$$

ix) $n \in \mathcal{X}_{4 / 3}$.

From (4.4) we know $\nabla c \in L^{\infty}\left(L^{4}\right) \cap L^{20 / 7}\left(L^{60}\right)$. Moreover, from Lemma A.1 with $p_{1}=\infty, q_{1}=4, p_{2}=$ $20 / 7, q_{2}=60$ and $p=3$ we obtain

$$
\nabla c \in L^{\infty}\left(L^{4}\right) \cap L^{3}\left(L^{36}\right) .
$$

Therefore, from (4.8), $\nabla c \cdot \nabla n \in L^{25 / 12}\left(L^{100 / 93}\right) \cap L^{75 / 61}\left(L^{900 / 637}\right)$. Finally, from Lemma A.1 with $\left(p_{1}, q_{1}\right)=(25 / 12,100 / 93),\left(p_{2}, q_{2}\right)=(75 / 61,900 / 637)$ we have $\nabla c \cdot \nabla n \in L^{4 / 3}\left(L^{4 / 3}\right)$. In a similar way, from (4.8) and $u \in \mathcal{X}_{2}$, we have $u \cdot \nabla n \in L^{4 / 3}\left(L^{4 / 3}\right)$. From Theorem 2.1 we conclude that $n \in \mathcal{X}_{4 / 3}$.

x) $\nabla n \in L^{8 / 3}\left(L^{3 / 2}\right)$.

Since $n \in \mathcal{X}_{4 / 3}$, we have $n \in L^{\infty}\left(W^{1 / 2,4 / 3}\right) \cap L^{4 / 3}\left(W^{2,4 / 3}\right)$. Then,

$$
D^{1 / 2} n \in L^{\infty}\left(L^{4 / 3}\right) \cap L^{4 / 3}\left(W^{3 / 2,4 / 3}\right) \hookrightarrow L^{\infty}\left(L^{4 / 3}\right) \cap L^{4 / 3}\left(W^{1,12 / 7}\right) .
$$

Thus, from Lemma A.5 with $\alpha=1 / 2, \beta=1, \gamma=0, p=12 / 7, q=4 / 3$, we have $\lambda=1 / 2, r=3 / 2$ and for $s>0$,

$$
\left\|D^{1 / 2} n\right\|_{W^{1 / 2,3 / 2}}^{s} \leq\left\|D^{1 / 2} n\right\|_{W^{1,12 / 7}}^{s \lambda}\left\|D^{1 / 2} n\right\|_{L^{4 / 3}}^{s(-\lambda)}
$$

Since $D^{1 / 2} n \in L^{4 / 3}$ in time, we have $s \lambda=4 / 3$, which implies $s=8 / 3$ and then $n \in L^{8 / 3}\left(W^{1,3 / 2}\right)$. Finally,

$$
\nabla n \in L^{8 / 3}\left(L^{3 / 2}\right)
$$

xi) $n \in \mathcal{X}_{10 / 7}$.

From (4.4) we know $\nabla c \in L^{\infty}\left(L^{4}\right) \cap L^{20 / 7}\left(L^{60}\right)$. Moreover, from Lemma A.1 with $p_{1}=\infty, q_{1}=4, p_{2}=$ $20 / 7, q_{2}=60$ and $p=3$, again

$$
\nabla c \in L^{\infty}\left(L^{4}\right) \cap L^{3}\left(L^{36}\right) .
$$

Therefore, from (4.9), $\nabla c \cdot \nabla n \in L^{8 / 3}\left(L^{12 / 11}\right) \cap L^{24 / 17}\left(L^{36 / 25}\right)$. Finally, from Lemma A.1 with $\left(p_{1}, q_{1}\right)=$ $(8 / 3,12 / 11),\left(p_{2}, q_{2}\right)=(24 / 17,36 / 25)$ we have $\nabla c \cdot \nabla n \in L^{10 / 7}\left(L^{10 / 7}\right)$. In a similar way, from (4.9) and $u \in \mathcal{X}_{2}$, we have $u \cdot \nabla n \in L^{10 / 7}\left(L^{10 / 7}\right)$. From Theorem 2.1 we conclude that $n \in \mathcal{X}_{10 / 7}$.

xii) $\nabla n \in L^{2}\left(L^{2}\right)$ and $n \in L^{10 / 3}\left(L^{10 / 3}\right)$.

Since $n \in \mathcal{X}_{10 / 7}$, we have $n \in L^{\infty}\left(W^{3 / 5,10 / 7}\right) \cap L^{10 / 7}\left(W^{2,10 / 7}\right)$. Then,

$$
D^{3 / 5} n \in L^{\infty}\left(L^{10 / 7}\right) \cap L^{10 / 7}\left(W^{7 / 5,10 / 7}\right) \hookrightarrow L^{\infty}\left(L^{10 / 7}\right) \cap L^{10 / 7}\left(W^{1,30 / 17}\right) .
$$


Thus, from Lemma A.5 with $\alpha=2 / 5, \beta=1, \gamma=0, p=30 / 17, q=10 / 7$, we have $\lambda=2 / 5, r=150 / 97$ and for $s>0$,

$$
\left\|D^{3 / 5} n\right\|_{W^{2 / 5,150 / 97}}^{s} \leq\left\|D^{3 / 5} n\right\|_{W^{1,30 / 17}}^{s \lambda}\left\|D^{3 / 5} n\right\|_{L^{10 / 7}}^{s(1-\lambda)}
$$

Since $D^{3 / 5} n \in L^{10 / 7}$ in time, we have $s \lambda=10 / 7$, which implies $s=25 / 7$ and then $n \in L^{25 / 7}\left(W^{1,150 / 97}\right)$. Now, by Sobolev embeddings $\nabla n \in L^{10 / 7}\left(W^{1,10 / 7}\right) \hookrightarrow L^{10 / 7}\left(L^{30 / 11}\right)$. That is $\nabla n \in L^{25 / 7}\left(L^{150 / 97}\right) \cap$ $L^{10 / 7}\left(L^{30 / 11}\right)$. Then from Lemma A.1 with $p_{1}=25 / 7, q_{1}=150 / 97, p_{2}=10 / 7, q_{2}=30 / 11$ and $p=2$,

$$
\nabla n \in L^{2}\left(L^{2}\right) .
$$

Finally, by Sobolev embeddings we have $n \in L^{\infty}\left(W^{3 / 5,10 / 7}\right) \cap L^{10 / 7}\left(W^{2,10 / 7}\right) \hookrightarrow L^{\infty}\left(L^{2}\right) \cap L^{10 / 7}\left(L^{30}\right) \hookrightarrow$ $L^{10 / 3}\left(L^{10 / 3}\right)$.

xiii) $n \in \mathcal{X}_{20 / 13}$

Since we had $\nabla c \in L^{20 / 3}\left(L^{20 / 3}\right), \Delta c \in L^{20 / 7}\left(L^{20 / 7}\right)$ and $u \in L^{10}\left(L^{10}\right) \hookrightarrow L^{20 / 3}\left(L^{20 / 3}\right)$, from the regularity of $n$ obtained in last item we have

$$
u \cdot \nabla n, \nabla c \cdot \nabla n, n \Delta c \in L^{20 / 13}\left(L^{20 / 13}\right) .
$$

We conclude, from Theorem 2.1, $n \in \mathcal{X}_{20 / 13}$.

xiv) $n \in L^{\infty}\left(L^{2}\right) \cap L^{2}\left(H^{1}\right)$.

Since $n \in \mathcal{X}_{20 / 13}$, we have $n \in L^{\infty}\left(W^{7 / 10,20 / 13}\right) \cap L^{20 / 13}\left(W^{2,20 / 13}\right)$. Then, from (A.1) in the appendix we have

$$
n \in L^{\infty}\left(W^{7 / 10,20 / 13}\right) \cap L^{20 / 13}\left(W^{2,20 / 13}\right) \hookrightarrow L^{\infty}\left(H^{1 / 4}\right) \cap L^{20 / 13}\left(H^{31 / 20}\right) .
$$

Then, from Lemma A.3 with $\left(p_{1}, s_{1}\right)=(\infty, 1 / 4),\left(p_{2}, s_{2}\right)=(20 / 13,31 / 20)$ we have $n \in L^{2}\left(H^{65 / 52}\right) \hookrightarrow$ $L^{2}\left(H^{1}\right)$. From Step 3-xii), we already had $n \in L^{\infty}\left(L^{2}\right)$.

Now we go back to improve the regularity of $c$.

Step 4: $c \in \mathcal{X}_{10 / 3}$.

i) $c \in \mathcal{X}_{10 / 3}$.

Since $n \in L^{\infty}\left(L^{2}\right) \cap L^{2}\left(H^{1}\right) \hookrightarrow L^{10 / 3}\left(L^{10 / 3}\right)$ and $u \in L^{10}\left(L^{10}\right), g \in L^{4}\left(L^{4}\right), c \in L^{\infty}\left(L^{\infty}\right), \quad \nabla c \in$ $L^{20 / 3}\left(L^{20 / 3}\right)$ imply $u \cdot \nabla c, g c \in L^{4}\left(L^{4}\right), c n \in L^{10 / 3}\left(L^{10 / 3}\right)$, we conclude, from Theorem $2.1, c \in \mathcal{X}_{10 / 3}$.

ii) $\nabla c \in L^{10}\left(L^{10}\right)$ and $\Delta c \in L^{10 / 3}\left(L^{10 / 3}\right)$.

Since $c \in \mathcal{X}_{10 / 3}$, we have $\nabla c \in L^{\infty}\left(W^{2 / 5,10 / 3}\right) \cap L^{10 / 3}\left(W^{1,10 / 3}\right) \hookrightarrow L^{\infty}\left(L^{6}\right) \cap L^{10 / 3}\left(W^{1,10 / 3}\right)$. Thus, from Lemma A.4 with $q_{1}=6, p_{1}=p_{2}=10 / 3, r=1$, we obtain $\nabla c \in L^{10}\left(L^{10}\right)$. The fact that $\Delta c \in L^{10 / 3}\left(L^{10 / 3}\right)$ is immediate from the definition of $\mathcal{X}_{10 / 3}$.

Step 5: $n \in \mathcal{X}_{5 / 3}$.

i) $n \in \mathcal{X}_{5 / 3}$.

From the regularities of $n$ in Step 3-xii) and $c$ in Step 4-ii), and since $c \in L^{\infty}\left(L^{\infty}\right), u \in L^{10}\left(L^{10}\right)$ we obtain $u \cdot \nabla n, n \Delta c, \nabla c \cdot \nabla n \in L^{5 / 3}\left(L^{5 / 3}\right)$. Therefore, from Theorem 2.1, $n \in \mathcal{X}_{5 / 3}$.

ii) $n \in L^{5}\left(L^{5}\right)$ and $\nabla n \in L^{20 / 9}\left(L^{20 / 9}\right)$.

Since $n \in \mathcal{X}_{5 / 3}$, we have $n \in L^{\infty}\left(W^{4 / 5,5 / 3}\right) \cap L^{5 / 3}\left(W^{2,5 / 3}\right) \hookrightarrow L^{\infty}\left(L^{3}\right) \cap L^{5 / 3}\left(W^{2,5 / 3}\right)$. Thus, from Lemma A.4 with $q_{1}=3, p_{1}=p_{2}=5 / 3, r=2$, we obtain $n \in L^{5}\left(L^{5}\right)$. On the other hand, since $n \in L^{\infty}\left(W^{4 / 5,5 / 3}\right) \cap L^{5 / 3}\left(W^{2,5 / 3}\right)$, from (A.1) in the appendix we have

$$
n \in L^{\infty}\left(H^{1 / 2}\right) \cap L^{5 / 3}\left(H^{17 / 10}\right) .
$$


Then, from Lemma A.3 with $\left(p_{1}, s_{1}\right)=(\infty, 1 / 2),\left(p_{2}, s_{2}\right)=(5 / 3,17 / 10)$ we have $n \in L^{20 / 9}\left(H^{7 / 5}\right)$. In particular $\nabla n \in L^{20 / 9}\left(H^{2 / 5}\right) \hookrightarrow L^{20 / 9}\left(L^{20 / 9}\right)$.

Step 6: $c \in \mathcal{X}_{4}$.

i) $c \in \mathcal{X}_{4}$.

We already have from Step 4-i) that $u \cdot \nabla c, g c \in L^{4}\left(L^{4}\right)$. Since $c \in L^{\infty}\left(L^{\infty}\right)$, and from Step 5-ii), $n \in L^{5}\left(L^{5}\right)$, we obtain $c n \in L^{5}\left(L^{5}\right)$. Thus, from Theorem 2.1, $c \in \mathcal{X}_{4}$. Moreover, from (4.3),

$$
\begin{aligned}
\|c\|_{\mathcal{X}_{4}} & \leq C\left(\|-u \cdot \nabla c-c n+g c\|_{L^{4}\left(L^{4}\right)}+\left\|c_{0}\right\|_{\widehat{W}^{3 / 2,4}}\right) \\
& \leq C\left(\|u\|_{L^{10}\left(L^{10}\right)}\|\nabla c\|_{L^{20 / 3}\left(L^{20 / 3}\right)}+\|c\|_{L^{\infty}\left(L^{\infty}\right)}\left(\|n\|_{L^{5}\left(L^{5}\right)}+\|g\|_{L^{4}\left(L^{4}\right)}\right)+\left\|c_{0}\right\|_{\widehat{W}^{3 / 2,4}}\right) \\
& \leq C\left(\left\|n_{0}\right\|_{\widehat{W}^{3 / 2,4}},\left\|c_{0}\right\|_{\widehat{W}^{3 / 2,4}},\left\|u_{0}\right\|_{V},\|g\|_{L^{4}\left(L^{4}\right)},\|f\|_{L^{2}\left(L^{2}\right)},\|n\|_{L^{2}\left(L^{2}\right)},\|u\|_{L^{8}\left(L^{4}\right)}\right) .
\end{aligned}
$$

ii) $\nabla c \in L^{20}\left(L^{20}\right)$ and $\Delta c \in L^{4}\left(L^{4}\right)$.

Since $c \in \mathcal{X}_{4}$, we have $\nabla c \in L^{\infty}\left(W^{1 / 2,4}\right) \cap L^{4}\left(W^{1,4}\right) \hookrightarrow L^{\infty}\left(L^{12}\right) \cap L^{4}\left(W^{1,4}\right)$. Thus, from Lemma A.4 with $q_{1}=12, p_{1}=p_{2}=4, r=1$, we obtain $\nabla c \in L^{20}\left(L^{20}\right)$. The fact that $\Delta c \in L^{4}\left(L^{4}\right)$ is immediate from the definition of $\mathcal{X}_{4}$.

Notice that the regularity of $c$ cannot be improved, because $g \in L^{4}\left(L^{4}\right)$ by hypothesis.

Step 7: $n \in \mathcal{X}_{4}$.

i) $n \in \mathcal{X}_{20 / 11}$.

From the regularities of $n$ in Step 5-ii), and $c$ in Step 6-ii), and since $u \in L^{10}\left(L^{10}\right)$, we obtain $u \cdot \nabla n \in$ $L^{20 / 11}\left(L^{20 / 11}\right), n \Delta c \in L^{20 / 9}\left(L^{20 / 9}\right), \nabla c \cdot \nabla n \in L^{2}\left(L^{2}\right)$. Therefore, from Theorem 2.1, $n \in \mathcal{X}_{20 / 11}$.

ii) $\nabla n \in L^{5 / 2}\left(L^{5 / 2}\right)$.

Since $n \in \mathcal{X}_{20 / 11}$, we have $n \in L^{\infty}\left(W^{9 / 10,20 / 11}\right) \cap L^{20 / 11}\left(W^{2,20 / 11}\right)$. Then, from (A.1) in the appendix we have

$$
n \in L^{\infty}\left(W^{9 / 10,20 / 11}\right) \cap L^{20 / 11}\left(W^{2,20 / 11}\right) \hookrightarrow L^{\infty}\left(H^{4 / 3}\right) \cap L^{20 / 11}\left(H^{20 / 37}\right) .
$$

Then, from Lemma A.3 with $\left(p_{1}, s_{1}\right)=(\infty, 4 / 3),\left(p_{2}, s_{2}\right)=(20 / 11,20 / 37)$ we have $n \in L^{5 / 2}\left(H^{31 / 20}\right)$. In particular $\nabla n \in L^{5 / 2}\left(H^{11 / 20}\right) \hookrightarrow L^{5 / 2}\left(L^{60 / 19}\right) \hookrightarrow L^{5 / 2}\left(L^{5 / 2}\right)$.

iii) $n \in \mathcal{X}_{2}$.

Since $\nabla n \in L^{5 / 2}\left(L^{5 / 2}\right)$ and $u \in L^{10}\left(L^{10}\right)$ we have $u \cdot \nabla n \in L^{2}\left(L^{2}\right)$. Then, following Step 7-i), from Theorem 2.1, $n \in \mathcal{X}_{2}$.

iv) $n \in \mathcal{X}_{5 / 2}$.

We have $n \in \mathcal{X}_{2} \hookrightarrow L^{10}\left(L^{10}\right)$. Moreover $\nabla n \in \mathcal{X}_{1} \hookrightarrow L^{10 / 3}\left(L^{10 / 3}\right)$ where we used Lemma A.4 with $q_{1}=$ $p_{1}=p_{2}=2, r=1$. Therefore, from the regularity of $c$ in Step 6-ii) and $u \in L^{10}\left(L^{10}\right)$ we obtain $u \cdot \nabla n \in$ $L^{5 / 2}\left(L^{5 / 2}\right)$ and $\nabla n \cdot \nabla c, n \Delta c \in L^{20 / 7}\left(L^{20 / 7}\right)$. Since $\frac{5}{2}<\frac{20}{7}$ we get $n \in \mathcal{X}_{5 / 2}$.

v) $\nabla n \in L^{4}\left(L^{4}\right)$.

Since $n \in \mathcal{X}_{5 / 2}$, we have $\nabla n \in L^{\infty}\left(W^{1 / 5,5 / 2}\right) \cap L^{5 / 2}\left(W^{1,5 / 2}\right)$. Then, from (A.1) in the appendix we have

$$
n \in L^{\infty}\left(W^{1 / 5,5 / 2}\right) \cap L^{5 / 2}\left(W^{1,5 / 2}\right) \hookrightarrow L^{\infty}\left(H^{1 / 2}\right) \cap L^{5 / 2}\left(H^{13 / 10}\right) .
$$

Then, from Lemma A.3 with $\left(p_{1}, s_{1}\right)=(\infty, 1 / 2),\left(p_{2}, s_{2}\right)=(5 / 2,13 / 10)$ we have $\nabla n \in L^{4}\left(H^{1}\right) \hookrightarrow L^{4}\left(L^{4}\right)$. vi) $n \in \mathcal{X}_{20 / 7}$.

Since $\nabla n \in L^{4}\left(L^{4}\right)$ and $u \in L^{10}\left(L^{10}\right)$, we have $u \cdot \nabla n \in L^{20 / 7}\left(L^{20 / 7}\right)$. Then, following Step 7 -iv), from Theorem 2.1, $n \in \mathcal{X}_{20 / 7}$.

vii) $n \in L^{\infty}\left(L^{\infty}\right)$ and $\nabla n \in L^{20 / 3}\left(L^{20 / 3}\right)$.

Since $n \in \mathcal{X}_{20 / 7}$, we have $n \in L^{\infty}\left(W^{13 / 10,20 / 7}\right) \hookrightarrow L^{\infty}\left(L^{\infty}\right)$. Moreover, from Lemma A.4 in the appendix 
we have

$$
\nabla n \in L^{\infty}\left(W^{3 / 10,20 / 7}\right) \cap L^{20 / 7}\left(W^{1,20 / 7}\right) \hookrightarrow L^{20 / 3}\left(L^{20 / 3}\right) .
$$

viii) $n \in \mathcal{X}_{4}$.

From the corresponding regularities of $c$ and $n$ in previous steps, and since $u \in L^{10}\left(L^{10}\right)$ we obtain $u \cdot \nabla n \in L^{4}\left(L^{4}\right), n \Delta c \in L^{4}\left(L^{4}\right)$ and $\nabla n \cdot \nabla c \in L^{5}\left(L^{5}\right)$. Thus, by Theorem 2.1, $n \in \mathcal{X}_{4}$. Moreover, from $(4.11)$,

$$
\begin{aligned}
\|n\|_{\mathcal{X}_{4}} & \leq C\left(\|u \cdot \nabla n+\nabla \cdot(n \nabla c)\|_{L^{4}\left(L^{4}\right)}+\left\|n_{0}\right\|_{\widehat{W}^{3 / 2,4}}\right) \\
& \leq C\left(\left\|n_{0}\right\|_{\widehat{W}^{3 / 2,4}},\left\|c_{0}\right\|_{\widehat{W}^{3 / 2,4}},\left\|u_{0}\right\|_{V},\|g\|_{L^{4}\left(L^{4}\right)},\|f\|_{L^{2}\left(L^{2}\right)},\|n\|_{L^{2}\left(L^{2}\right)},\|u\|_{L^{8}\left(L^{4}\right)}\right) .
\end{aligned}
$$

Note that the regularity of $n$ cannot be improved, since $\Delta c \in L^{4}\left(L^{4}\right)$ is the best we can get.

Finally, we observe that estimate (4.2) follows from Theorem 2.1 and the computations above.

Remark 4.3. Notice that, following the proof above, if we assume $g \in L^{10 / 3}\left(L^{10 / 3}\right)$, we can get a strong solution in the class $\mathcal{X}_{5 / 3} \times \mathcal{X}_{10 / 3} \times \mathcal{X}_{2}$.

Before formulating the optimal control problem, let us state the following result concerning a "linearized" version of System (1.2).

Lemma 4.4. Let $\left[\bar{n}_{0}, \bar{c}_{0}, \bar{u}_{0}\right] \in \widehat{W}^{3 / 2,4} \times \widehat{W}^{3 / 2,4} \times V,[w, v] \in L^{4}\left(L^{4}\right) \times L^{2}\left(L^{2}\right)$, and $[n, c, u] \in \mathcal{X}_{4} \times \mathcal{X}_{4} \times \mathcal{X}_{2}$ with $\operatorname{div} u=0$ in $\Omega_{T}$. Then, there exists a unique element $[\bar{n}, \bar{c}, \bar{u}] \in \mathcal{X}_{4} \times \mathcal{X}_{4} \times \mathcal{X}_{2}$ and some $\pi \in L^{2}\left(H^{1}\right)$ solution of the following problem:

$$
\left\{\begin{array}{l}
\bar{n}_{t}+\bar{u} \cdot \nabla n+u \cdot \nabla \bar{n}=\Delta \bar{n}-\chi \nabla \cdot(\bar{n} \nabla c)-\chi \nabla \cdot(n \nabla \bar{c}), \\
\bar{c}_{t}+\bar{u} \cdot \nabla c+u \cdot \nabla \bar{c}=\Delta \bar{c}-\gamma \bar{c} n-\gamma c \bar{n}+w \bar{c} \\
\bar{u}_{t}+(\bar{u} \cdot \nabla) u+(u \cdot \nabla) \bar{u}=\Delta \bar{u}-\nabla \pi-\bar{n} \nabla \Phi+v \\
\nabla \cdot \bar{u}=0
\end{array}\right.
$$

for some $\pi \in L^{2}\left(H^{1}\right)$, which is unique up to the addition of a function of $L^{2}(0, T)$.

Proof. The idea is to use the Leray-Schauder fixed-point theorem. Indeed, let $X=L^{\infty}\left(L^{2}\right) \cap L^{2}\left(H^{1}\right)$ and $\widetilde{\Gamma}$ be the operator

$$
\begin{aligned}
\widetilde{\Gamma}: X \times X & \rightarrow \mathcal{X}_{5 / 3} \times X_{5 / 3} \\
{\left[n^{d}, c^{d}\right] } & \mapsto[\bar{n}, \bar{c}],
\end{aligned}
$$

where $[\bar{n}, \bar{c}]$ is the solution of the coupled system

$$
\left\{\begin{array}{l}
\bar{n}_{t}+\bar{u} \cdot \nabla n+u \cdot \nabla n^{d}=\Delta \bar{n}-\chi \nabla \cdot\left(n^{d} \nabla c\right)-\chi \nabla \cdot(n \nabla \bar{c}), \\
\bar{c}_{t}+\bar{u} \cdot \nabla c+u \cdot \nabla c^{d}=\Delta \bar{c}-\gamma c^{d} n-\gamma c n^{d}+w c^{d} \\
\bar{u}_{t}+(\bar{u} \cdot \nabla) u+(u \cdot \nabla) \bar{u}=\Delta \bar{u}-\nabla \pi+n^{d} \nabla \Phi+v \\
\nabla \cdot \bar{u}=0
\end{array}\right.
$$

Following [6], Theorem 4.2.1, since $v \in L^{2}\left(L^{2}\right)$ and $n^{d} \in X \hookrightarrow L^{10 / 3}\left(L^{10 / 3}\right)$, there exists a unique solution $\bar{u} \in \mathcal{X}_{2} \hookrightarrow L^{10}\left(L^{10}\right)$ of $(4.14)_{3}$. This implies $\bar{u} \cdot \nabla c \in L^{20 / 3}\left(L^{20 / 3}\right)$ and we can follow the ideas in the proof of Proposition 3.5 to prove the existence of a unique solution $[\bar{n}, \bar{c}] \in X \times X$.

The extra regularity of system (4.13) can be obtained by a bootstrapping argument as follows: 
Step 1: $\bar{c} \in \mathcal{X}_{20 / 9}$. Following Step 5-ii) in the proof of Theorem 4.2, $\bar{n}, \bar{c} \in L^{5}\left(L^{5}\right)$ and $\nabla \bar{n}, \nabla \bar{c} \in L^{20 / 9}\left(L^{20 / 9}\right)$. Together with the regularities $n, c, u \in \mathcal{X}_{4} \hookrightarrow L^{\infty}\left(L^{\infty}\right), \nabla c \in L^{20}\left(L^{20}\right)$, and $w \in L^{4}\left(L^{4}\right)$ give $c \bar{n}, \bar{c} n \in L^{5}\left(L^{5}\right)$, $w c \in L^{4}\left(L^{4}\right), \bar{u} \cdot \nabla c \in L^{20 / 3}\left(L^{20 / 3}\right)$ and $u \cdot \nabla \bar{c} \in L^{20 / 9}\left(L^{20 / 9}\right)$. Therefore, from Theorem 2.1, $\bar{c} \in \mathcal{X}_{20 / 9}$.

Step 2: $\bar{n} \in \mathcal{X}_{20 / 11}$. By definition $\Delta \bar{c} \in L^{20 / 9}\left(L^{20 / 9}\right)$. Moreover, from Step 2-ii) in the proof of Theorem 4.2, $\bar{c} \in L^{20}\left(L^{20}\right), \nabla \bar{c} \in L^{4}\left(L^{4}\right)$. Together with the regularities above give $\nabla n \cdot \nabla \bar{c} \in L^{10 / 3}\left(L^{10 / 3}\right), \nabla c \cdot \nabla \bar{n} \in L^{2}\left(L^{2}\right)$, $\bar{n} \Delta c, n \Delta \bar{c} \in L^{20 / 9}\left(L^{20 / 9}\right), \bar{u} \cdot \nabla n \in L^{20 / 3}\left(L^{20 / 3}\right)$ and $u \cdot \nabla \bar{n} \in L^{20 / 11}\left(L^{20 / 11}\right)$. Therefore, from Theorem 2.1, $\bar{n} \in \mathcal{X}_{20 / 11}$.

Step 3: $\bar{n} \in \mathcal{X}_{2}$. From Step 7-ii) in the proof of Theorem 4.2, $\nabla \bar{n} \in L^{5 / 2}\left(L^{5 / 2}\right)$. Therefore, $u \cdot \nabla \bar{n} \in L^{2}\left(L^{2}\right)$. Together with the regularities given in Step 2, from Theorem 2.1, $\bar{n} \in \mathcal{X}_{2}$.

Step 4: $\bar{c} \in \mathcal{X}_{4}$. Following Step 2-ii) in the proof of Theorem 4.2, $\bar{c} \in L^{20}\left(L^{20}\right)$ and $\nabla \bar{c} \in L^{4}\left(L^{4}\right)$. Then $u \cdot \nabla \bar{c} \in L^{4}\left(L^{4}\right)$. Therefore, from the regularities in Step 1, and Theorem 2.1, $\bar{c} \in \mathcal{X}_{4}$.

Step 5: $\bar{n} \in \mathcal{X}_{4}$. The proof follows Step 7 in the proof of Theorem 4.2.

Finally, for the uniqueness part, let $\left[\bar{n}_{1}, \bar{c}_{1}, \bar{u}_{1}, \pi_{1}\right],\left[\bar{n}_{2}, \bar{c}_{2}, \bar{u}_{2}, \pi_{2}\right] \in \mathcal{X}_{4} \times \mathcal{X}_{4} \times \mathcal{X}_{2} \times L^{2}\left(H^{1}\right)$ be two solutions of (4.13). Then, defining $\widetilde{u}=\bar{u}_{1}-\bar{u}_{2}, \widetilde{n}=\bar{n}_{1}-\bar{n}_{2}, \widetilde{c}=\bar{c}_{1}-\bar{c}_{2}, \widetilde{u}=\bar{u}_{1}-\bar{u}_{2}$ and $\tilde{\pi}=\pi_{1}-\pi_{2}$, we have

$$
\left\{\begin{array}{l}
\widetilde{n}_{t}-\Delta \widetilde{n}+\widetilde{u} \cdot \nabla n=-u \cdot \nabla \widetilde{n}-\chi \nabla \cdot(\widetilde{n} \nabla c+n \nabla \widetilde{c}), \\
\widetilde{c}_{t}-\Delta \widetilde{c}+\widetilde{u} \cdot \nabla c=-u \cdot \nabla \widetilde{c}-\gamma \widetilde{c} n-\gamma c \widetilde{n}+w \widetilde{c}, \\
\widetilde{u}_{t}-\Delta \widetilde{u}+(\widetilde{u} \cdot \nabla) u+(u \cdot \nabla) \widetilde{u}+\nabla \tilde{\pi}=\widetilde{n} \nabla \Phi, \\
\widetilde{n}(0)=\widetilde{c}(0)=\widetilde{u}(0)=0
\end{array}\right.
$$

with the corresponding boundary conditions. Proceeding as we did in the proof of Step $\mathbf{5}$ - Proposition 3.5, is not difficult to see that

$$
\begin{gathered}
\frac{1}{2} \frac{\mathrm{d}}{\mathrm{d} t}\|\widetilde{n}\|_{L^{2}}^{2}+\|\nabla \widetilde{n}\|_{L^{2}}^{2} \leq \delta\|\nabla \widetilde{n}\|_{L^{2}}^{2}+C_{\delta}\|n\|_{L^{\infty}}^{2}\left(\|\widetilde{u}\|_{L^{2}}^{2}+\|\nabla \widetilde{c}\|_{L^{2}}^{2}\right)+C_{\delta}\left(\|\nabla c\|_{L^{4}}^{8}+\|\nabla c\|_{L^{4}}^{2}\right)\|\widetilde{n}\|_{L^{2}}^{2}, \\
\frac{1}{2} \frac{\mathrm{d}}{\mathrm{d} t}\|\widetilde{u}\|_{L^{2}}^{2}+\|\nabla \widetilde{u}\|_{L^{2}}^{2} \leq \delta\|\nabla \widetilde{u}\|_{L^{2}}^{2}+C_{\delta}\|\nabla u\|_{L^{2}}^{4}\|\widetilde{u}\|_{L^{2}}^{2}+\frac{1}{2}\|\nabla \Phi\|_{L^{\infty}}^{2}\|\widetilde{n}\|_{L^{2}}^{2}+\frac{1}{2}\|\widetilde{u}\|_{L^{2}}^{2}, \\
\frac{1}{2} \frac{\mathrm{d}}{\mathrm{d} t}\|\widetilde{c}\|_{L^{2}}^{2}+\|\nabla \widetilde{c}\|_{L^{2}}^{2} \leq \delta\|\nabla \widetilde{c}\|_{L^{2}}^{2}+C_{\delta}\left(\|c\|_{L^{\infty}}^{2}\|\widetilde{u}\|_{L^{2}}^{2}+\left(\|w\|_{L^{4}}^{8 / 5}+\|w\|_{L^{4}}\right)\|\widetilde{c}\|_{L^{2}}^{2}\right) \\
+\gamma\|n\|_{L^{\infty}}\|\widetilde{c}\|_{L^{2}}^{2}+\frac{\gamma}{2}\|c\|_{L^{\infty}}\left(\|\widetilde{n}\|_{L^{2}}^{2}+\|\widetilde{c}\|_{L^{2}}^{2}\right),
\end{gathered}
$$

for some $\delta>0$. Putting together the inequalities above and using the Gronwall inequality, we can obtain $\widetilde{n}=\widetilde{c}=\widetilde{u}=0$ a.e $(x, t) \in \Omega \times(0, T)$.

As a consequence of the last lemma, and following the ideas in [5], the next corollary states that the set of controls, around a given control providing a strong solution, is an open set.

Corollary 4.5. If system (1.2) has a solution $[n, c, u] \in \mathcal{X}_{4} \times \mathcal{X}_{4} \times \mathcal{X}_{2}$ for some element $(\bar{g}, \bar{f}) \in L^{4}\left(L^{4}\right) \times L^{2}\left(L^{2}\right)$, then there exists an open neighborhood $\mathcal{A}_{0} \times \mathcal{B}_{0}$ of $[\bar{g}, \bar{f}]$ in $L^{4}\left(L^{4}\right) \times L^{2}\left(L^{2}\right)$ such that $(1.2)$ has a strong solution for every $(g, f) \in \mathcal{A}_{0} \times \mathcal{B}_{0}$. Moreover, the mapping $G: \mathcal{A}_{0} \times \mathcal{B}_{0} \rightarrow \mathcal{X}_{4} \times \mathcal{X}_{4} \times \mathcal{X}_{2}$, defined by $G([g, f])=[n, c, u]$, is of class $C^{\infty}$. Finally, if $[\bar{n}, \bar{c}, \bar{u}]=G^{\prime}([g, f])[w, v]$, for some $[g, f] \in \mathcal{A}_{0} \times \mathcal{B}_{0}$ and some $[w, v] \in L^{4}\left(L^{4}\right) \times L^{2}\left(L^{2}\right)$, then $[\bar{n}, \bar{c}, \bar{u}]$ is the unique strong solution of the problem:

$$
\left\{\begin{array}{l}
\bar{n}_{t}+\bar{u} \cdot \nabla n+u \cdot \nabla \bar{n}=\Delta \bar{n}-\chi \nabla \cdot(\bar{n} \nabla c)-\chi \nabla \cdot(n \nabla \bar{c}), \\
\bar{c}_{t}+\bar{u} \cdot \nabla c+u \cdot \nabla \bar{c}=\Delta \bar{c}-\gamma \bar{c} n-\gamma c \bar{n}+w \bar{c} \\
\bar{u}_{t}+(\bar{u} \cdot \nabla) u+(u \cdot \nabla) \bar{u}=\Delta \bar{u}-\nabla \pi+\bar{n} \nabla \Phi+v \\
\nabla \cdot \bar{u}=0
\end{array}\right.
$$


with boundary conditions for $\frac{\partial \bar{n}}{\partial \nu}=\frac{\partial \bar{c}}{\partial \nu}=0, \bar{u}=0$ for some $\pi \in L^{2}\left(H^{1}\right)$, which is unique up to the addition of a function of $L^{2}(0, T)$.

Proof. We outline the main steps of the proof. Let us consider the space

$$
\mathcal{H}=\left\{\mathcal{X}_{2}: u_{t} \in L^{2}\left(L_{\sigma}^{2}\right)\right\},
$$

which is a Hilbert space with the norm of $\mathcal{X}_{2}$. We consider the mapping

$$
\mathcal{G}: \mathcal{X}_{4} \times \mathcal{X}_{4} \times \mathcal{H} \times L^{4}\left(L^{4}\right) \times L^{2}\left(L^{2}\right) \longrightarrow L^{4}\left(L^{4}\right) \times L^{4}\left(L^{4}\right) \times L^{2}\left(L^{2}\right) \times \widehat{W}^{3 / 2,4} \times \widehat{W}^{3 / 2,4} \times V,
$$

given by

$$
\begin{aligned}
\mathcal{G}([n, c, u, g, f])= & {\left[n_{t}+u \cdot \nabla n-\Delta n+\chi \nabla \cdot(n \nabla c), c_{t}+u \cdot \nabla c-\Delta c+\gamma c n-g c,\right.} \\
& \left.u_{t}+P[(u \cdot \nabla) u-\Delta u-n \nabla \Phi-f], n(0)-n_{0}, c(0)-c_{0}, u(0)-u_{0}\right],
\end{aligned}
$$

where $P$ is the projection operator.

Then $\mathcal{G}$ is of class $C^{\infty}$ and

$$
\begin{array}{r}
\frac{\partial \mathcal{G}([n, c, u, g, f])}{\partial[n, c, u]}[\bar{n}, \bar{c}, \bar{u}]=\left[\bar{n}_{t}+\bar{u} \cdot \nabla n+u \cdot \nabla \bar{n}-\Delta \bar{n}+\chi \nabla \cdot(\bar{n} \nabla c)+\chi \nabla \cdot(n \nabla \bar{c}),\right. \\
\bar{c}_{t}+\bar{u} \cdot \nabla c+u \cdot \nabla \bar{c}-\Delta \bar{c}+\gamma \bar{c} n+\gamma c \bar{n}-w \bar{c}, \bar{u}_{t}+P[(\bar{u} \cdot \nabla) u+(u \cdot \nabla) \bar{u}-\Delta \bar{u}-\bar{n} \nabla \Phi], \\
\bar{n}(0), \bar{c}(0), \bar{u}(0)] .
\end{array}
$$

Thus, following the ideas in [6], we conclude that (4.15) is satisfied for some $\pi \in L^{2}\left(H^{1}\right)$. In this sense, from Lemma 4.4,

$$
\frac{\partial \mathcal{G}}{\partial[n, c, u]}[n, c, u, g, f]: \mathcal{X}_{4} \times \mathcal{X}_{4} \times \mathcal{H} \longrightarrow L^{4}\left(L^{4}\right) \times L^{4}\left(L^{4}\right) \times L^{2}\left(L^{2}\right) \times \widehat{W}^{3 / 2,4} \times \widehat{W}^{3 / 2,4} \times V
$$

is an isomorphism for every $[n, c, u, g, f] \in \mathcal{X}_{4} \times \mathcal{X}_{4} \times \mathcal{H} \times L^{4}\left(L^{4}\right) \times L^{2}\left(L^{2}\right)$. Then, if problem (1.2) has a strong solution $[n, c, u]$ for a given control $[\bar{g}, \bar{f}]$ then $\mathcal{G}[n, c, u, \bar{g}, \bar{f}]=0$. By applying the implicit function theorem we deduce the existence of an open neighborhood $\mathcal{A}_{0} \times \mathcal{B}_{0}$ of $[\bar{g}, \bar{f}]$ and a mapping $G: \mathcal{A}_{0} \times \mathcal{B}_{0} \rightarrow \mathcal{X}_{4} \times \mathcal{X}_{4} \times \mathcal{H}$ of class $C^{\infty}$ such that $\mathcal{G}([G(g, f), g, f])=0$ for every $[g, f] \in \mathcal{A}_{0} \times \mathcal{B}_{0}$.

As a consequence, the set of controls $[g, f] \in L^{4}\left(L^{4}\right) \times L^{2}\left(L^{2}\right)$ for which there exists a strong solution $[n, c, u]$ is open. This set will be denoted by $\mathcal{A} \times \mathcal{B}$.

\section{The CONTROL PROBLEM}

In this section, we define the bilinear optimal control problem. We derive the optimality system and prove the existence of one global optimal solution. As is explained in [5, 6, 25], allowing the weak solutions of (1.2) to be admissible states imply the optimality system can not be obtained. Indeed, the lack of uniqueness of solutions for the state system imply, eventually, that the Gateaux derivative of the functional is not uniquely determined. That is why the control problem is formulated in such a way that any optimal state is a strong solution of (1.2).

For the rest of this work we fix a finite time $T>0$. We recall the following notation $L^{p}(Y):=L^{p}(0, T ; Y)$. Let

$$
\mathcal{F} \subset L^{4}\left(L^{4}\right) \times L^{2}\left(L^{2}\right), \quad \text { be a nonempty, closed and convex set. }
$$


Let $\left[n_{0}, c_{0}, u_{0}\right] \in \widehat{W}^{3 / 2,4} \times \widehat{W}^{3 / 2,4} \times V$ be an initial data with $n_{0}, c_{0} \geq 0$, and the couple $[g, f] \in \mathcal{F}$ describing the control acting on the $u$-equation and $c$-equation respectively. We define the set of admissible controls as: $\mathcal{U}_{a d}=(\mathcal{A} \times \mathcal{B}) \cap \mathcal{F}$. We consider the functional $J: \mathcal{A} \times \mathcal{B} \rightarrow \mathbb{R}$ defined by

$$
\begin{aligned}
J([g, f])= & \frac{7}{20} \int_{0}^{T}\left\|n(t)-n^{d}(t)\right\|_{L^{20 / 7}}^{20 / 7} \mathrm{~d} t+\frac{1}{2} \int_{0}^{T}\left\|c(t)-c^{d}(t)\right\|_{L^{2}}^{2} \mathrm{~d} t+\frac{1}{8} \int_{0}^{T}\left\|u(t)-u^{d}(t)\right\|_{L^{4}}^{8} \mathrm{~d} t \\
& +\frac{\alpha_{1}}{2} \int_{0}^{T}\|f\|_{L^{2}}^{2} \mathrm{~d} t+\frac{\alpha_{2}}{4} \int_{0}^{T}\|g\|_{L^{4}}^{4} \mathrm{~d} t
\end{aligned}
$$

where $[n, c, u]=G([g, f])$ is the state associated to $[g, f]$, the desired state $\left[n^{d}, c^{d}, u^{d}\right] \in L^{26 / 7}\left(L^{26 / 7}\right) \times L^{4}\left(L^{4}\right) \times$ $L^{14}\left(L^{6}\right)$, and $\alpha_{1}, \alpha_{2}>0$. Then, the control problem is rewritten as

$$
\left\{\begin{array}{l}
\min J([g, f]), \\
{[g, f] \in \mathcal{U}_{a d} .}
\end{array}\right.
$$

The choose of the $L^{20 / 7}\left(L^{20 / 7}\right)$ and $L^{8}\left(L^{4}\right)$ norms for $n$ and $u$, respectively, in the cost functional, is made in line with Theorem 4.2. That is, if $[n, c, u]$ is a weak solution of $(1.2)$ in $(0, T)$ such that $J([g, f])<\infty$, then $[n, u] \in L^{20 / 7}\left(L^{20 / 7}\right) \times L^{8}\left(L^{4}\right)$ and, by Theorem (4.2), $[n, c, u] \in \mathcal{X}_{4} \times \mathcal{X}_{4} \times \mathcal{X}_{2}$ is a strong solution of (1.2) in $(0, T)$. In what follows, we will make the following assumption:

$$
\mathcal{U}_{a d} \neq \emptyset
$$

Remark 5.1. The hypothesis (5.2) holds. Indeed, following [42], given $\epsilon>0$, there exist $\bar{f} \in L^{2}\left(L^{2}\right)$ with $\|\bar{f}\|_{L^{1}\left(L^{1}\right)}<\epsilon$, and a unique solution $u \in \mathcal{X}_{2}$ of

$$
\left\{\begin{array}{l}
u_{t}+(u \cdot \nabla) u=\Delta u-\nabla \pi+\bar{f} \quad \text { in } \Omega \times(0, T), \\
\nabla \cdot u=0, \text { in } \Omega \times(0, T), \\
u=0, \text { on } \partial \Omega \times(0, T), \\
u=u_{0} \text { in } \Omega .
\end{array}\right.
$$

Then, taking $u \in \mathcal{X}_{2}$ the solution of (5.3), we solve the equation $c_{t}-\Delta c=-u \cdot \nabla c$, with Neumann boundary condition $\frac{\partial c}{\partial \nu}=0$, and initial data $c(0)=c_{0}$. In fact, it is clear the existence of a unique weak solution $c \in$ $L^{\infty}\left(L^{2}\right) \cap L^{2}\left(H^{1}\right)$ of this initial and boundary value problem. Then, noting that $u \in \mathcal{X}_{2} \subset L^{10}\left(L^{10}\right)$ and $\nabla c \in$ $L^{2}\left(L^{2}\right)$, it holds that $u \nabla c \in L^{5 / 3}\left(L^{5 / 3}\right)$, which, by parabolic regularity implies that $c \in \mathcal{X}_{5 / 3}$. Even more, proceeding as we did in Lemma 4.4, $c \in \mathcal{X}_{4}$. Next, knowing $u \in \mathcal{X}_{2}$ and $c \in \mathcal{X}_{4}$, we consider the the initial value problem

$$
n_{t}-\Delta n=-u \cdot \nabla n+\chi \nabla \cdot(n \nabla c)
$$

with Neumann boundary condition $\frac{\partial n}{\partial \nu}=0$ and initial data $n(0)=n_{0}$. It is clear that there exists a unique weak solution $n \in L^{\infty}\left(L^{2}\right) \cap L^{2}\left(H^{1}\right) \subset L^{20 / 7}\left(L^{20 / 7}\right)$ for (5.4) with the corresponding initial and Neumann boundary data. Indeed, testing (5.4) by $n$ and integrating by parts, we get

$$
\begin{aligned}
& \frac{1}{2} \frac{\mathrm{d}}{\mathrm{d} t}\|n\|_{L^{2}}^{2}+\|\nabla n\|_{L^{2}}^{2}=-(n \nabla c, \nabla n) \leq C\|\nabla n\|_{L^{2}}\|\nabla c\|_{L^{4}}\|n\|_{L^{4}} \\
& \quad \leq C\|\nabla n\|_{L^{2}}^{7 / 4}\|\nabla c\|_{L^{4}}\|n\|_{L^{2}}^{1 / 2} \leq \delta\|\nabla c\|^{2}+C_{\delta}\|\nabla c\|_{L^{4}}^{8}\|n\|_{L^{2}}^{2} .
\end{aligned}
$$


Thus, we can define $g$ such that $-c n+g c=0$ (i.e $g=n$ ) and $f=\bar{f}+n \nabla \Phi$, which furnish a control pair $[\bar{f}, g] \in \mathcal{U}_{a d}$.

By the regularity of $\left[n^{d}, c^{d}, u^{d}\right]$, and as a consequence of Corollary 4.5, we state now the following differentiability property for the functional $J$.

Theorem 5.2. The functional $J: \mathcal{A} \times \mathcal{B} \rightarrow \mathbb{R}$ is of class $C^{\infty}$, and for every $[g, f] \in \mathcal{A} \times \mathcal{B}$ and $[w, v] \in$ $L^{4}\left(L^{4}\right) \times L^{2}\left(L^{2}\right)$ we have

$$
\left[J^{\prime}(g) w, J^{\prime}(f) v\right]=\left[\int_{0}^{T} \int_{\Omega}\left(\alpha_{2}|g|^{2} g+c q\right) w, \int_{0}^{T} \int_{\Omega}\left(\alpha_{1} f+r\right) v\right]
$$

where $[p, q, r] \in \mathcal{X}_{4} \times \mathcal{X}_{4} \times \mathcal{X}_{2}$ is the unique element satisfying

$$
\left\{\begin{array}{l}
p_{t}+(u \cdot \nabla) p=-\Delta p-\chi \nabla c \cdot \nabla p+\gamma c q+(\nabla \Phi)^{T} r+\left|n-n^{d}\right|^{6 / 7}\left(n-n^{d}\right) \\
q_{t}+(u \cdot \nabla) q=-\Delta q+\chi \nabla \cdot(n \nabla p)+\gamma n q-g q-c+c^{d} \\
r_{t}+(u \cdot \nabla) r-(\nabla u)^{T} r=-\Delta r+\nabla \Pi+(\nabla n)^{T} p+(\nabla c)^{T} q-\left\|u-u^{d}\right\|_{L^{4}}^{4}\left|u-u^{d}\right|^{2}\left(u-u^{d}\right) \\
\nabla \cdot r=0
\end{array}\right.
$$

with $\Pi \in L^{2}\left(0, T ; H^{1}(\Omega)\right)$ uniquely defined up to the addition of a function of $L^{2}(0, T)$.

Note that the assumption on $\left[n^{d}, c^{d}, u^{d}\right]$ implies the right hand side of system (5.5) is in $L^{4}\left(L^{4}\right), L^{4}\left(L^{4}\right)$ and $L^{2}\left(L^{2}\right)$ respectively.

Proof. Let $[g, f] \in L^{4}\left(L^{4}\right) \times L^{2}\left(L^{2}\right)$ such that there exists a strong solution $[n, c, u]$ of $(1.2)$. Let $[\delta g, \delta f] \in$ $L^{4}\left(L^{4}\right) \times L^{2}\left(L^{2}\right)$ be any possible variation of $[g, f]$. Then for $\epsilon>0$ sufficiently small, the solution $\left[n^{\epsilon}, c^{\epsilon}, u^{\epsilon}\right]$, corresponding to

$$
\left[g^{\epsilon}, f^{\epsilon}\right]=[g, f]+\epsilon[\delta g, \delta f]
$$

is such that $\left[n^{\epsilon}, c^{\epsilon}, u^{\epsilon}\right] \in \mathcal{X}_{4} \times \mathcal{X}_{4} \times \mathcal{X}_{2}$ can be written as

$$
\left[n^{\epsilon}, c^{\epsilon}, u^{\epsilon}\right]=[n, c, u]+\epsilon[\delta n, \delta c, \delta u]
$$

where $[\delta n, \delta c, \delta u]$ is the solution of the linearized system:

$$
\left\{\begin{array}{l}
\delta n_{t}+\delta u \cdot \nabla n+u \cdot \nabla \delta n=\Delta \delta n-\chi \nabla \cdot(\delta n \nabla c)-\chi \nabla \cdot(n \nabla \delta c) \\
\delta c_{t}+\delta u \cdot \nabla c+u \cdot \nabla \delta c=\Delta \delta c-\gamma \delta n c-\gamma n \delta c+g \delta c+\delta g c \\
u_{t}+(\delta u \cdot \nabla) u+u \cdot \nabla \delta u=\Delta \delta u+\delta n \nabla \Phi+\delta f \\
\nabla \cdot \delta u=0
\end{array}\right.
$$

In order to derive the dual system, let us multiply last system, respectively, by test functions $[p, q, r]$ such that $\lim _{|x| \rightarrow \infty}[p, q, r]=0$. After integrating in space and time, recalling that $(\cdot, \cdot)$ denotes the product in $L^{2}$ we get

$$
\left\{\begin{array}{l}
\int_{0}^{T}\left[\left(\delta n_{t}, p\right)+\left(\delta u,(\nabla n)^{T} p\right)-(\delta n,(u \cdot \nabla) p)\right] \mathrm{d} t=\int_{0}^{T}[(\delta n, \Delta p)+(\delta n, \chi \nabla c \cdot \nabla p)-(\delta c, \chi \nabla \cdot(n \nabla p))] \mathrm{d} t \\
\int_{0}^{T}\left[\left(\delta c_{t}, q\right)+\left(\delta u,(\nabla c)^{T} q\right)-(\delta c,(u \cdot \nabla) q)\right] \mathrm{d} t=\int_{0}^{T}[(\delta c, \Delta q)-(\delta n, \gamma c q)+(\delta c,-\gamma n q+g q)+(\delta g, c q)] \mathrm{d} t \\
\int_{0}^{T}\left[\left(\delta u_{t}, r\right)+\left(\delta u,(\nabla u)^{T} r\right)-(\delta u,(u \cdot \nabla) r)\right] \mathrm{d} t=\int_{0}^{T}\left[(\delta u, \Delta r)+\left(\delta n,(\nabla \Phi)^{T} r\right)+(\delta f, r)\right] \mathrm{d} t .
\end{array}\right.
$$


By integrating in time and assuming $\left.[p, q, r]\right|_{t=T}=0$ and $\nabla \cdot r=0$ we get

$$
\left\{\begin{array}{l}
\int_{0}^{T}\left[\left(\delta n,-p_{t}-\Delta p-(u \cdot \nabla) p-\chi \nabla c \cdot \nabla p\right)+(\delta c, \chi \nabla \cdot(n \nabla p))+\left(\delta u,(\nabla n)^{T} p\right)\right] \mathrm{d} t=0 \\
\int_{0}^{T}\left[\left(\delta c,-q_{t}-\Delta q-(u \cdot \nabla) q+\gamma n q-g q\right)+(\delta n, \gamma c q)+\left(\delta u,(\nabla c)^{T} q\right)\right] \mathrm{d} t=\int_{0}^{T}[(\delta g, c q)] \mathrm{d} t \\
\int_{0}^{T}\left[\left(\delta u,-r_{t}-\Delta r-(u \cdot \nabla) r+(\nabla u)^{T} r\right)-\left(\delta n,(\nabla \Phi)^{T} r\right)\right] \mathrm{d} t=\int_{0}^{T}[(\delta f, r)] \mathrm{d} t
\end{array}\right.
$$

which, after addition of the three equations become

$$
\begin{aligned}
& \int_{0}^{T}\left[\left(\delta n,-p_{t}-\Delta p-(u \cdot \nabla) p-\chi \nabla c \cdot \nabla p+\gamma c q-(\nabla \Phi)^{T} r\right)\right] \mathrm{d} t \\
& \quad+\int_{0}^{T}\left[\left(\delta c,-q_{t}-\Delta q-(u \cdot \nabla) q+\gamma n q-g q+\chi \nabla \cdot(n \nabla p)\right)\right] \mathrm{d} t \\
& \quad+\int_{0}^{T}\left[\left(\delta u,-r_{t}-\Delta r-(u \cdot \nabla) r+(\nabla u)^{T} r+(\nabla n)^{T} p+(\nabla c)^{T} q\right)\right] \mathrm{d} t \\
& \quad=\int_{0}^{T}[(\delta g, c q)+(\delta f, r)] \mathrm{d} t .
\end{aligned}
$$

Now, the Gateaux derivative of $\frac{7}{20} \int_{0}^{T}\left\|n(t)-n^{d}(t)\right\|_{L^{20 / 7}}^{20 / 7} \mathrm{~d} t$ and $\frac{1}{2} \int_{0}^{T}\left\|c(t)-c^{d}(t)\right\|_{L^{2}}^{2}$ at $[f, g]$, in the direction $\delta n$ and $\delta c$, respectively, is given by

$$
\int_{0}^{T}\left(\left|n-n^{d}\right|^{6 / 7}\left(n-n^{d}\right), \delta n\right) \mathrm{d} t, \quad \int_{0}^{T}\left(c-c^{d}, \delta c\right) \mathrm{d} t .
$$

Likewise, the Gateaux derivative of $\frac{1}{8} \int_{0}^{T}\left\|u(t)-u^{d}(t)\right\|_{L^{4}}^{8}=\frac{1}{8} \int_{0}^{T}\left(\int_{\Omega}\left|u-u^{d}\right|^{4}\right)^{2}$ at $[f, g]$, in the direction $\delta u$ is given by

$$
\int_{0}^{T}\left(\left\|u-u^{d}\right\|_{L^{4}}^{4}\left|u-u^{d}\right|^{2}\left(u-u^{d}\right), \delta u\right) \mathrm{d} t .
$$

Finally, by derivating $\frac{\alpha_{1}}{2} \int_{0}^{T}\|f\|_{L^{2}}^{2} \mathrm{~d} t$ and $\frac{\alpha_{2}}{4} \int_{0}^{T}\|g\|_{L^{4}}^{4} \mathrm{~d} t$, we obtain

$$
\alpha_{1} \int_{0}^{T}(f, \delta f) \mathrm{d} t, \quad \alpha_{2} \int_{0}^{T}\left(|g|^{2} g, \delta g\right) \mathrm{d} t .
$$

From (5.1) and using (5.7)-(5.9), the Gateaux derivative of $J$ at $[f, g]$, in the direction $[\delta f, \delta g]$, is given by

$$
\begin{aligned}
(\delta J \cdot \delta f, \delta J \cdot \delta g)= & \int_{0}^{T}\left(\left|n-n^{d}\right|^{6 / 7}\left(n-n^{d}\right), \delta n\right) \mathrm{d} t+\int_{0}^{T}\left(c-c^{d}, \delta c\right) \mathrm{d} t \\
& +\int_{0}^{T}\left(\left\|u-u^{d}\right\|_{L^{4}}^{4}\left|u-u^{d}\right|^{2}\left(u-u^{d}\right), \delta u\right) \mathrm{d} t+\alpha_{1} \int_{0}^{T}(f, \delta f) \mathrm{d} t+\alpha_{2} \int_{0}^{T}\left(|g|^{2} g, \delta g\right) \mathrm{d} t .(5.1
\end{aligned}
$$

By (5.6) and (5.10), if we consider system (5.5), we obtain the desired result.

Observe that the regularity assumed on the objective $\left[n^{d}, c^{d}, u^{d}\right]$ and the regularity $[n, c, u] \in \mathcal{X}_{4} \times \mathcal{X}_{4} \times \mathcal{X}_{2}$ imply

$$
\left|n-n^{d}\right|^{6 / 7}\left(n-n^{d}\right),\left\|u-u^{d}\right\|_{L^{4}}^{4}\left|u-u^{d}\right|^{2}\left(u-u^{d}\right) \in L^{2}\left(L^{2}\right)
$$


The next theorem establishes the existence of at least one solution of the optimal control problem (P), as well as the first order optimality conditions satisfied by any local minimum of the optimization problem.

Theorem 5.3. If $\mathcal{U}_{a d} \neq \emptyset$, the optimization problem $(\mathrm{P})$ has at least one global optimal solution. Moreover, for any local solution $[\bar{f}, \bar{g}]$, there exist $[\bar{n}, \bar{c}, \bar{u}]$ and $[\bar{p}, \bar{q}, \bar{r}]$ in $\mathcal{X}_{4} \times \mathcal{X}_{4} \times \mathcal{X}_{2}$ and $\widetilde{\pi}, \widetilde{\Pi} \in L^{2}\left(H^{1}\right)$ such that

$$
\begin{gathered}
\left\{\begin{array}{l}
\bar{n}_{t}+\bar{u} \cdot \nabla \bar{n}=\Delta \bar{n}-\chi \nabla \cdot(\bar{n} \nabla \bar{c}), \\
\bar{c}_{t}+\bar{u} \cdot \nabla \bar{c}=\Delta \bar{c}-\gamma \bar{c} \bar{n}+\bar{g} \bar{c} \\
\bar{u}_{t}+(\bar{u} \cdot \nabla) \bar{u}=\Delta \bar{u}-\nabla \bar{\pi}+\bar{n} \nabla \Phi+\bar{f}, \\
\nabla \cdot \bar{u}=0,
\end{array}\right. \\
\left\{\begin{array}{l}
\bar{p}_{t}+(\bar{u} \cdot \nabla) \bar{p}=-\Delta \bar{p}-\chi \nabla \bar{c} \cdot \nabla \bar{p}+\gamma \bar{c} \bar{q}+(\nabla \Phi)^{T} \bar{r}-\left|\bar{n}-n^{d}\right|^{3}\left(\bar{n}-n^{d}\right), \\
\bar{q}_{t}+(\bar{u} \cdot \nabla) \bar{q}=-\Delta \bar{q}+\chi \nabla \cdot(\bar{n} \nabla \bar{p})+\gamma \bar{n} \bar{q}-\bar{g} \bar{q}-\bar{c}+c^{d}, \\
\bar{r}_{t}+(\bar{u} \cdot \nabla) \bar{r}-(\nabla \bar{u})^{T} \bar{r}=-\Delta \bar{r}+\nabla \widetilde{\Pi}+(\nabla \bar{n})^{T} \bar{p}+(\nabla \bar{c})^{T} \bar{q}-\left\|\bar{u}-u^{d}\right\|_{L^{4}}^{4}\left|\bar{u}-u^{d}\right|^{2}\left(\bar{u}-u^{d}\right), \\
\nabla \cdot \bar{r}=0,
\end{array}\right. \\
{\left[\int_{0}^{T} \int_{\Omega}\left(\alpha_{1} \bar{f}+\bar{r}\right)(f-\bar{f}), \int_{0}^{T} \int_{\Omega}\left(\alpha_{2}|\bar{g}|^{2} \bar{g}+\bar{c} \bar{q}\right)(g-\bar{g})\right] \geq[0,0], \quad \forall[f, g] \in \mathcal{U}_{a d} .}
\end{gathered}
$$

Finally, we have the extra regularity $\bar{f}, \bar{g} \in H^{1}\left(\Omega_{T}\right) \cap C\left([0, T], H^{1}\right) \cap L^{2}\left(W^{1,6}\right) \cap L^{2}(C(\bar{\Omega}))$.

Proof. Since $\mathcal{U}_{a d}$ is nonempty, there exists a minimizing sequence $\left\{\left[g_{k}, f_{k}\right]\right\}_{k=1}^{\infty} \subset \mathcal{U}_{a d}$ of (P). Let $\left[n_{k}, c_{k}, u_{k}\right]=$ $G\left(\left[g_{k}, f_{k}\right]\right) \in \mathcal{X}_{4} \times \mathcal{X}_{4} \times \mathcal{X}_{2}$, according to Corollary 4.5. From the definition of the functional $J$,

$$
\begin{aligned}
\frac{7}{20}\left\|n_{k}\right\|_{L^{20 / 7}\left(L^{20 / 7}\right)}^{20 / 7}+\frac{1}{2}\left\|c_{k}\right\|_{L^{2}\left(L^{2}\right)}^{2}+\frac{1}{8}\left\|u_{k}\right\|_{L^{8}\left(L^{4}\right)}^{8}+\frac{\alpha_{1}}{2}\left\|f_{k}\right\|_{L^{2}\left(L^{2}\right)}^{2}+\frac{\alpha_{2}}{4}\left\|g_{k}\right\|_{L^{4}\left(L^{4}\right)}^{4} & \\
& \leq J\left(\left[g_{k}, f_{k}\right]\right) \leq J\left(\left[g_{1}, f_{1}\right]\right)<\infty, \forall k .
\end{aligned}
$$

Hence, $\left\{\left[g_{k}, f_{k}\right]\right\}_{k=1}^{\infty}$ and $\left\{\left[n_{k}, c_{k}, u_{k}\right]\right\}_{k=1}^{\infty}$ are bounded in $L^{4}\left(L^{4}\right) \times L^{2}\left(L^{2}\right)$ and $L^{20 / 7}\left(L^{20 / 7}\right) \times L^{2}\left(L^{2}\right) \times$ $L^{8}\left(L^{4}\right)$ respectively. Since $\mathcal{F}$ is a closed convex subset of $L^{4}\left(L^{4}\right) \times L^{2}\left(L^{2}\right)$ (hence is weakly closed), there exist subsequences, still denoted by $\left\{\left[g_{k}, f_{k}\right]\right\}_{k=1}^{\infty},\left\{\left[n_{k}, c_{k}, u_{k}\right]\right\}_{k=1}^{\infty}$, such that $\left[g_{k}, f_{k}\right] \rightarrow[\bar{g}, \bar{f}]$ and $\left[n_{k}, c_{k}, u_{k}\right] \rightarrow$ $[\bar{n}, \bar{c}, \bar{u}]$ weakly in $L^{4}\left(L^{4}\right) \times L^{2}\left(L^{2}\right)$ and $L^{4}\left(L^{4}\right) \times L^{2}\left(L^{2}\right) \times L^{8}\left(L^{4}\right)$ respectively. From Theorem 4.2 , we conclude that $\left[n_{k}, c_{k}, u_{k}\right] \rightarrow[\bar{n}, \bar{c}, \bar{u}]$ weakly in $\mathcal{X}_{4} \times \mathcal{X}_{4} \times \mathcal{X}_{2}$.

Then, the following convergences hold, as $k \rightarrow \infty$ :

$$
\begin{aligned}
n_{k} & \rightarrow \bar{n} \text { weakly in } L^{4}\left(W^{2,4}\right) \text { and weakly* in } L^{\infty}\left(W^{3 / 2,4}\right), \\
c_{k} & \rightarrow \bar{c} \text { weakly in } L^{4}\left(W^{2,4}\right) \text { and weakly* in } L^{\infty}\left(W^{3 / 2,4}\right), \\
u_{k} & \rightarrow \bar{u} \text { weakly in } L^{2}\left(H^{2}\right) \text { and weakly* in } L^{\infty}\left(H^{1}\right), \\
\partial_{t} n_{k} & \rightarrow \partial_{t} \bar{n} \text { weakly in } L^{4}\left(L^{4}\right), \\
\partial_{t} c_{k} & \rightarrow \partial_{t} \bar{c} \text { weakly in } L^{4}\left(L^{4}\right), \\
\partial_{t} u_{k} & \rightarrow \partial_{t} \bar{u} \text { weakly in } L^{2}\left(L^{2}\right) .
\end{aligned}
$$

From (5.14)-(5.19), the Aubin-Lions lemma (see [33]), Sobolev embeddings, and the compactness of the embedding $\mathcal{X}_{2} \subset L^{8}\left(L^{4}\right)$, we have

$$
\left[n_{k}, c_{k}, u_{k}\right] \rightarrow[\bar{n}, \bar{c}, \bar{u}] \text { strongly in }\left(C\left([0, T] ; L^{q}\right) \cap L^{4}\left(W^{1, q}\right)\right)^{2} \times L^{8}\left(L^{4}\right), \forall q<\infty .
$$


Moreover, from (5.20) we have that $\left[n_{k}(0), c_{k}(0), u_{k}(0)\right]$ converges to $[\bar{n}(0), \bar{c}(0), \bar{u}(0)]$ in $L^{q} \times L^{q} \times L^{4}$, and since $n_{k}(0)=n_{0}, c_{k}(0)=c_{0}, u_{k}(0)=u_{0}$, we deduce that $\bar{n}(0)=n_{0}, \bar{c}(0)=c_{0}$, and $\bar{u}(0)=u_{0}$. Therefore, we can pass to the limit in (1.2) satisfied by $\left[n_{k}, c_{k}, u_{k}\right]$ as $k$ goes to $+\infty$, and we conclude $[\bar{n}, \bar{c}, \bar{u}]$ is also a solution of (1.2) pointwisely, together with $\widetilde{\pi} \in L^{2}\left(H^{1}\right)$. Thus we have that $[\bar{g}, \bar{f}] \in \mathcal{U}_{a d}$, and

$$
\lim _{k \rightarrow \infty} J\left(\left[g_{k}, f_{k}\right]\right)=\inf _{[g, f] \in \mathcal{U}_{a d}} J([g, f]) \leq J([\bar{g}, \bar{f}]) .
$$

Since $J$ is lower semicontinuous on $\mathcal{U}_{a d}$ we get the result. The optimality system (5.11)-(5.13) can be obtained by using Theorem 5.2 .

\section{Conclusions}

In this paper, we study an optimal control problem associated to a 3D-chemotaxis-Navier-Stokes model. First we prove the existence of global weak solutions of the state equations with a linear reaction term on the chemical concentration equation, and an external source on the velocity equation, both acting as controls on the system. Second, we establish a regularity criterion to get global-in-time strong solutions. Finally, we prove the existence of an optimal solution, and we establish a first-order optimality condition. Some open questions and future work in this direction may be addressed; for example, the derivation of second order necessary and sufficient optimality conditions, the proposal of a numerical scheme to approximate the control problem in three dimensions providing some error estimates for the difference between locally optimal controls and their discrete approximations.

\section{Appendix A}

In order to get some regularity results, frequently we use the following embedding results

Lemma A.1. Let $p, q, p_{1}, p_{2}, q_{1}, q_{2} \geq 1$ verifying

$$
\frac{1}{p}=\frac{1-\theta}{p_{1}}+\frac{\theta}{p_{2}}, \quad \frac{1}{q}=\frac{1-\theta}{q_{1}}+\frac{\theta}{q_{2}}, \text { with } \theta \in[0,1] .
$$

Then, $L^{p_{1}}\left(L^{q_{1}}\right) \cap L^{p_{2}}\left(L^{q_{2}}\right) \hookrightarrow L^{p}\left(L^{q}\right)$.

Remark A.2. As a consequence of the Sobolev embeddings, we have

$$
W^{r, p}(\Omega) \hookrightarrow H^{s}(\Omega), \quad r, s>0,1<p<2, \text { with } s=N\left(\frac{1}{2}-\frac{1}{p}\right)+r .
$$

Lemma A.3. [[31], p.49] Let $p, p_{1}, p_{2} \geq 1$ and $s_{1}, s_{2} \geq 0$ verifying

$$
s=(1-\theta) s_{1}+\theta s_{2}, \quad \frac{1}{p}=\frac{1-\theta}{p_{1}}+\frac{\theta}{p_{2}}, \text { with } \theta \in[0,1] .
$$

Then, $L^{p_{1}}\left(H^{s_{1}}\right) \cap L^{p_{2}}\left(H^{s_{2}}\right) \hookrightarrow L^{p}\left(H^{s}\right)$.

Lemma A.4. Let $p, q, p_{1}, p_{2}, q_{1} \geq 1$ verifying

$$
\frac{1}{q}=\frac{1-\theta}{q_{1}}+\theta\left(\frac{1}{p_{1}}-\frac{r}{N}\right), \quad \frac{1}{p}=\frac{\theta}{p_{2}}, \text { with } r>0 \text { and } \theta \in[0,1] .
$$

Then, $L^{\infty}\left(L^{q_{1}}\right) \cap L^{p_{2}}\left(W^{r, p_{1}}\right) \hookrightarrow L^{p}\left(L^{q}\right)$. 
Lemma A.5. [[20], p. xxx] Let $\Omega \subset \mathbb{R}^{N}$ a bounded Lipschitz domain. Then the following interpolation inequality holds:

$$
\|v\|_{W^{\alpha, r}(\Omega)} \leq\|v\|_{W^{\beta, p}(\Omega)}^{\lambda}\|v\|_{W^{\gamma, q}(\Omega)}^{1-\lambda}, 0 \leq \lambda \leq 1
$$

for $0 \leq \alpha, \beta, \gamma \leq 1,1<p, q, r<\infty, \alpha=\lambda \beta+(1-\lambda) \gamma, \frac{1}{r}=\frac{\lambda}{p}+\frac{1-\lambda}{q}$.

Acknowledgements. The authors would like to thank the anonymous referees for useful remarks and suggestions. The authors also thank F. Guillén-González for fruitful discussions on this work.

\section{REFERENCES}

[1] R. Adams and J. Fournier, Sobolev spaces. Elsevier (2003).

[2] F. Abergel and E. Casas, Some optimal control problems of multistate equations appearing in fluid mechanics. RAIRO Modél. Math. Anal. Numér. 27 (1993) 223-247.

[3] M. Braukhoff, Global (weak) solution of the chemotaxis-Navier-Stokes equations with non-homogeneous boundary conditions and logistic growth. Annales de l'Institut Henri Poincaré (C) Non Linear Analysis 33 (2016) 1329-1352.

[4] X. Cao and J. Lankeit, Global classical small-data solutions for a three-dimensional chemotaxis Navier-Stokes system involving matrix-valued sensitivities. Calc. Var. Partial Differ. Equ. 55 (2016) 107.

[5] E. Casas, An optimal control problem governed by the evolution Navier-Stokes equations. In Optimal control of viscous flows, Frontiers in applied mathematics, edited by S.S. Sritharan. SIAM, Philadelphia (1998).

[6] E. Casas, Analysis of the velocity tracking control problem for the 3D evolutionary Navier-Stokes equations, optimal control of viscous flow. SIAM J. Control Optim. 54 (2016) 99-128.

[7] M.A. Chaplain and G. Lolas, Mathematical modelling of cancer cell invasion of tissue: the role of the urokinase plasminogen activation system. Math. Models Methods Appl. Sci. 15 (2005) 1685-1734.

[8] M.A. Chaplain and A. Stuart, A model mechanism for the chemotactic response of endothelial cells to tumour angiogenesis factor. IMA J. Math. Appl. Med. Biol. 10 (1993) 149-168.

[9] F.W. Chaves-Silva and S. Guerrero, A uniform controllability result for the Keller-Segel system. Asymptot. Anal. 92 (2015) 313-338.

[10] F.W. Chaves-Silva and S. Guerrero, A controllability result for a chemotaxis-fluid model. J. Differ. Equ. 262 (2017) $4863-4905$.

[11] H.J. Choe and B. Lkhagvasuren, An extension criterion for the local in time solution of the chemotaxis Navier-Stokes equations in the critical Besov spaces. Ann. Uni. Ferrara. Sez. VII Sci. Mat. 63 (2017) 277-288.

[12] H.J. Choe and B. Lkhagvasuren, Global existence result for chemotaxis Navier-Stokes equations in the critical Besov spaces. J. Math. Anal. Appl. 446 (2017) 1415-1426.

[13] H.J. Choe, B. Lkhagvasuren and M. Yang, Wellposedness of the Keller-Segel Navier-Stokes equations in the critical Besov spaces. Commun. Pure Appl. Anal. 14 (2015) 2453-2464.

[14] A. De Araujo, P.M. Magalhaes, Existence of solutions and optimal control for a model of tissue invasion by solid tumours. $J$. Math. Anal. Appl. 421 (2015) 842-877.

[15] C. Dombrowski, L. Cisneros, S. Chatkaew, R.E. Goldstein and J.O. Kessler, Self-concentration and large-scale coherence in bacterial dynamics. Phys. Rev. Lett. 93 (2004) 98-103.

[16] R. Duan and Z. Xiang, A note on global existence for the chemotaxis-Stokes model with nonlinear diffusion. Int. Math. Res. Notices 2014 (2012) 1833-1852.

[17] A. Duarte-Rodríguez, L.C.F. Ferreira and E.J. Villamizar-Roa, Global existence for an attraction-repulsion chemotaxis-fluid model with logistic source. Discrete Contin. Dyn. Syst. Ser. B 24 (2019) 423-447.

[18] A. Duarte-Rodríguez, L.C.F. Ferreira and E.J. Villamizar-Roa, Global existence for an attraction-repulsion chemotaxis-fluid system in a framework of Besov-Morrey type. J. Math. Fluid Mech. 22 (2020) Paper No. 63.

[19] A. Duarte-Rodríguez, M.A. Rodríguez-Bellido, D.A. Rueda-Gómez, E.J. Villamizar-Roa, Numerical analysis for a chemotaxisNavier-Stokes system. ESAIM Math. Model. Numer. Anal. 55 (2021) S417-S445.

[20] E. Feireisl and A. Novotný, Singular limits in thermodynamics of viscous fluids. Adv. Math. Fluid Mech. (2009).

[21] L.C.F. Ferreira and M. Postigo, Global well-posedness and asymptotic behavior in Besov-Morrey spaces for chemotaxis-NavierStokes fluids. J. Math. Phys. 60 (2019) 061502.

[22] K.R. Fister and C.M. Mccarthy, Optimal control of a chemotaxis system. Quart. Appl. Math. 61 (2003) $193-211$.

[23] A. Fursikov, Optimal control of distributed systems. Theory and applications. Trans. Math. Monographs 187 (2000).

[24] F. Guillén-González, E. Mallea-Zepeda and M.A. Rodríguez-Bellido, Optimal bilinear control problem related to a chemorepulsion system in 2D domains. ESAIM: COCV 26 (2020) Paper No. 29, 21 pp.

[25] F. Guillén-González, E. Mallea-Zepeda and M.A. Rodríguez-Bellido, A regularity criterion for a 3D chemo-repulsion system and its application to a bilinear optimal control problem. SIAM J. Control Optim. 58 (2020) 1457-1490.

[26] F. Guillén-González, E. Mallea-Zepeda and E.J. Villamizar-Roa, On a bi-dimensional chemo-repulsion model with nonlinear production and a related optimal control problem. Acta Appl. Math. 170 (2020) 963-979. 
[27] P. Grisvard, Elliptic Problems in Nonsmooth Domains. Pitman Advanced Publishing Program, Boston (1985).

[28] S. Ishida, Global existence and boundedness for chemotaxis-Navier-Stokes systems with position-dependent sensitivity in 2D bounded domains. Discr. Continu. Dyn. Syst. A 35 (2015) 3463-3482.

[29] J. Jiang, H. Wu and S. Zheng, Global existence and asymptotic behavior of solutions to a chemotaxis-fluid system on general bounded domains. Asympt. Anal. 92 (2015) 249-258.

[30] H. Kozono, M. Masanari and Y. Sugiyama, Existence and uniqueness theorem on mild solutions to the Keller-Segel system coupled with the Navier-Stokes fluid. J. Funct. Anal. 270 (2016) 1663-1683.

[31] J.L. Lions and E. Magenes, Problèmes aux limites non homogènes et applications. Vol. 1. Travaux et recherches mathématiques, No. 17 Dunod, Paris (1968).

[32] J.L. Lions and E. Magenes, Non-homogeneous boundary value problems and applications. Springer, Berlin (1972).

[33] J.L. Lions, Quelques méthodes de résolution des problemes aux limites non linéaires. Dunod, Paris (1969).

[34] J. Lankeit, Long-term behaviour in a chemotaxis-fluid system with logistic source. Math. Models Methods Appl. Sci. 26 (2016) 2071-2109.

[35] N.V. Mantzaris, S. Webb and H.G. Othmer, Mathematical modeling of tumor-induced angiogenesis. J. Math. Biol. 49 (2004) $111-187$.

[36] E. Mallea-Zepeda, E. Ortega-Torres and E.J. Villamizar-Roa, A boundary control problem for micropolar fluids. J. Optim. Theory Appl. 169 (2016) 349-369.

[37] L. Nirenberg, On elliptic partial differential equations. Ann. Sc. Norm. Super. Pisa Cl. Sci. 3 (1959) $115-162$.

[38] M.H. Protter and H.F. Weinberger, Maximum Principles in Differential Equations. Prentice-Hall, Inc., Englewood Cliffs, N.J. (1967).

[39] M.A. Rodríguez-Bellido, D.A. Rueda-Gómez, and E.J. Villamizar-Roa, On a distributed control problem for a coupled chemotaxis-fluid model. Discrete Contin. Dyn. Syst. B 23 (2018) 557-571.

[40] S. Ryu and A. Yagi, Optimal control of Keller-Segel equations. J. Math. Anal. Appl. 256 (2001) 45-66.

[41] J. Simon, Compact sets in space $L^{p}(0, T ; B)$. Ann. Mat. Pura Appl. 146 (1987) 65-96.

[42] H. Sohr and W. Wahl, Generic solvability of the equations of Navier-Stokes. Hiroshima Math. J. 17 (1987) 613-625.

[43] H. Sohr, The Navier-Stokes Equations. An Elementary Functional Analytic Approach. Birkhäuser, Basel (2001).

[44] Y. Tao and M. Winkler, Eventual smoothness and stabilization of large-data solutions in a three-dimensional chemotaxis system with consumption of chemoattractant. J. Differ. Equ. 252 (2012) 2520-2543.

[45] Y. Tao and M. Winkler, Locally bounded global solutions in a three-dimensional chemotaxis-Stokes system with nonlinear diffusion. Annales de l'Institut Henri Poincare (C) Non Linear Analysis 30 (2013) 157-178.

[46] R. Temam, Vol. 343 of Navier-Stokes equations: theory and numerical analysis. American Mathematical Society (2001).

[47] I. Tuval, L. Cisneros, C. Dombrowski, C.W. Wolgemuth, J.O. Kessler and R.E. Goldstein, Bacterial swimming and oxygen transport near contact lines. Proc. Natl. Acad. Sci. USA 102 (2005) 2277-2282.

[48] R. Tyson, S. Lubkin and J.D. Murray, Model and analysis of chemotactic bacterial patterns in a liquid medium. J. Math. Biol. 38 (1999) 359-375.

[49] M. Winkler, Global large-data solutions in a chemotaxis-(Navier-)Stokes system model-ing cellular swimming in fluid drops. Commun. Partial Differ. Equ. 37 (2012) 319-351.

[50] M. Winkler, Boundedness and large time behavior in a three-dimensional chemotaxis-Stokes system with nonlinear diffusion and general sensitivity. Calc. Variat. Partial Differ. Equ. 54 (2015) 3789-3828.

[51] M. Winkler, Global weak solutions in a three-dimensional chemotaxis-Navier-Stokes system. Annales de l'Institut Henri Poincaré (C) Non Linear Analysis 33 (2016) 1329-1352.

[52] M. Winkler, Stabilization in a two-dimensional chemotaxis-Navier-Stokes system. Arch. Ration. Mech. Anal. 211 (2014) $455-487$.

[53] M. Winkler, How far do chemotaxis-driven forces influence regularity in the Navier-Stokes system? Trans. Am. Math. Soc. 369 (2017) $3067-3125$.

[54] M. Winkler, Global mass-preserving solutions in a two-dimensional chemotaxis-Stokes system with rotational flux components. J. Evol. Equ. 18 (2018) 1267-1289.

[55] D. Woodward, R. Tyson, M. Myerscough, J.D. Murray, E. Budrene and H. Berg, Spatio-temporal patterns generated by Salmonella typhimurium. Biophys. J. 68 (1995) 2181-2189.

[56] Q. Zhang, Local well-posedness for the chemotaxis-Navier-Stokes equations in Besov spaces. Nonlinear Anal.: Real World Appl. 17 (2014) 89-100.

[57] Q. Zhang and Y. Li. Convergence rates of solutions for a two-dimensional chemotaxis-Navier-Stokes system. Discr. Contin. Dynam. Syst. Ser. B 20 (2015) 2751-2759.

[58] Q. Zhang and Y. Li, Global weak solutions for the three-dimensional chemotaxis-Navier-Stokes system with nonlinear diffusion. J. Differ. Equ. 259 (2015) 3730-3754. 